\title{
FABRICATION AND OPTIMIZATION OF THREE-DIMENSIONAL METAMATERIALS FOR TERAHERTZ ENERGY-HARVESTING
}

A Dissertation
presented to
the Faculty of the Graduate School
at the University of Missouri
In Partial Fulfillment
of the Requirements for the Degree
Doctor of Philosophy
SHENDU YANG
Dr. Patrick Pinhero, Dissertation Supervisor
DECEMBER 2018


(C) Copyright by Shendu Yang 2018

All Rights Reserved 
The undersigned, appointed by the dean of the Graduate School, have examined the dissertation entitled

\section{FABRICATION AND OPTIMIZATION OF THREE-DIMENSIONAL METAMATERIALS FOR TERAHERTZ ENERGY-HARVESTING}

presented by Shendu Yang,

a candidate for the degree of Doctor of Philosophy, and hereby certify that, in their opinion, it is worthy of acceptance.

Professor Patrick Pinhero, chair

Professor Bret Ulery, member

Professor Karl Hammond, member

Professor Qingsong Yu, member 
This dissertation is dedicated to my mother. 


\section{ACKNOWLEDGMENTS}

First, I sincerely thank my advisor, Dr. Patrick Pinhero, for all his help, support and remarkable ideas that made this dissertation work successful. I thank Dr. Bret Ulery for mentoring me with leveler synthesis. I thank Dr. Karl Hammond for trou-

bleshooting $\mathrm{AT}_{\mathrm{E} X}$ problems in my writing. I thank Dr. Qingsong Yu for sharing me with the experimental equipment in his laboratory and giving me very useful advice on job search.

I give my special thanks to Dr. Zach Thacker who has been supporting me with helpful suggestions and support throughout the whole research project. And many thanks to Mr. Evan Alison and Mr. Hang Xiao for measurement support. In addition, I thank five undergraduate students, Mr. Sean Noble, Mr. Mathew Rasmussen, Mr. Levi Morris, Ms. Megan Bennett, and Mr. Nicholas Cole, who assisted me with electrodeposition and physical vapor deposition.

Finally, I thank my family, especially my mother Peizi Ma, for their help and encouragement. 


\section{TABLE OF CONTENTS}

ACKNOWLEDGMENTS $\ldots \ldots \ldots \ldots \ldots \ldots \ldots \ldots \ldots$ ii

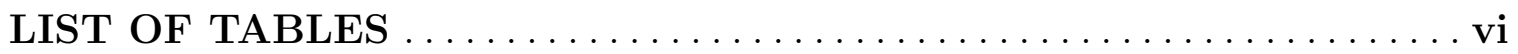

LIST OF FIGURES $\ldots \ldots \ldots \ldots \ldots \ldots \ldots \ldots \ldots \ldots \ldots \ldots \ldots \ldots$ vii

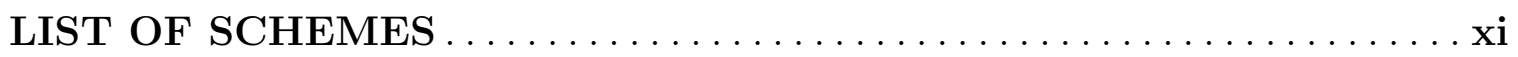

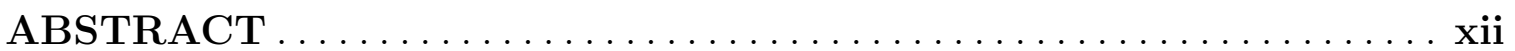

\section{Chapter}

1. INTRODUCTION $\ldots \ldots \ldots \ldots \ldots \ldots \ldots \ldots \ldots \ldots \ldots \ldots \ldots \ldots \ldots \ldots \ldots$

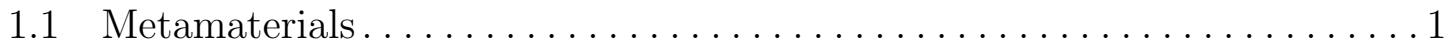

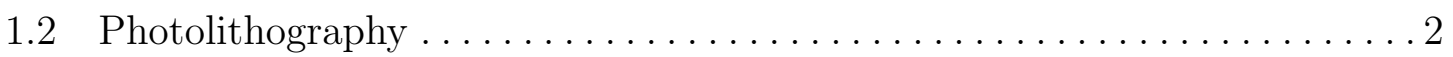

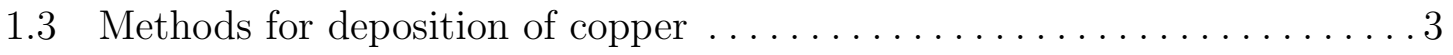

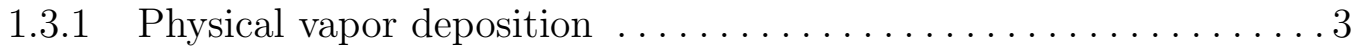

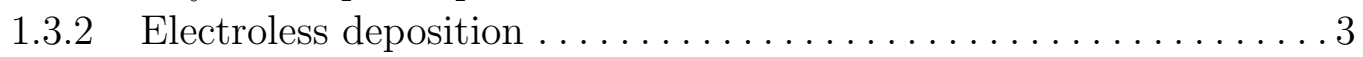

$1.3 .3 \quad$ Electrodeposition.............................. 4

1.4 Additives for electrodeposition of copper .................... 8

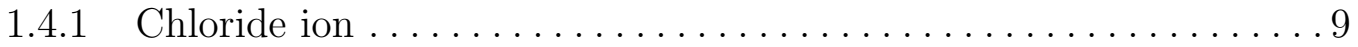

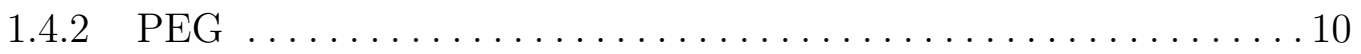

1.4 .3 Accelerator . . . . . . . . . . . . . . . . . . . . . . . . . 10

1.4.4 Leveler ........................................11

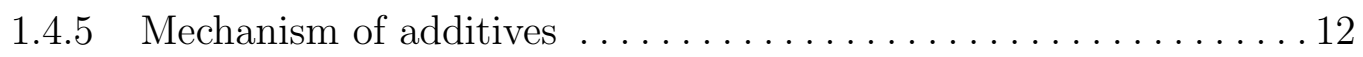

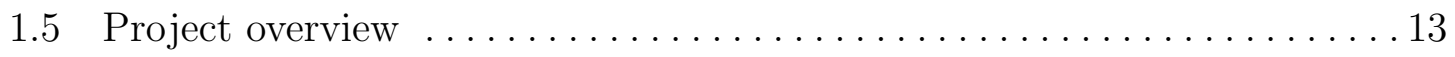

2. FABRICATION OF THREE-DIMENSIONAL METAMATERI-

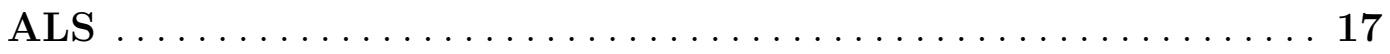

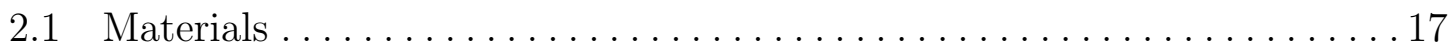

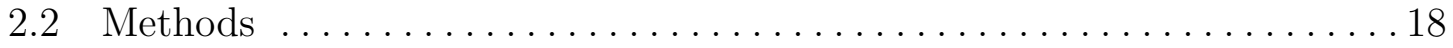


2.2.1 The fabrication of the dielectric part of MMs $\ldots \ldots \ldots \ldots \ldots 18$

2.2.2 The fabrication of the metallic part of MMs . . . . . . . . . . 21

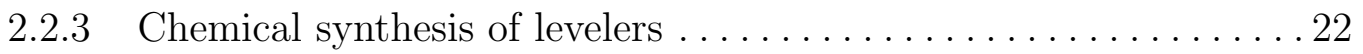

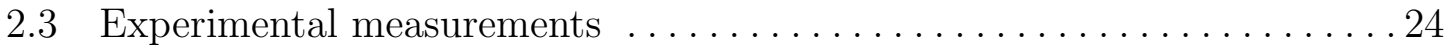

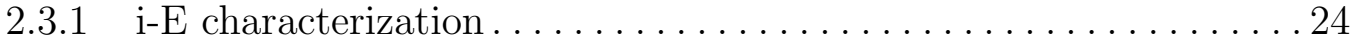

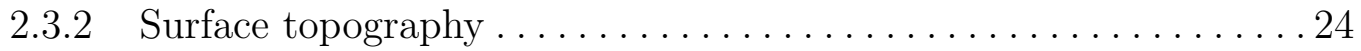

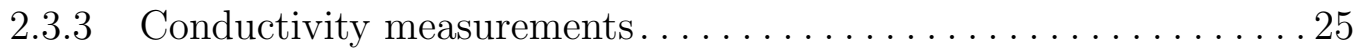

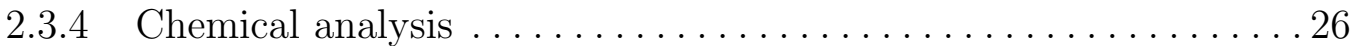

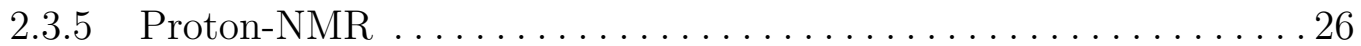

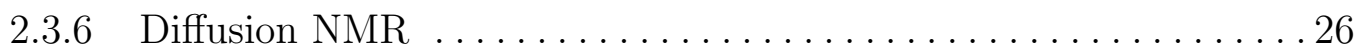

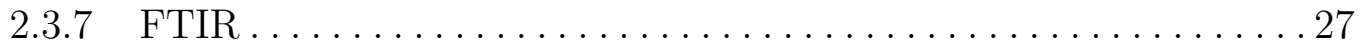

2.3.8 Terahertz time-domain spectroscopy $(\mathrm{THz}-\mathrm{TDS}) \ldots \ldots \ldots \ldots 27$

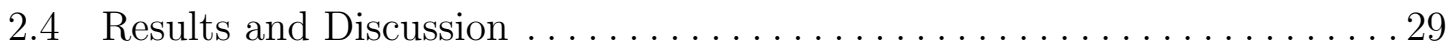

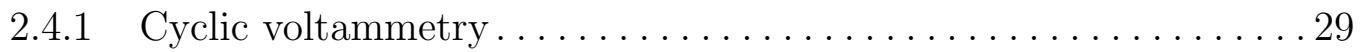

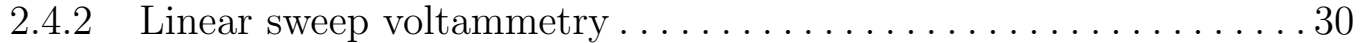

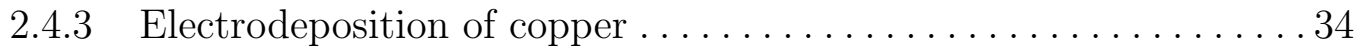

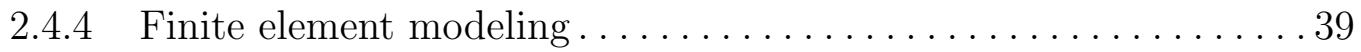

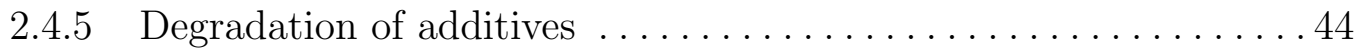

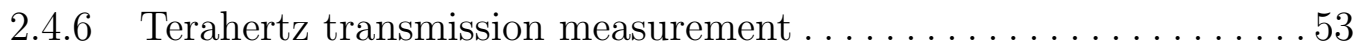

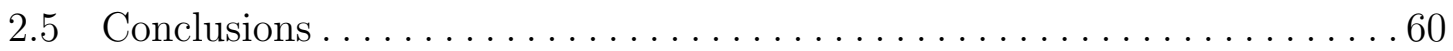

3. OPTIMIZATION OF THREE-DIMENSIONAL METAMATERIAL:

ELECTROMAGNETIC SIMULATION . . . . . . . . . . . 61

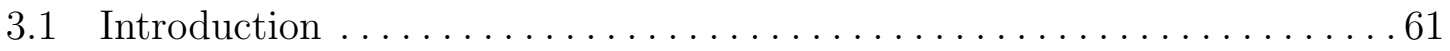

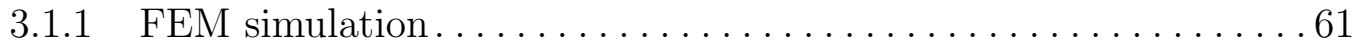

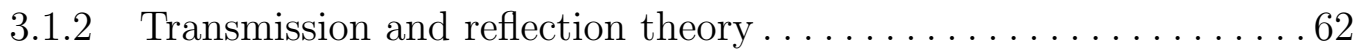

3.1 .3 Drude model . . . . . . . . . . . . . . . . . . . 67

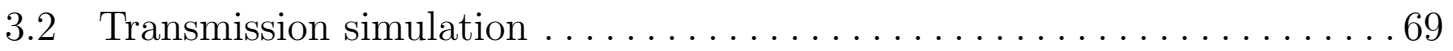

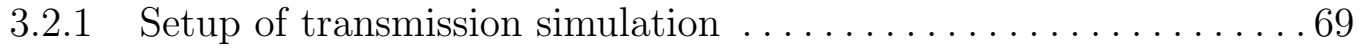

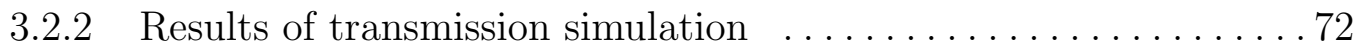

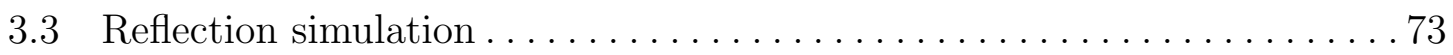

3.3.1 Setup of reflection simulation $\ldots \ldots \ldots \ldots \ldots \ldots \ldots \ldots \ldots \ldots$

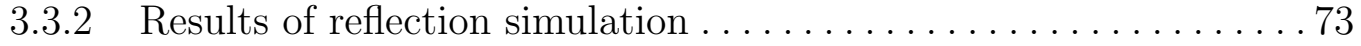

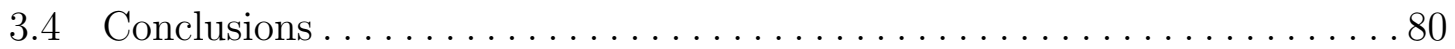


4. SUMMARY AND CONCLUDING REMARKS

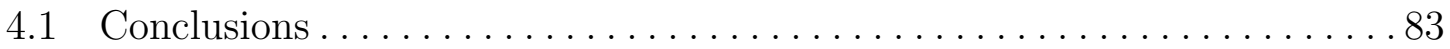

4.1.1 3D THz metamaterial fabrication ................. 83

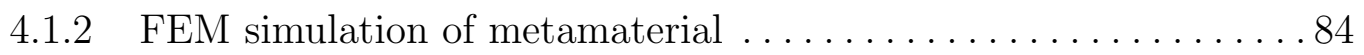

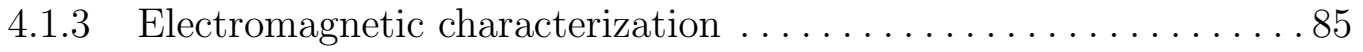

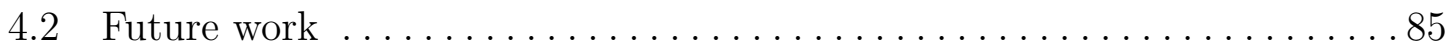

Appendix

A. FOURIER TRANSFORM FOR THZ-TDS $\ldots \ldots \ldots \ldots \ldots \ldots \ldots$

BIBLIOGRAPHY $\ldots \ldots \ldots \ldots \ldots \ldots \ldots \ldots \ldots \ldots \ldots \ldots \ldots$

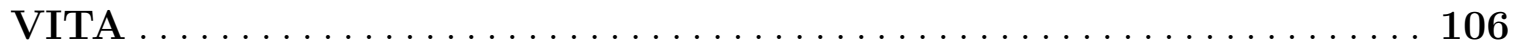




\section{LIST OF TABLES}

Table

Page

2.1 Standard reduction potentials of copper ions in aqueous solution at

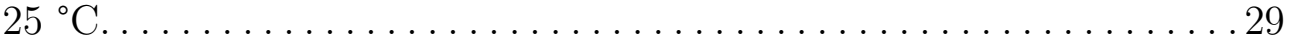

2.2 Analysis summary of the surface topography of copper film that electrodeposited under different potentials in Cl-PEG-PVP-MPSA elec-

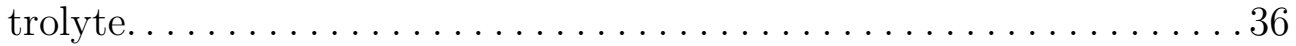

2.3 Parameters used for the COMSOL simulation of superfilling. . . . . . . . 43

3.1 Results of a Drude model fit to the dielectric function of four metals. . . . . 70

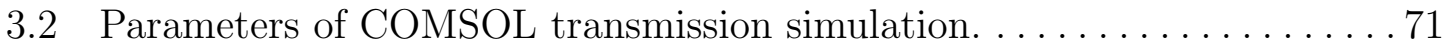

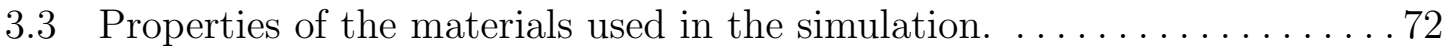




\section{LIST OF FIGURES}

Figure $\quad$ Page

1.1 Schematic of the photolithography process.................... 2

1.2 Overplating height over $0.13 / 0.13 \mu \mathrm{m}$ trench arrays upon $0.7 \mu \mathrm{m}$ field $\mathrm{Cu}$ plating without and with addition of $10 \mathrm{mg} / \mathrm{L}$ PVP into accelerator suppressor electrolyte $\ldots \ldots \ldots \ldots \ldots \ldots \ldots \ldots \ldots \ldots \ldots$

1.3 Chemical structures of the functional groups of levelers: (a) Amine (b) Quaternary ammonium. .........................12

1.4 Mechanism of superfilling and leveling by chloride-carrier-acceleratorleveler additive system for electrodeposition of copper..........12

1.5 Schematic of a MM unit that consists of an SU-8 pillar and a layer of copper..................................... 14

1.6 Schematic of the $1 \mathrm{THz}$ antenna coupled to an MIM diode........... 15

2.1 Top-view images of the substrates containing trenches and pillars....... 18

2.2 Schematic of thermal evaporation. . . . . . . . . . . . . . . . . 19

2.3 Photolithography procedure for the fabrication of MMs. ............ 20

2.4 Schematic of a standard three-electrode cell ...................21

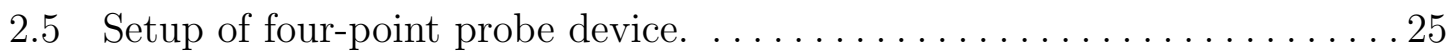

2.6 Schematic of terahertz time-domain spectroscopy. ............... 28

2.7 Setup of terahertz time-domain spectroscopy system. . . . . . . . . . 28

2.8 Cyclic voltammogram of the electrolyte consisting of $0.25 \mathrm{M} \mathrm{CuSO}_{4}$, $1.8 \mathrm{M} \mathrm{H}_{2} \mathrm{SO}_{4}, 0.3 \mathrm{~g} / \mathrm{L}$ PEG, $10^{-5} \mathrm{M}$ MPSA, $10^{-3} \mathrm{M} \mathrm{Cl}^{-}$and 20

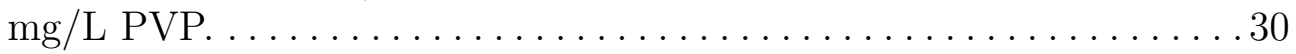


2.9 LSV curves obtained in additive-free electrolyte and electrolyte with $\mathrm{Cl}^{-}$and PEG, respectively. ........................

2.10 LSV curves obtained in acid copper electrolytes with PEG, MPSA and

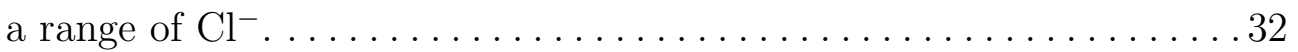

2.11 LSV curves obtained from acid copper electrolytes with and without PEG, respectively. ............................. 32

2.12 LSV curves obtained in acid copper electrolytes with and without $\mathrm{Cl}^{-}$,

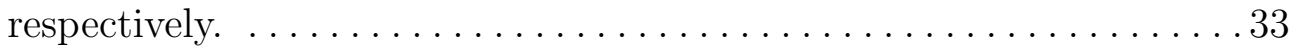

2.13 LSV curves obtained in acid copper electrolytes with Cl-PEG and a

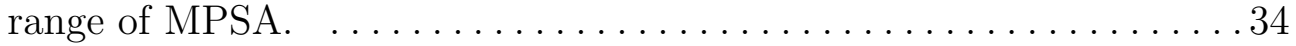

2.14 Comparison of controlled potential electrodepositions in Cl-PEG-PVPMPSA electrolyte under different potentials ranging from -0.04 to -0.25 V. . . . . . . . . . . . . . . . . . . . . . . 35

2.15 Optical microscope images of copper film electrodeposited under different potentials in Cl-PEG-PVP-MPSA electrolyte............. 36

2.16 Optical microscope images of copper film electrodeposited under different potentials in Cl-PEG-PVP-MPSA electrolyte............ 37

2.17 Comparison of the chemical structure and the leveling effect of PVP

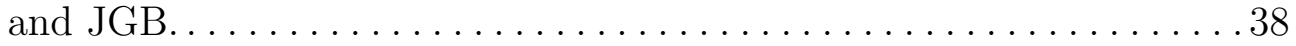

2.18 The initial COMSOL simulation of electrodeposition of copper. ........ 39

2.19 Final COMSOL simulation of electrodeposition of copper............41

2.20 Geometry of the trench for the COMSOL simulation of superfilling. . ... . 42

2.21 Cross-section view of the trenches with different aspect ratios and the corresponding profile and surface concentration of accelerators after one hour of electrodeposition. . . . . . . . . . . . . . . . . 44

2.22 Optical microscope images of the surface topography of $150 \mu \mathrm{m}$-thick copper film deposited in a Cl-PEG-MPSA-PVP additive system of

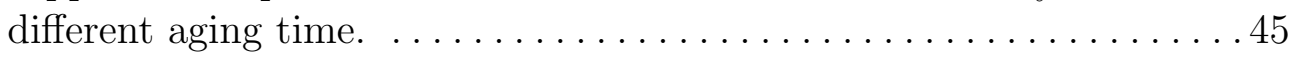

2.23 RMS surface roughness data of the 150 um-thick copper film deposited in Cl-PEG-MPSA-PVP additive system of different aging time. . . . 445 
2.24 Resistivity transients during aging of $150 \mu \mathrm{m}$-thick copper film elec-

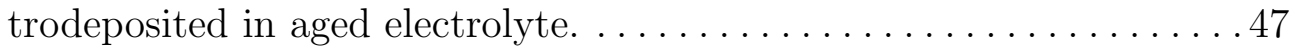

2.25 Cross-section of MMs that electrodeposited in aged electrolyte. . . . . . 44

2.26 Cyclic voltammograms of fresh MPSA and MPSA aged for $1 \mathrm{~h}$ and $6 \mathrm{~h}$

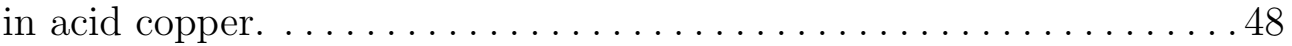

$2.27{ }^{1} \mathrm{H}-\mathrm{NMR}$ spectra of MPSA aged in water and MPSA aged in acid

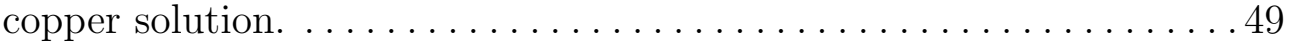

$2.28{ }^{1} \mathrm{H}-\mathrm{NMR}$ spectra and the FTIR spectra of fresh MPSA and the powder dried from MPSA + DI water solution. . . . . . . . . . . . 51

2.29 Cyclic voltammogram of fresh electrolyte and electrolytes used for elec-

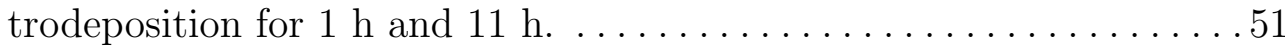

$2.30{ }^{1} \mathrm{H}-\mathrm{NMR}$ spectra of the powder extracted from unused electrolyte and the powder extracted from used electrolyte. . . . . . . . . . . 52

2.31 Terahertz transmission to MMs with 150 um-tall SU-8 pillars and dif-

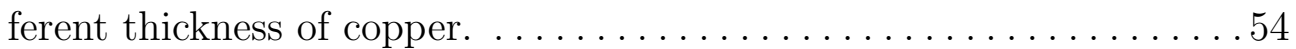

2.32 Terahertz transmission to MMs with $40 \mu \mathrm{m}$-tall SU-8 pillars and dif-

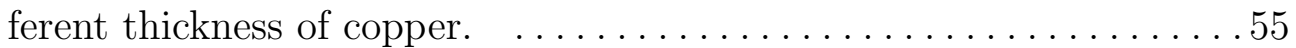

2.33 Comparison of transmission for MMs with overplated copper. . . . . . . 56

2.34 Comparison of transmission for MMs with $100 \mathrm{~nm}$ thick copper and

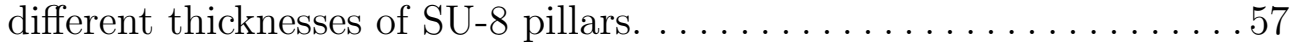

2.35 Comparison of transmission for MMs with halfway-covered copper. . . . .58

2.36 Comparison of transmission for MMs with fully-covered copper. . . . . . . 59

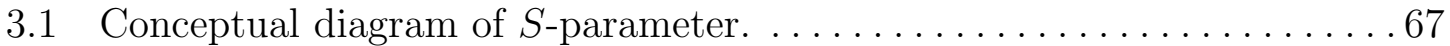

3.2 Dispersion of the real and imaginary parts of the dielectric constant. . . . . 68

3.3 Geometry of a MM unit for COMSOL simulation. $\ldots \ldots \ldots \ldots \ldots \ldots 71$

3.4 Experimental results of transmission of terahertz radiation through MMs from 0.4 to $2 \mathrm{THz}$ with different thicknesses of copper. . . . . . .73 
3.5 Simulation results of the transmission of terahertz radiation through MMs from 0.4 to $2 \mathrm{THz}$ with different thicknesses of copper. ......74

3.6 Geometry of a MM unit for reflection simulations. . . . . . . . . . . . 75

3.7 Reflection of MMs from 0.4 to $2 \mathrm{THz}$ with different metals. .........76

3.8 Reflection of MMs from 0.4 to $2 \mathrm{THz}$ with different air distance $d_{3} \ldots \ldots 76$

3.9 Reflection of MMs from 0.4 to $2 \mathrm{THz}$ with different overplated copper

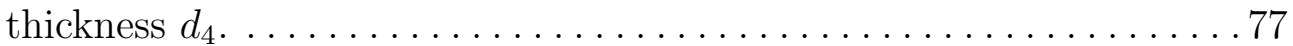

3.10 Reflection of MMs from 0.4 to $2 \mathrm{THz}$ with different SU-8 pillar height d. ........................................... 77

3.11 Reflection of MMs from 0.4 to $2 \mathrm{THz}$ with different SU-8 width $A p . \ldots .78$

3.12 Reflection of MMs from 0.4 to $2 \mathrm{THz}$ with different MM unit spacing

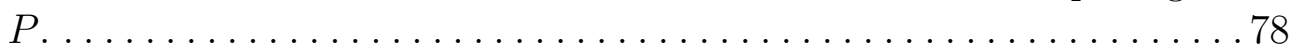

3.13 Final optimized geometry of 3D metamaterial unit. ............... 79

3.14 Reflection of the optimized metamaterial from 0.4 to $2 \mathrm{THz}$......... 80

3.15 Electric field distribution of the optimized metamaterials at different radiation frequencies. . . . . . . . . . . . . . . . . . . . 81 


\section{LIST OF SCHEMES}

Scheme

Page

2.1 Synthetic route for tertiary amine-based polyester, poly (1,8-octanediolco-dipicolinate). Two monomers used in this polymerization reac-

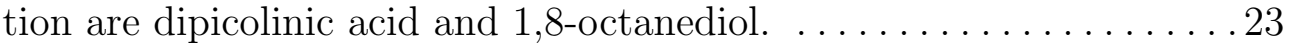

2.2 Synthetic route for tertiary amine-based polyester, poly(triethylene glycol-co-dipicolinate). Two monomers used in this polymerization reaction are dipicolinic acid and triethylene glycol. . . . . . . . 23

4.1 Polymerization reaction of polyanhydride based leveler. . . . . . . . . . 87

4.2 Polymerization reaction of polyamide based leveler. . . . . . . . . . . 87 


\title{
FABRICATION AND OPTIMIZATION OF THREE-DIMENSIONAL METAMATERIALS FOR TERAHERTZ ENERGY-HARVESTING
}

\author{
Shendu Yang
}

Dr. Patrick Pinhero, Dissertation Supervisor

\begin{abstract}
Metamaterials typically consist of metallic and dielectric repeating structures. Electrodeposition of copper is the preferred approach to fabricating the metallic part of the metamaterials of interest in this study. The highly-variant topography requires chemical additives, like chloride ions, 3-mercapto-1-propanesulfonic acid (MPSA), polyethylene glycol (PEG) and polyvinylpyrrolidone (PVP) to enhance bottom-up superfilling while maintaining terrace flatness. This study focuses on both experimental and computational investigations of the degradation potential of the additives and their adsorption mechanism in a highly-acidic copper electrolyte in order to optimally parameterize the copper electrodeposition process. Results show Cl-MPSAPEG-PVP additives perform well, but substitution of PVP with Janus Green B provides better terrace leveling. Additionally, NMR data show a quick and complete conversion of MPSA to bis(3-sulfopropyl) disulfide (SPS) in the acidic copper bath. Finally, FEM simulations further show that the accelerator species may initially accumulate and be transported vertically until overplating, whereby they are transported laterally.

Our study also show that the electromagnetic performance of metamaterial is dependent on SU-8 related parameters, including pillar height, bottom side length and spacing between pillars. The metamaterial geometry is successfully optimized and it can localize the surface plasmon with the peak frequency of $1.2 \mathrm{THz}$ to the nano-antenna and the highest terahertz absorption is $30 \%$.
\end{abstract}




\section{CHAPTER 1}

\section{INTRODUCTION}

\section{$1.1 \quad$ Metamaterials}

Metamaterials typically use metallic structures to provide a negative permittivity and use resonant structures with a scale much smaller than the wavelength of external sources to provide negative permeability leading to a negative refraction [1]. Metamaterial consists of two parts: the dielectric part and the metallic part. Unlike traditional materials whose functionalities are highly related to their composition, the properties of metamaterials are mainly obtained from their repeating structures. Metamaterials are able to manipulate electromagnetic waves and they can be used in many fields such as optical devices, sensors and shielding structures for earthquakes and noise. The ability to tune and switch the properties of materials, something very rarely offered by nature, can be achieved in metamaterials [2]. There is a basic requirement for metamaterial that the size of the repeating structure must be smaller or relative to the wavelength of the external radiation we are interested in.

In 1968, Veselago [3] published a paper discussing what would happen to most natural electromagnetic materials if the permittivity and permeability of the materials were simultaneously negative rather than positive. In 2000, after Smith et al. [4] successfully constructed an artificially structured composite material, electromagnetic metamaterials have been developed rapidly and used as absorbers. In the past decade, metamaterials have been demonstrated in almost every technologically relevant spec-

tral range, from radio [5], microwave [6], milimeter- wave [7], terahertz [8], MIR [9], 


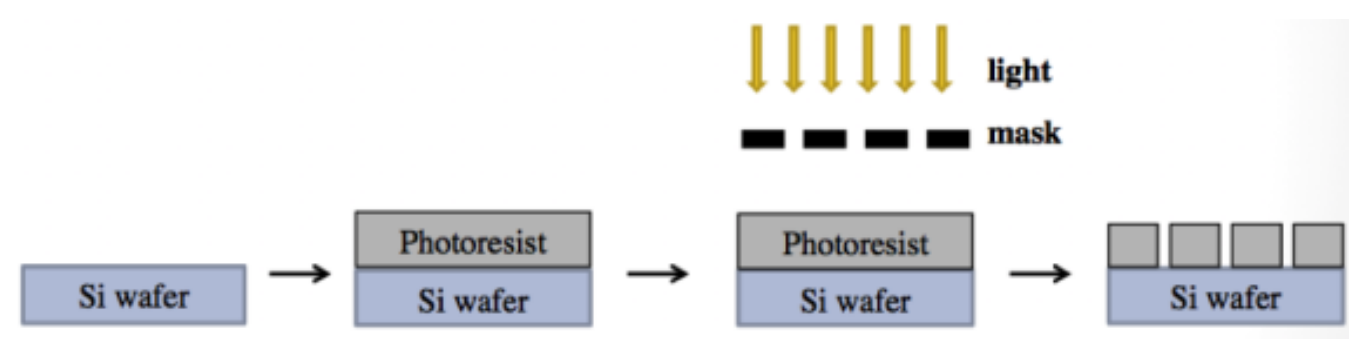

Figure 1.1. Schematic of the photolithography process. A negative photoresist is spincoated on a silicon wafer, after soft and hard baking, the photoresist is hardened. Then it is covered by a photomask and exposed under UV light. The unexposed area can be removed during development.

and NIR [1] to the near optical [10]. Hao et al. designed optical metamaterial absorbers consisting of a layer of gold particles and a gold film, separated by an $\mathrm{Al}_{2} \mathrm{O}_{3}$ dielectric layer, and showed an absorption peak of $88 \%$ is achieved at a wavelength of $1.58 \mu \mathrm{m}[11]$. Metals with a high melting temperature and high conductive coefficient, like $\mathrm{Au}, \mathrm{Cu}$ and $\mathrm{Ni}$, are considered for the metal part of metamaterials [12]. Copper is one of the most common metals in our daily life. It has high conductivity and impressive mechanical properties that are almost as good as silver and gold, making it an excellent alternative for printed wiring boards or for use as electrical wire to conduct electricity because it is much cheaper than gold or silver.

\subsection{Photolithography}

Photolithography is a widely used technique to fabricating semiconductor devices. As shown in Fig. 1.1, photolithography is the process of transferring geometric patterns from a photomask to a light-sensitive chemical often referred to as photoresist that covers a substrate. There are two types of photoresist that have different effects on the substrate: positive and negative. Both photoresists' solubility changes after being exposed to UV light. If the exposed surface becomes more soluble after exposure the photoresist is positive; if it becomes less soluble, the photoresist is negative. After exposing the substrate to UV light, the photoresist needs to be removed 
from the surface using a photoresist developer. In this way, a desired pattern can be created on the substrate's surface.

\subsection{Methods for deposition of copper}

\subsubsection{Physical vapor deposition}

Physical vapor deposition (PVD) processes are atomistic deposition processes in which material is vaporized from a solid or liquid source in the form of atoms or molecules and transported in the form of a vapor through a vacuum or low pressure gaseous (or plasma) environment to the substrate, where it condenses. Typically, PVD processes are used to deposit films with thicknesses in the range of a few nanometers to thousands of nanometers. Typical PVD deposition rates are 1-10 nanometers per second. The main categories of PVD processing are vacuum deposition (evaporation), sputter deposition, arc vapor deposition, and ion plating [13].

\subsubsection{Electroless deposition}

For electroless deposition, there is no power supply providing electrons and the deposition consists of two half reactions, an oxidation reaction, and a reduction reaction. The most common reducing agent is formaldehyde ( $\mathrm{HCHO}$ ) and the overall reaction for electroless copper deposition is:

$$
\mathrm{Cu}^{2+}+2 \mathrm{HCHO}+4 \mathrm{OH}^{-} \longrightarrow \mathrm{Cu}+2 \mathrm{HCOO}^{-}+2 \mathrm{H}_{2} \mathrm{O}+\mathrm{H}_{2} \text {. }
$$

However, it has been found that this bath may release hazardous gases during operation. Therefore, previous papers have investigated electroless copper solutions using non-formaldehyde reducing agents such as dimethylamine borane (DMBA), cobalt(II)-ethylenediamine complex or sodium hypophosphite, which has some advantages such as its low $\mathrm{pH}$, low cost and relative safety. However, unlike the electrodeposition process that can obtain unlimited electrons to continue the reaction, electroless deposition of copper is thickness limited and can only reach $25 \mu \mathrm{m}[14]$. 


\subsubsection{Electrodeposition}

In electrodeposition, it is easy to control the current density and charge during the process of deposition. Several plating systems have been studied such as the alkaline cyanide and pyrophosphate complex ion systems, as well as the acid sulfate and fluoborate simple ion systems. Cyanide solutions are less and less in favor because of their toxicity and are being replaced by non-cyanide solutions. Pyrophosphate solutions, once used heavily for plating through holes on printed wiring boards, have been almost completely replaced by high-throw acid sulfate solutions. Acid copper sulfate solutions are widely used for plating of printed wiring boards and for semicon-

ductor interconnect technology [15]. During an electrodeposition process, a variety of variables come into play such as current density, temperature, composition of the electrolyte, the selection of electrodes, agitation, etc.

\subsubsection{Thermodynamic equilibrium}

For an electrode reaction, equilibrium is characterized by the Nernst equation, which links the electrode potential to the bulk concentration of participants. In the general case:

$$
\mathrm{O}+n \mathrm{e}^{-} \longleftrightarrow \mathrm{R}
$$

where $\mathrm{O}$ and $\mathrm{R}$ are the oxidized and reduced forms, respectively, of the redox couple. Such a reaction will occur in a potential region that makes the electron transfer both thermodynamically and kinetically favorable. The thermodynamic relationship is given by the Nernst equation,

$$
E=E^{\circ}+\frac{R T}{n \mathcal{F}} \ln \frac{a_{O}}{a_{R}}
$$

where $E^{\circ}$ is the standard electric potential, $T$ is the absolute temperature, $R$ is the universal gas constant $(8.314 \mathrm{~J} / \mathrm{K}$. mol. $), n$ is the number of electrons transferred in 
the reaction, $\mathcal{F}$ is the Faraday constant $(96,487 \mathrm{C} / \mathrm{mol}), a_{x}$ is the activity of species $x$.

Since the activity for a solute $x$ is:

$$
a_{x}=\gamma_{x}\left(C_{x} / C^{0}\right)
$$

where $C_{x}$ is the concentration of the solute, $C^{0}$ is the standard concentration (usually $1 \mathrm{M}), \gamma_{x}$ is the activity coefficient of species $x$. Thus, Eqn. 1.1 becomes:

$$
E=E^{\circ}+\frac{R T}{n \mathcal{F}} \ln \frac{\gamma_{O} C_{O}}{\gamma_{R} C_{R}}
$$

In this study, electrodeposition of copper is a process of bulk electrolysis with large currents (above $10 \mathrm{~mA} / \mathrm{cm}^{2}$ ) and experimental time scales on the order of minutes and hours; the basic principles governing electrode reactions still apply. This research applies to the model of deposition of a solid since the reduced species is not soluble in solution. For the reaction

$$
\mathrm{O}+n \mathrm{e}^{-} \longrightarrow \mathrm{R} \text { (solid) }
$$

when more than a monolayer of the reduced species is deposited on an inert electrode (e.g., copper on a platinum electrode) or the deposition is conducted on an electrode made of the same metal as the deposits (e.g., copper on a copper electrode), the activity of $\mathrm{R}, a_{R}$, is constant and equal to unity. Besides, copper is used as an anode that acts as an ion source, and as a result, the concentration of all species remains unchanged. That is, the bulk concentration of solute $x, C_{x}$, is a constant during this process, the same as the initial bulk concentration. In that case, the Nernst equation yields:

$$
E=E^{\circ}+\frac{R T}{n \mathcal{F}} \ln \left[\gamma_{O} C_{i}\right]
$$

where $\gamma_{O}$ is the activity coefficient of species $\mathrm{O}, C_{i}$ is the initial concentration of $\mathrm{O}$. 
If the anode used during electrodeposition is an inert anode such as platinum, the concentration in the solution will change. In this case, the Nernst equation is:

$$
E=E^{\circ}+\frac{R T}{n \mathcal{F}} \ln \left[\gamma_{O} C_{i}(1-x)\right]
$$

where $x$ is the fraction of $\mathrm{O}$ reduced to $\mathrm{R}$ at the electrode potential.

\subsubsection{Calculation of thickness}

For electrodeposition, the most important driving force comes from the external power supply, which can provide large amounts of electrons to the working electrode and maintain the electrodeposition process. The number of electrons is measured in terms of the total charge, $Q$, passed in the circuit. Charge is expressed in units of coulombs (C), where $1 \mathrm{C}$ is equivalent to $6.24 \times 10^{18}$ electrons [16]. The thickness of deposits can be predicted and calculated by Faraday's law:

$$
\frac{\varepsilon \times i}{n \times \mathcal{F}}=\frac{m}{M W \times t}
$$

where $i$ is the observed current, $n$ is the number of electrons, $\mathcal{F}$ is Faraday's constant, $\varepsilon$ is the efficiency, $m$ is the mass of deposits, $M W$ is the molecular weight, and $t$ is the plating time.

\subsubsection{Overpotential deposition}

The overpotential, $\eta$, is the additional potential (beyond the thermodynamic requirement) needed to drive a reaction at a certain rate. Polarization is the departure of the electrode potential (or cell potential) from the equilibrium value upon passage of faradaic current. The extent of polarization is measured by the overpotential [16]:

$$
\eta=E-E_{e q}
$$


The i- $\eta$ curves may be described by the Butler-Volmer equation including cupric ion depletion at high overpotentials:

$$
i=i_{0}\left(1-\frac{i}{i_{L}}\right) \exp \left(-\frac{\alpha \mathcal{F}}{R T}\right) \eta
$$

where overpotential, $\eta$, defines the deviation from equilibrium; the exchange current density, $i_{0}$, describes the dynamic exchange that characterizes equilibrium; the transport-limited deposition rate, $i_{L}$, is determined by boundary layer diffusion and free convection; and the transfer coefficient, $\alpha$, defines the slope of the curve [17].

\subsubsection{Mass transfer of electrodeposition}

Mass transfer in solution occurs by diffusion, migration and convection. In this study, convection can be neglected since there is no stirring or adding electrodes during the electrodeposition of copper. Fick's laws are differential equations describing the flux of a substance and its concentration as functions of time and position. Derived from Fick's first law, Fick's second law pertains to the change in concentration of $\mathrm{O}$ with time [16]:

$$
\frac{\partial C_{O}(x, t)}{\partial t}=D\left(\frac{\partial^{2} C_{O}(x, t)}{\partial x^{2}}\right)
$$

The electrolysis cell is usually large compared to the characteristic length of diffusion; hence, the solution at the walls of the cell is not altered by the process at the electrode, which is equivalent to semi-infinite boundary conditions. For a planar electrode and unstirred solution, the initial and boundary conditions are:

$$
\begin{array}{r}
C_{O}(x, t)=C_{O}^{*} \\
\lim _{x \rightarrow \infty} C_{O}(x, t)=C_{O}^{*} \\
C_{O}(0, t)=0, \quad(t>0)
\end{array}
$$


where $C_{O}^{*}$ is the bulk concentration of $\mathrm{O}$ in the electrolyte. The initial condition represents the homogeneity of the electrolyte before the electrodeposition starts at $t=0$. The second semi-infinite condition is an expression that regions distant from the electrode are unperturbed by the experiment. The last one is the condition at the electrode surface after the potential transition.

\subsubsection{Anode}

For electroplating, the anode is typically made of the same metal as that which is being plated; in this case copper acts as an ion source [18]. However, most copper interconnect plating tools use a phosphorous copper anode containing a small amount of phosphorus ( $0.04 \%$ to $0.06 \%)$. Phosphorous copper anodes are known to behave differently than pure copper anodes in a copper electroplating bath. First, phosphorus is a non-conductor and even a small amount can abruptly decrease the electrical conductivity of the copper anode. Also, the black anodic film produced during the dissolution of phosphorus-containing copper anodes enhances anodic dissolution. In addition, phosphorus promotes direct oxidation of copper metal to $\mathrm{Cu}^{2+}$, counteracting the chloride ion effect and preventing the slime formation associated with the poor adhesion of the $\mathrm{CuCl}$ film formed at the electrode surface [19].

\subsection{Additives for electrodeposition of copper}

Since electroplated copper is widely used in irregularly shaped objects, a big challenge is how to obtain copper deposits of uniform thickness and allow for super-filling of trenches. Moreover, it is hard to control the mass diffusion and local concentration manually during the process of electrodeposition. Therefore, additives are introduced to solve this issue and further refine the deposit characteristics. Organic additives are employed to improve grain refinement, throwing power, leveling and brightening of the deposit [18]. Chloride ions and another three classes of organic compounds called carriers, brighteners and levelers have been used as additives for the electrodeposition 
of copper. Polyalkylene glycol is used as a carrier owing to its large molecular weight, molecules with thiol and sulfonic acid are used as brighteners, while molecules having an amine or quaternary ammonium functional group are used as levelers [20].

The first description of the mechanism of additives was adopted on the theory of additive-induced leveling, whereby additives adsorb at the surface of the substrate, thereby inhibiting the metal reduction process. Only a small amount of additives are added into the electrolyte so the diffusion of additives is limited. In order to solve the transport problem for the deposition process, additives must either be incorporated in the growing film or undergo reductive desorption during metal deposition [21]. The local concentration of additives on the bottoms is lower than on the top of the gaps and trenches so a model must be built up so that the deposition occurs at a lower concentration first and then a higher concentration.

\subsubsection{Chloride ion}

As a kind of common anion, the chloride ion is important for superconformal electrodeposition of copper, and the character of copper deposits are highly influenced by additives. Small amounts of chloride ion have an accelerating effect on the electrodeposition of copper because they act as a linking agent for the carrier and copper ion to reduce anode polarization and help to refine the deposit morphology. When consumable (copper) anodes are used, chloride ions aid anode corrosion, setting up a uniform and adherent anode film [18]. Tan et al. [22] reported that in the absence of chloride ions, accelerators added to the plating solution inhibited copper deposition. Upon the addition of $\mathrm{Cl}^{-}$, a rapid transition from inhibition to acceleration was observed for accelerators, illustrating the critical role played by the chloride ion in these systems. It is important to maintain the concentration of chloride ions at 10 to $80 \mathrm{ppm}$. Below or above that range gives rise to bad deposits of copper [15]. 
Furthermore, the chloride concentration must be kept below $1 \mathrm{mM}$ in order to avoid precipitation of the $\mathrm{CuCl}$ salt.

\subsubsection{PEG}

Polyethylene glycol (PEG) is a polyether compound with the chemical structure $\mathrm{H}\left(\mathrm{OCH}_{2} \mathrm{CH}_{2}\right)_{n} \mathrm{OH}$. PEGs are prepared by polymerization of ethylene oxide and are commercially available over a wide range of molecular weights from $300 \mathrm{~g} / \mathrm{mol}$ to 10,000,000 g/mol. PEGs are adsorbed at the surface of the cathode and form a monolayer to inhibit the fast and disordered electrodeposition of metal. Since most of the metal surface is not very hydrophilic, as a common wetting agent, PEG is able to wet the cathode surface thoroughly and make sure the cathode can have good contact with the electrolyte.

\subsubsection{Accelerator}

MPSA, or 3-mercaptopropanesulfonic acid sodium salt, is a kind of additive with an accelerating effect for acid copper deposition. Some studies have examined the influence of the accelerator and its role in bottom-up deposition. According to Tan et al. [22], electrodeposition from acidic copper electrolytes containing MPSA was inhibited due to a strongly adsorbed surface layer that was dependent on the concentration of SPS/MPSA, however, the inhibition was readily eliminated upon the addition of chloride and resulted in an overall acceleration. A study proposed by Moffat et al. [21] showed that superfilling of 90-500 $\mathrm{nm}$ trenches can be obtained by Cl-PEG-MPSA electrolyte. Among all the additives, MPSA is the most unstable because thiols $(\mathrm{S}-\mathrm{H})$ would be oxidized and form disulfide bonds $(\mathrm{S}-\mathrm{S})$ in the presence of $\mathrm{Cu}^{2+}[23]$ :

$$
2 \mathrm{MPSA}+\mathrm{Cu}^{2+} \longrightarrow \mathrm{SPS}+2 \mathrm{Cu}^{+}+2 \mathrm{H}^{+}
$$



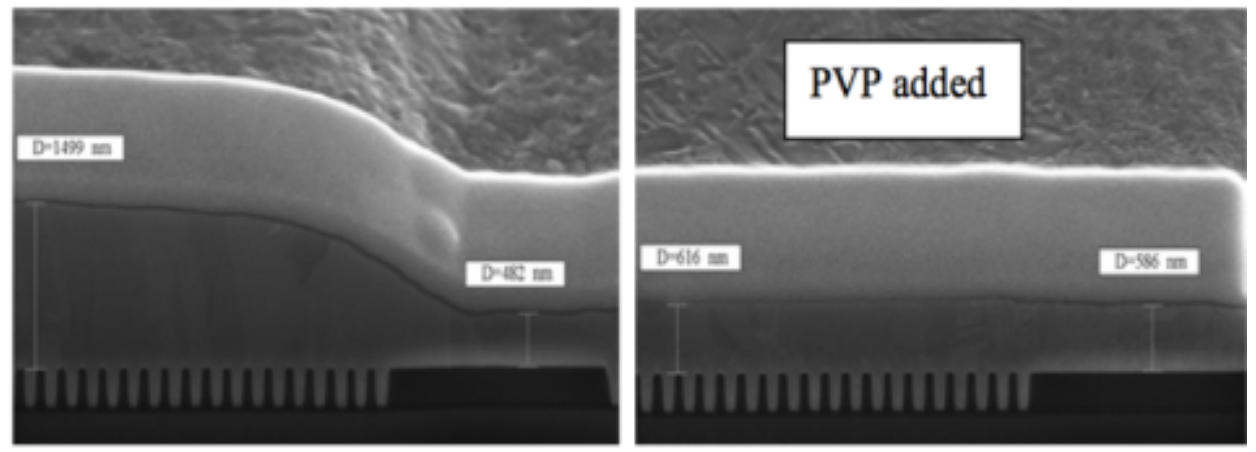

Figure 1.2. Overplating height over $0.13 / 0.13 \mu \mathrm{m}$ trench arrays upon $0.7 \mu \mathrm{m}$ field $\mathrm{Cu}$ plating without and with addition of $10 \mathrm{mg} / \mathrm{L} \mathrm{PVP}$ into accelerator suppressor electrolyte [27].

\subsubsection{Leveler}

Levelers is a type of nitrogen-compounds that preferentionly adsorb on the peaks of a substrate and inhibit electrodeposition on those area. Levelers are typically molecules having quaternary ammonium cations such as Janus Green B (JGB) [24] and Diazine Black (DB) [25], or having amine functionality, such as polyvinylpyrrolidone (PVP) [26]. As shown in Fig. 1.2, leveler PVP can rapidly reduce overplating above the superfilled area. Other research studied the impact of the leveler molecular weight and concentration on copper electroplating for $1 \mu$ m-deep trenches in Cl-SPS-PEG-PVP additive systems and indicated that the best leveling effect can be obtained when the leveler molecular weight is between 29,000 and 55,000 g/mol, the optimal concentration of PVP is $10 \mathrm{ppm}$ and the leveler would not strongly impact bottom-up fill [27].

The chemical structures of the functional groups of levelers are shown in Fig. 1.3. For the levelers with amine functionality, the adsorption of amine onto the copper substrate takes place through the donation of lone pair electrons from the amine to the unoccupied $s$ band of copper [28]. For the levelers with quaternary ammonium cation, they can preferentially adsorb on the the high current density areas and balance the negative charge by electrostatic attraction, inhibiting the electrodeposition on the 


\section{Functional groups of levelers:}

(a) Amine

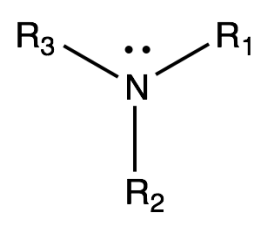

(b) Quaternary ammonium

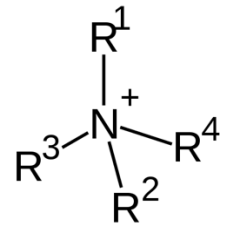

Figure 1.3. Chemical structures of the functional groups of levelers: (a) Amine (b) Quaternary ammonium.

\section{Electric Field}
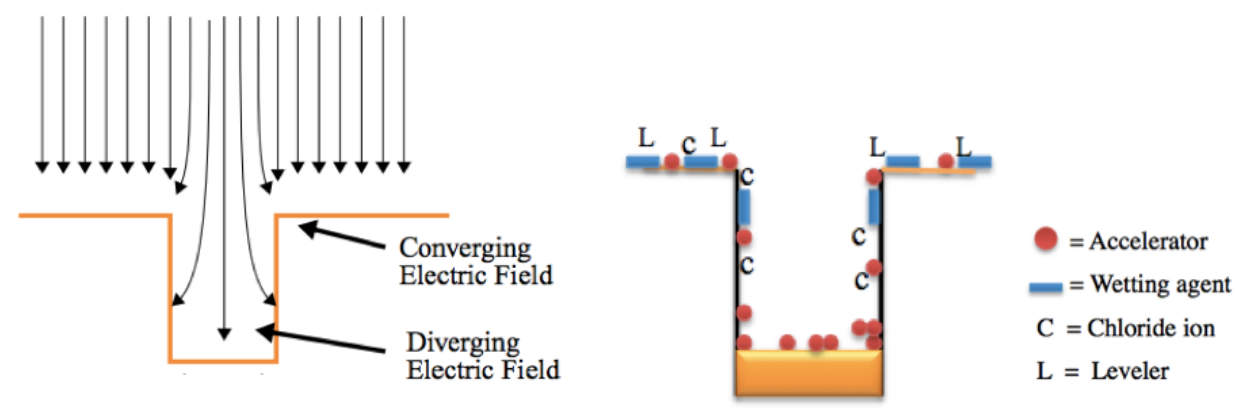

Figure 1.4. Mechanism of superfilling and leveling by chloride-carrier-accelerator-leveler additive system for electrodeposition of copper.

high current density areas (i.e., the convex areas). Since quaternary ammonium cation is a non-metallic cation with permanent charge, it would not be reduced on the substrate during adsorption process. Based on its steady adsorption property, other researchers also used it as a protection layer for anti-corrosion study [29].

\subsubsection{Mechanism of additives}

In our study, the surface of the samples to be electroplated have tall structures that affect the natural distribution of the electric field. Without additives, the electric field is expected to converge at the top of these structures and diverge at the bottom, as shown in Fig. 1.4. As the substrate is immersed into the electrolyte, PEG adsorbs at 
the surface, forming a monolayer to inhibit the fast and disordered electrodeposition of metal. Because most of the metal surface is hydrophobic, PEG is able to wet the cathode surface thoroughly ensuring good contact with the electrolyte [30,31]. The anion of the accelerator additive, MPSA, preferentially locates at areas with the lowest natural current density. In the current study, these areas are the sections of the samples with concave shaped features. As an anion, the accelerator forms a complex with the copper ion, which effectively brings the copper to the lower sections of the features. After electron exchange, copper can be electrodeposited onto the bottom first, causing superfilling of copper to these concave areas.

However, overplating above the superfilled area caused by the accumulation of accelerators is commonly observed. To mitigate this effect, a leveler additive is used. The leveler preferentially adsorbs onto areas with highest current density through amine or quaternary ammonium functionalities, effectively inhibiting deposition and maintaining terrace flatness.

\subsection{Project overview}

Metamaterials (MMs), as a class of artificial material, possess a physical property to manipulate electromagnetic waves by absorbing, bending, or supressing waves due to their repeating sub-wavelength features. In this study, electrodeposition of $\mathrm{Cu}$ was utilized to create MMs and investigated for their potential in an energy harvesting device. This device, seen in Fig. 1.5, consists of a MM coupled to a rectification nano-antenna (rectenna). When an electromagnetic wave, in the form of heat or light, irradiates a metamaterial with a resonant frequency, an intense "plasma" of lower-frequency surface plasmons are generated at the metal/dielectric interface. Since surface plasmons are a type of free electron oscillation, their waves still exhibit electromagnetic characteristics. The purpose of the MM is to convert thermal energy into surface plasmons that are absorbed and rectified by a rectenna [32]. Finally 


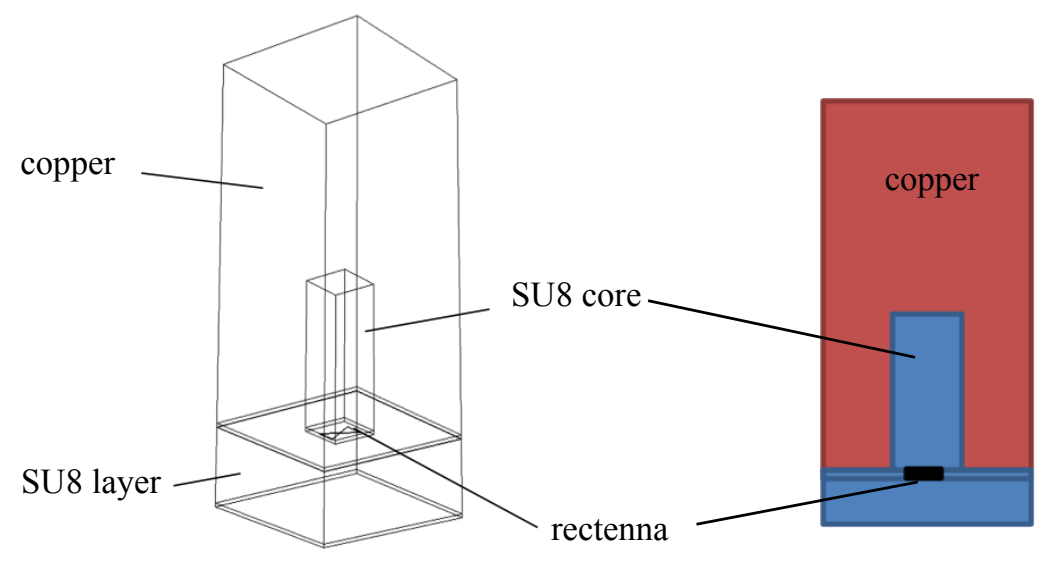

Figure 1.5. Schematic of a MM unit that consists of an SU-8 pillar and a layer of copper. Each MM unit is coupled to a rectenna. The MM unit converts thermal radiation into localized coherent surface plasmons that are subsequently absorbed by each nano-antenna. The antennas focus the electric field to the feed point containing a metal-insulator-metal diode that rectifies the current from $\mathrm{AC}$ to $\mathrm{DC}$.

the electric field is focused to a metal-insulator-metal (MIM) diode that rectifies the current from AC to DC via electron tunneling effect. As shown in Fig. 1.6, a bow-tie shaped antenna is coupled with an MIM diode. The diode area was chosen to be $160,000 \mathrm{~nm}^{2}$ with a thickness of $3 \mathrm{~nm}$.

The spatial dimensions of each functional unit on the device is fabricated relative to the wavelength of EM radiation to be harvested. Sub-wavelength feature is an easy way to couple light into propagating surface plasmons and generate surface plasmon resonance. The target wavelength of the current device was chosen as a compromise between energy density and diode operating efficiency. The efficiency of the current diode design decreases sharply after $1 \mathrm{THz}$ [33]. Besides, the rectenna efficiency is low at high frequencies while the thermal radiation intensity is low at low frequencies [32]. So in this work, the geometry of the device was optimized for operation at $1 \mathrm{THz}$.

In this work, the metamaterial consists of copper and a photoresist, SU-8, in an alternating micron-scale patterns that is fabricated using various techniques, including 


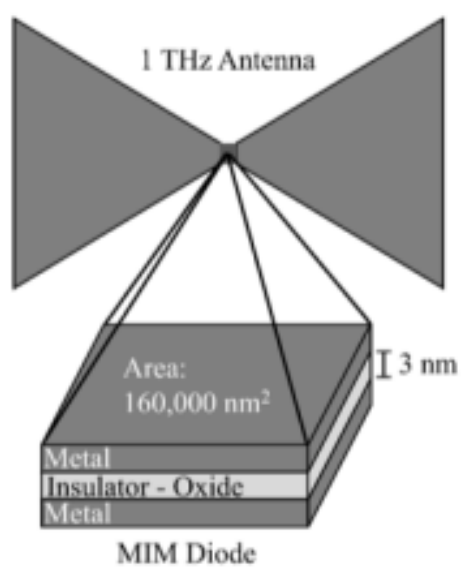

Figure 1.6. Schematic of the $1 \mathrm{THz}$ antenna coupled to an MIM diode.

photolithography, wet and dry etching of metals and oxides, thermal evaporation of thin-layer metals, and electrodeposition of bulk copper. The three-dimensional metamaterials are designed and fabricated to manipulate $0.3-2.0 \mathrm{THz}$ radiation into a well-organized, functional source of plasmons that are rectified by rectenna into a direct current. Each metamaterial unit has a micron-scale SU-8 pillar in the middle and copper all around the pillar. A rectenna is embedded at the base of the pillar where the intensity of the generated electric field is the highest.

In order to align with $1 \mathrm{THz}$ frequency, i.e., $300 \mu \mathrm{m}$ wavelength, $150 \mu \mathrm{m}$-tall SU-8 pillars were designed as the dielectrics part of MMs units. Copper is commonly considered for the metallic part of MMs due to its high melting temperature and high conductive coefficient [12]. In order to deposit at least $150 \mu \mathrm{m}$-thick copper, electrodeposition was used because it has high deposition rate and is widely used in irregular shaped objects. However, a big challenge was to obtain copper deposits of uniform thickness while allowing for super-filling of concave areas and leveling of those that are convex. Moreover, it was difficult to control the mass transfer and local concentration during the process of electrodeposition. Therefore, some additives were used to achieve bottom-up filling, leveling, and brightening of the 
deposit $[18,34,35]$. Chloride ions and another three classes of organic compounds called carriers [36], accelerators [22], and levelers [20,25,37-40] were used as additives for the electrodeposition of copper.

Besides, finite element modeling is employed to simulate the electrodeposition process and the adsorption of additives. In order to get an excellent performance of metamaterial, we optimized the metamaterial structure design via finite element modeling as well. After the desired metamaterial was fabricated, transmission and reflection experiments were carried out with terahertz-time domain spectroscopy to test the electromagnetic performance of the metamaterial. 


\section{CHAPTER 2}

\section{FABRICATION OF THREE-DIMENSIONAL METAMATERIALS}

\subsection{Materials}

Cupric sulfate pentahydrate $\left(\mathrm{CuSO}_{4} \cdot 5 \mathrm{H}_{2} \mathrm{O}\right)$, sulfuric acid $\left(\mathrm{H}_{2} \mathrm{SO}_{4}\right)$, hydrochloric acid $(\mathrm{HCl})$ and sodium 3-mercapto-1-propanesulfonate (MPSA, $\mathrm{NaSO}_{3}\left(\mathrm{CH}_{2}\right)_{3} \mathrm{SH}$ ) were purchased from Fisher Scientific. Polyethylene glycol (PEG, $\mathrm{H}\left(\mathrm{OCH}_{2} \mathrm{CH}_{2}\right)_{n} \mathrm{OH}$, MW: 6000), polyvinylpyrrolidone (PVP, $\left(\mathrm{C}_{6} \mathrm{H}_{9} \mathrm{NO}\right)_{n}$, average MW: 40,000) and Janus Green B (JGB, $\mathrm{C}_{30} \mathrm{H}_{31} \mathrm{CIN}_{6}$ ) were purchased from Alfa Aesar. All the experiments were completed using DI water $(18.2 \mathrm{M} \Omega)$. In our study, the basic bath for the electrolyte consists of $0.25 \mathrm{M} \mathrm{CuSO}_{4}$ and $1.8 \mathrm{M} \mathrm{H}_{2} \mathrm{SO}_{4}$; the additives used are $\mathrm{HCl}$, MPSA, PEG, PVP and JGB.

Other materials and instruments used include copper tape (purchased from Ted Pella), ultrasonic cleaner (Fisher Scientific 220H), acetone, anode bag (purchased from Kocour), nail polish, thermal evaporator (Edwards auto-306), dummy cell, Gamry PCI4/300 potentiostat, diamond wheel saw (South Bay Technology 650), diamond suspension (purchased from South Bay Technology), and release agent/epoxy resin/epoxy hardener/samplkups (purchased from Buehler). Two different kinds of substrates were used as working electrodes for the electrochemical experiment: one is $\mathrm{Si} / \mathrm{SiO}_{2}$ wafers and the other is metamaterial substrates containing either $\mathrm{SU}-8$ trenches or pillars as shown in Fig. 2.1. The $\mathrm{Ag} / \mathrm{AgCl}$ electrode was used as a refer- 

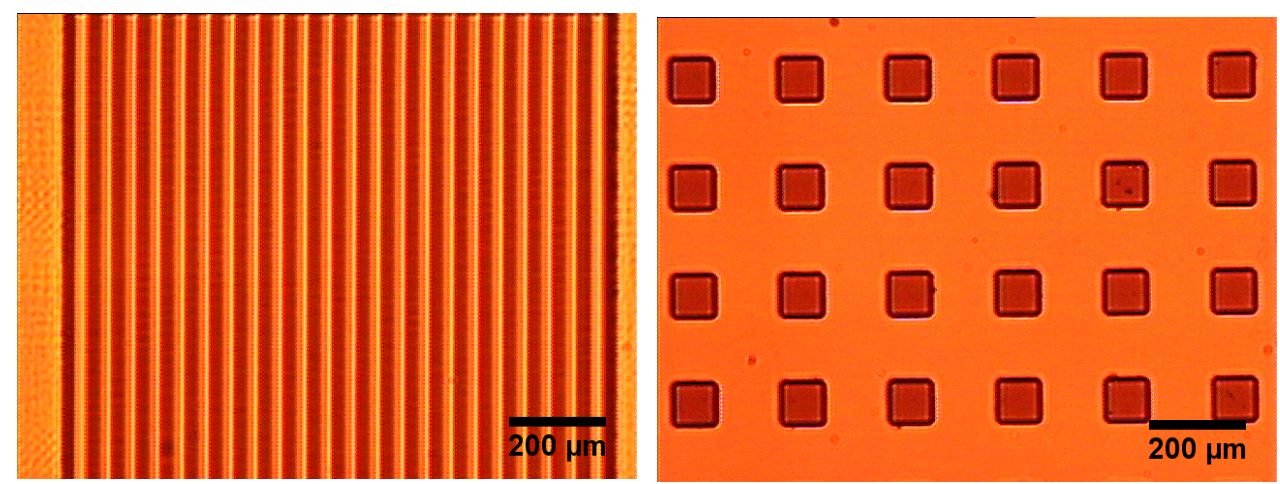

Figure 2.1. Top-view images of the substrates containing trenches and pillars.

ence electrode for all of the measurements. A phosphorous copper anode (purchased from Kocour) was used as counter electrode.

\subsection{Methods}

\subsubsection{The fabrication of the dielectric part of MMs}

Photolithography is used to fabricate the dielectric part of metamaterials. The whole procedure is shown in Fig. 2.3. A basic requirement for working electrodes is that they must be conductive, but the silicon substrate is just a semiconductor so the a thermal evaporator was used to deposit a metal seed layer on the substrates as shown in Fig. 2.2. Before doing thermal evaporation, an $n$-type $\operatorname{Si}(100)$ wafer was first cleaned by plasma ashing with the power of $50 \mathrm{~W}$, under 150 mTorr oxygen for 1 min. Then the silicon wafer was mounted on the holder by screws and tiny clips and the desired face was placed downward in the chamber of the thermal evaporator. A small chunk of metal was placed onto the metallic boat in the chamber. Air was vacuumed out first and the boat was heated up when the pressure in the chamber was around $10^{-5}$ Torr. A detector close to the substrate can detect the thickness of the metal film. The deposition rate is controlled to around 0.1 to $0.2 \mathrm{~nm} / \mathrm{s}$. A 


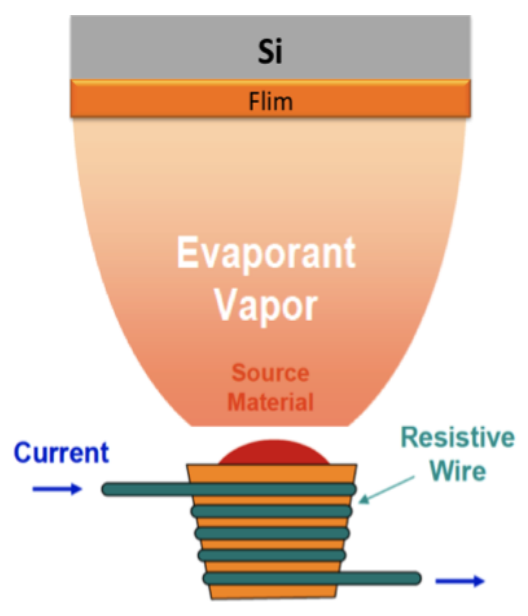

Figure 2.2. Schematic of thermal evaporation. In the chamber of a thermal evaporator, a silicon wafer is mounted upside-down. A copper pellet is placed on the boat below the wafer and the air is pumped out of the chamber until the pressure is around $10^{-5}$ Torr. The copper vapor can be generated when a current is applied to the copper boat, resulting in the deposition of a metal seed layer on the silicon substrate.

$20 \mathrm{~nm}$-thick chrome film was thermo-evaporated onto silicon substrate as an adhesion layer, followed by another 200 nm-thick copper film as a seed layer.

After the wafer was cooled down, a positive photoresist AZ4210 was then spincoated onto the wafer with $500 \mathrm{rpm}$ spread speed for $5 \mathrm{~s}$ and 6000 spin speed for the next $30 \mathrm{~s}$. Then the wafer was soft-baked at $100{ }^{\circ} \mathrm{C}$ for $1 \mathrm{~min}$ and cooled down to room temperature. Since there was a buildup of an edge bead on the outer perimeter of the wafer that causes an uneven attachment between the wafer and the photomask for the following patterning steps, one additional UV exposure under a $100 \mathrm{~mJ} / \mathrm{cm}^{2}$ exposure dose with our customized edge-bead-removal (EBR) mask was carried out. Then the wafer was dipped into AZ developer for $120 \mathrm{~s}$, rinsed with DI water and dried with nitrogen gas. After the edge bead was removed, the EBR mask was replaced with a copper seed layer mask. After the second UV exposure under $40 \mathrm{~mJ} / \mathrm{cm}^{2}$, the wafer was developed and plasma ashed again. The next step was to wet etch the structures. The wafer was dipped into copper etchant 49-1 (ferric chloride) with agitation for 10 to 12 min until the copper pattern was fully etched. A photoresist 


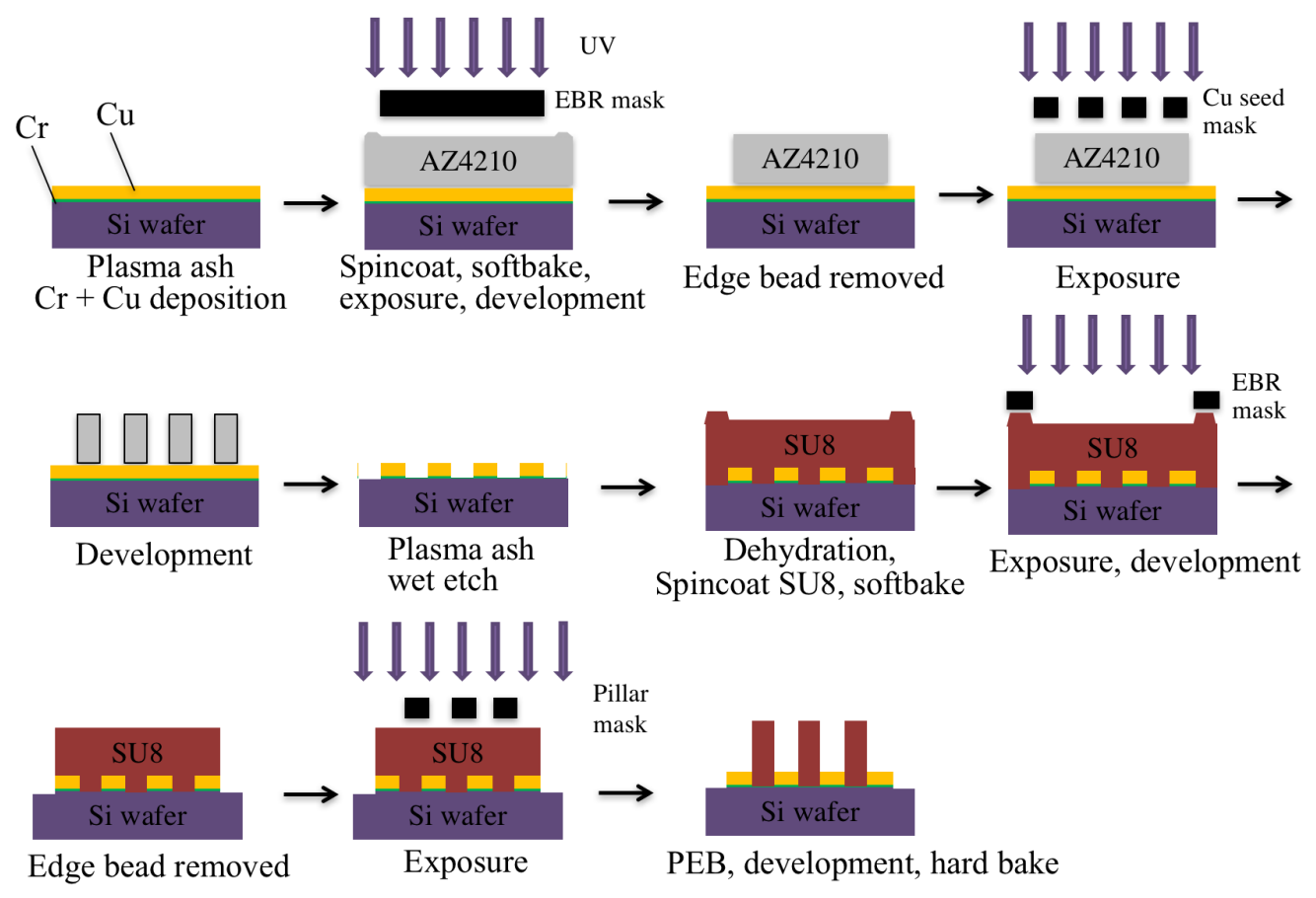

Figure 2.3. Photolithography procedure for the fabrication of MMs.

remover, ( $N$-methylpyrrolidinone), was used for one minute to remove the AZ4210. After spraying it with acetone, IPA and drying it with nitrogen gas, the wafer was dipped into chrome etchant (ceric ammonium nitrate + perchloric acid) for $20 \mathrm{~s}$ until the chrome was removed. After rinsing with DI water and blow drying with nitrogen gas, the desired copper pattern was formed on the wafer. The next step was to fabricate the dielectric features. The wafer was first dehydrated under $150{ }^{\circ} \mathrm{C}$ for 30 min with nitrogen gas purging, then an OmniCoat (adhesion film) was spincoated on the wafer with a spread speed of $500 \mathrm{rpm}$ for $20 \mathrm{~s}$ followed by a $3000 \mathrm{rpm}$ spin speed for $30 \mathrm{~s}$. It was then baked at $150{ }^{\circ} \mathrm{C}$ for $30 \mathrm{~min}$ with nitrogen gas purging. A negative photoresist, SU-8-2035, was warmed up to $40{ }^{\circ} \mathrm{C}$ and poured on the wafer from the center to the edge, then spincoating was carried out with a $500 \mathrm{rpm}$ spread speed for $15 \mathrm{~s}$ and a $2000 \mathrm{rpm}$ spin speed for $30 \mathrm{~s}$. The wafer was then soft-baked from $40{ }^{\circ} \mathrm{C}$ to $60{ }^{\circ} \mathrm{C}$ with a ramp rate of $2{ }^{\circ} \mathrm{C} / \mathrm{min}$ and held for one hour, then the temperature 


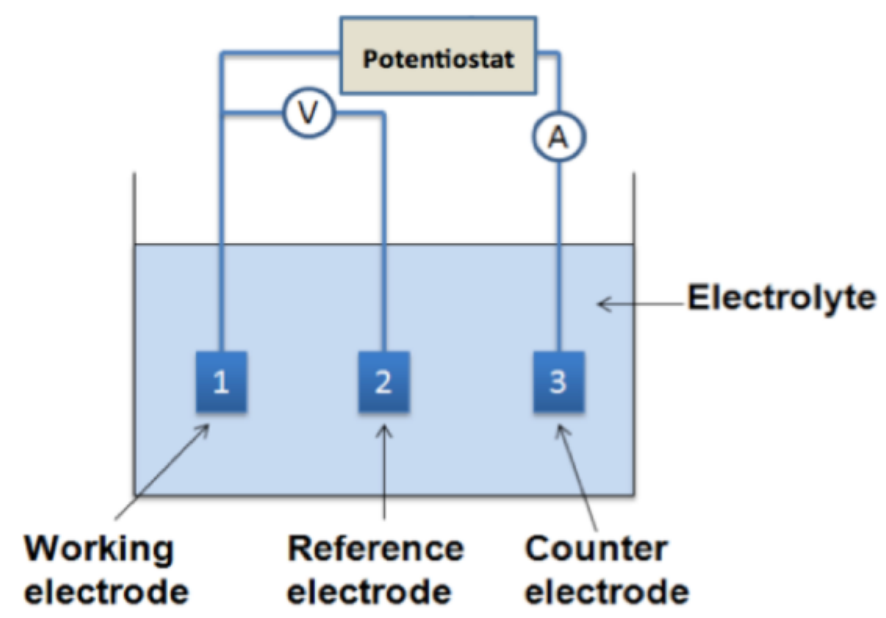

Figure 2.4. Schematic of a standard three-electrode cell that consists of a working electrode, a counter electrode and a reference electrode. All three electrodes are placed into the electrolyte and an external potentiostat is used to control the electrodeposition process.

was increase from $65{ }^{\circ} \mathrm{C}$ to $95{ }^{\circ} \mathrm{C}$ at the same ramp rate and held for two hours. Finally, the wafer was cooled down to room temperature with a ramp rate less than $1{ }^{\circ} \mathrm{C} / \mathrm{min}$. After a thick SU-8 layer was formed, the edge bead of SU-8 was removed by UV exposure. After a final UV exposure with an SU-8 mask, post-exposure-baking (PEB) was carried out from room temperature to $55^{\circ} \mathrm{C}$ with a ramp rate of $1{ }^{\circ} \mathrm{C} / \mathrm{min}$ and a 120 min holding time, then cooled down to room temperature. The wafer was then developed with SU-8 developer for five minutes, sprayed with isopropyl alcohol (IPA) and dried with nitrogen gas. After a final hard bake in a vacuum furnace from room temperature to $150{ }^{\circ} \mathrm{C}$ at a ramp rate of $2{ }^{\circ} \mathrm{C} / \mathrm{min}$, the $3 \mathrm{D} \mathrm{SU}-8$ features were created. As shown in Fig. 2.1, two different SU-8 patterns, trenches and pillars, were fabricated and used in the electrochemical experiments.

\subsubsection{The fabrication of the metallic part of MMs}

All electrochemical experiments were carried out in a conventional three-electrode cell as shown in Fig. 2.4. The metamaterial substrate was masked with finger nail 
polish and only $1 \mathrm{~cm}^{2}$ was exposed as the deposition area. This was done because the interface of the substrate between the electrolyte and the air is likely to become corroded, preventing the deposition from continuing. The top conductive area of the wafer was attached by copper tape in order to connect the alligator clip from the pontentiostat. The electrodeposition was carried out under the mode of controlled potential coulometry or controlled current mode at room temperature. $400 \mathrm{ml}$ of electrolyte was placed in a beaker with a custom-made 3D-printed cover on the top. There were three holes on the cover, with $2 \mathrm{~cm}$ spacing between each hole in case of a big IR drop, also allowing for consistent positioning of the three electrodes for each experiment. No agitation was performed during the experiments. The relationship between charge and the amount of product formed is given by Faraday's law as previously shown in Eqn. 1.6. The total charge can be calculated and controlled; therefore the desired thickness can be obtained.

\subsubsection{Chemical synthesis of levelers}

In order to have a better leveling effect for the top surface of the deposits, we have been designing and synthesizing other levelers based on the effect of the functional groups. The overall goal of this side project is to synthesize amine and quaternary ammonium-based levelers and test its leveling effect of electrodeposition of copper for metamaterial fabrication. The synthesized levelers can be used in the electrodeposition experiments in the near future. There are two basic requirements for levelers. First, since the electrolyte used for electrodeposition is aqueous solution, levelers must have a high solubility in water. Secondly, there should be amine or quaternary ammonium functional groups in levelers.

We have successfully synthesized two candidate levelers and both of them are tertiary amine-based polyesters. The synthetic scheme of the first polyester, poly $(1,8-$ octanediol-co-dipicolinate), was shown in Scheme 2.1. Dipicolinic acid (2.06 g, 12.3 


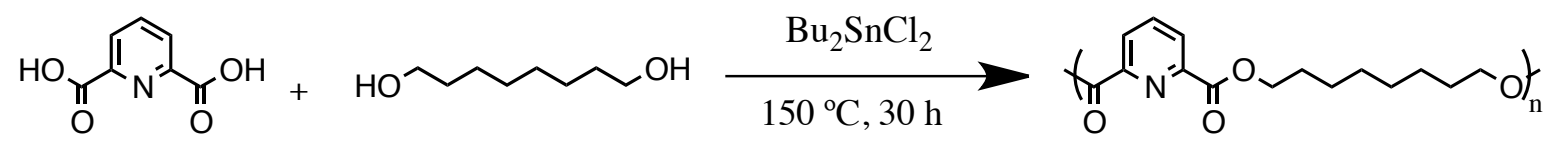

Scheme 2.1. Synthetic route for tertiary amine-based polyester, poly(1,8-octanediol-codipicolinate). Two monomers used in this polymerization reaction are dipicolinic acid and 1,8-octanediol.

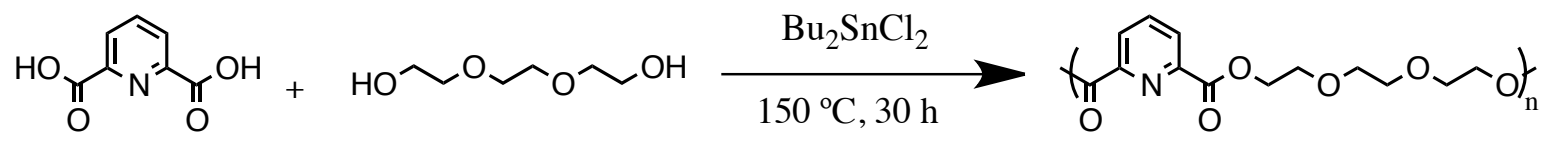

Scheme 2.2. Synthetic route for tertiary amine-based polyester, poly(triethylene glycolco-dipicolinate). Two monomers used in this polymerization reaction are dipicolinic acid and triethylene glycol.

mmol), 1,8-octanediol (2 g, $13.7 \mathrm{mmol})$ and the catalyst $\mathrm{Bu}_{2} \mathrm{SnCl}_{2}(0.04 \mathrm{~g}, 0.14 \mathrm{mmol})$ were mixed together. The mixture was stirred at $150{ }^{\circ} \mathrm{C}$ and the pressure was then decreased from atmosphere to $0.03 \mathrm{~mm} \mathrm{Hg}$. After $30 \mathrm{~h}$, a solid yellow polyester was produced. The polyester poly(1,8-octanediol-co-dipicolinate) was confirmed by proton NMR and the molecular weight is $1400 \mathrm{~g} / \mathrm{mol}$ measured with DOSY NMR. However, this polyester is very hydrophobic, so it is hard to use it as leveler in the aqueous electrolyte. In order to increase the hydrophilicity of the tertiary aminebased polyester, we replace the monomer 1,8-octanediol with triethylene glycol, which has a better solubility in water. The synthetic scheme of the second polyester was shown in Scheme 2.2. Dipicolinic acid (2.06 g, $12.3 \mathrm{mmol})$, triethylene glycol (2 g, $13.3 \mathrm{mmol})$ and $\mathrm{Bu}_{2} \mathrm{SnCl}_{2}(0.04 \mathrm{~g}, 0.14 \mathrm{mmol})$ were stirred together at $150{ }^{\circ} \mathrm{C}$ under the vacuum of $0.03 \mathrm{~mm} \mathrm{Hg}$. After $30 \mathrm{~h}$, a brownish gel was produced and confirmed as poly(triethylene glycol-co-dipicolinate) by proton NMR. The molecular weight is 1100 g/mol measured with DOSY NMR. Poly(triethylene glycol-co-dipicolinate) has a high solubility in water, so it can be used as a candidate leveler in the bottom-up electrodeposition of copper. 


\subsection{Experimental measurements}

In this research, one of the main aims was to characterize the copper deposits in order to understand the interrelationship of the electrodeposition process with the experimental parameters such as composition of electrolyte, substrate geometry, and the degradation of additives. The substrates and copper deposits were examined by advanced characterization tools: cyclic voltammetry (CV), linear sweep voltammetry (LSV), atomic force microscopy (AFM), scanning electron microscope (SEM), optical microscope, optical profilometry, four-point probe measurements, nuclear magnetic resonance spectroscopy (NMR), fourier transform infrared spectroscopy (FTIR) and terahertz time-domain spectroscopy (THz-TDS).

\subsection{1 i-E characterization}

Cyclic voltammetry (CV) and linear sweep voltammetry (LSV) are widely used to study electrochemical processes such as metal electrodeposition and corrosion and to gain insight into the phenomena occurring at the interface between the electrode and the electrolytic solution in the presence of additives. All the CV and LSV experiments were carried out in a conventional three-electrode cell using a Gamry (PCI4/300) potentiostat. The potentiostat was calibrated with a dummy cell before electrochemical experiments.

\subsubsection{Surface topography}

An optical microscope (Nicolet 0049-005) was used to characterize the topography of the samples received from the electrodeposition process in order to analyze the effects and interplay of additives. The atomic force microscopy (Agilent Technologies 5420) examination was conducted using PicoView under contact mode so as to investigate the morphology of the samples received from the electrodeposition process, and to study the mechanism of nucleation and growth of copper. The AFM tip (Nanoandmore USA, HQ:CSC17/Al BS) was $15 \mu \mathrm{m}$ in height with the backside 


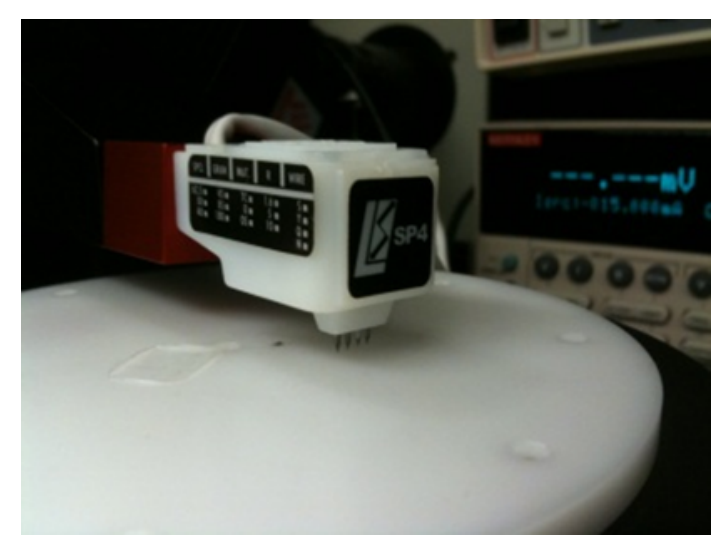

Figure 2.5. Setup of four-point probe device.

coated with $\mathrm{Al}$ (coating thickness $\approx 30 \mathrm{~nm}$ ). The force constant of AFM cantilever (10 $\mu \mathrm{m}$ wide by $450 \mu \mathrm{m}$ long by $2 \mu \mathrm{m}$ thick) is $0.18 \mathrm{~N} / \mathrm{m}$. An optical profilometer (Vecco NT 9109) was used to measure the surface topography, the RMS surface roughness and to acquire 3D images. It can provide a very fast measurement over large lateral areas.

\subsubsection{Conductivity measurements}

A four-point probe instrument (Signatone S-302-4), shown in Fig. 2.5, was used to characterize the sheet resistance of the copper film deposited under different conditions. The spacing of each probe was $1.016 \mathrm{~mm}$. Since the film thickness $(150 \mu \mathrm{m})$ was less than half the probe spacing, the resistivity can be calculated using Eqn. 2.1.

$$
\rho=4.5324 \frac{V}{i} t
$$

where 4.5324 is a constant based on sample geometry and probe spacing, $V$ is the voltage measured, $i$ is the current sourced and $t$ is the sample thickness in $\mathrm{cm}$. Four different positions were measured for each sample. For each position, the applied current was from $1 \mathrm{~A}$ to $-1 \mathrm{~A}$ with an interval of $0.2 \mathrm{~A}$. The corresponding voltages were averaged over four voltage readings. 


\subsubsection{Chemical analysis}

\subsubsection{Proton-NMR}

Proton-NMR (300 MHz and $600 \mathrm{MHz}$, Bruker) was used to investigate the chemical structures of the additives and the changes in their concentrations. The ${ }^{1} \mathrm{H}-\mathrm{NMR}$ samples were prepared via the following steps [41]: (1) $8 \mathrm{M} \mathrm{KOH}$ solution was used to neutralize the acid solution; (2) pure ethanol was added in a solution:ethanol ratio of 2:3 to precipitate the inorganic compounds; (3) the resulting solution was filtered and evaporated to remove the precipitate; (4) the residue was dissolved in deuterium oxide $\left(\mathrm{D}_{2} \mathrm{O}\right)$ as the solvent for the NMR measurement.

\subsubsection{Diffusion NMR}

Diffusion-ordered spectroscopy (DOSY) is an NMR technique used to measure the molecular weight of polymers. DOSY is based on the linear correlation between the logarithm of diffusion coefficient $(\log D)$ and the molecular weights $(\log M W)$, thus external calibration curves were created to give predictions of molecular weights of polymers. DOSY relates the chemical shifts of NMR resonances from a molecular species to the translational diffusion coefficient of that species, therefore, offer a facile method for accurate determination of molecular weights. Compared to Gel Permeation Chromatography (GPC), DOSY can provide a faster and cheaper measurement and it can measure the polymers of high molecular weight as well as small molecule $\left(\sim 10^{2} \mathrm{~g} / \mathrm{mol}\right)$

First, a synthesized polymer sample was prepared as usual for proton NMR, a proton spectrum was then obtained and the chemical shift scale was calibrated. Then the spinning was stopped, the diffusion experiment was loaded and a new spectrum with $5 \%$ gradient strength was obtained. The next new spectrum was obtained with $95 \%$ gradient strength. After data processing, the diffusion coefficient data was obtained, therefore the corresponding molecular weight can be interpolated from a calibration 
curve of diffusivity vs.molecular weight. Since we use dipicolinic acid as the backbone for the synthesized polymer, the external calibration curve is chosen from literature regarding benzene ring based polymers [42].

\subsubsection{FTIR}

An FTIR spectrometer (Nexus 670 ThermoNicolet) was also used to identify the chemical structure of the additives. The powder that was extracted from the electrolyte was mixed with $\mathrm{KBr}$, which serves as a solid solvent. The concentration of the sample in $\mathrm{KBr}$ should be in the range of $0.2 \%$ to $1 \%$ because a high concentration would cause too much noise for the peaks while a low concentration results in a weak signal, which is hard to visualize in both cases. A $1 \mathrm{mg}$ powder sample and $100 \mathrm{mg}$ $\mathrm{KBr}$ were mixed together and the mixture was very finely ground in a mortar to reduce the particle size. Otherwise, large particles scatter the IR beam and cause a sloped spectrum baseline. Then a small amount of the mixture was put into a stainless steel die set, and the top part was screwed in and fastened with a wrench. Finally the top part of the die was unscrewed and a transparent pellet was obtained. A pure $\mathrm{KBr}$ pellet was made and examined by the FTIR instrument first to get a background $\mathrm{KBr}$ spectrum, then the unknown powder/KBr pellet was examined. All the FTIR spectra were acquired in absorption mode.

\subsubsection{Terahertz time-domain spectroscopy (THz-TDS)}

As shown in Fig. 2.6 and Fig. 2.7, the THz-TDS system is equipped with an erbium-doped Raman-shifted fiber laser (IMRA Femtolite FX-100) operating at a central wavelength of $805 \mathrm{~nm}$. The laser produces $\leq 120 \mathrm{fs}$ free-space pules at a repetition rate of $80 \mathrm{MHz}$ with an average power of $100 \mathrm{~mW}$. The laser pulse travels through a beam sampler and split into two portions. The primary portion propagates though a time delay and strikes a photo-conductive antenna (PCA) that generates terahertz radiation. The terahertz radiation interacts with the metamaterial sample 


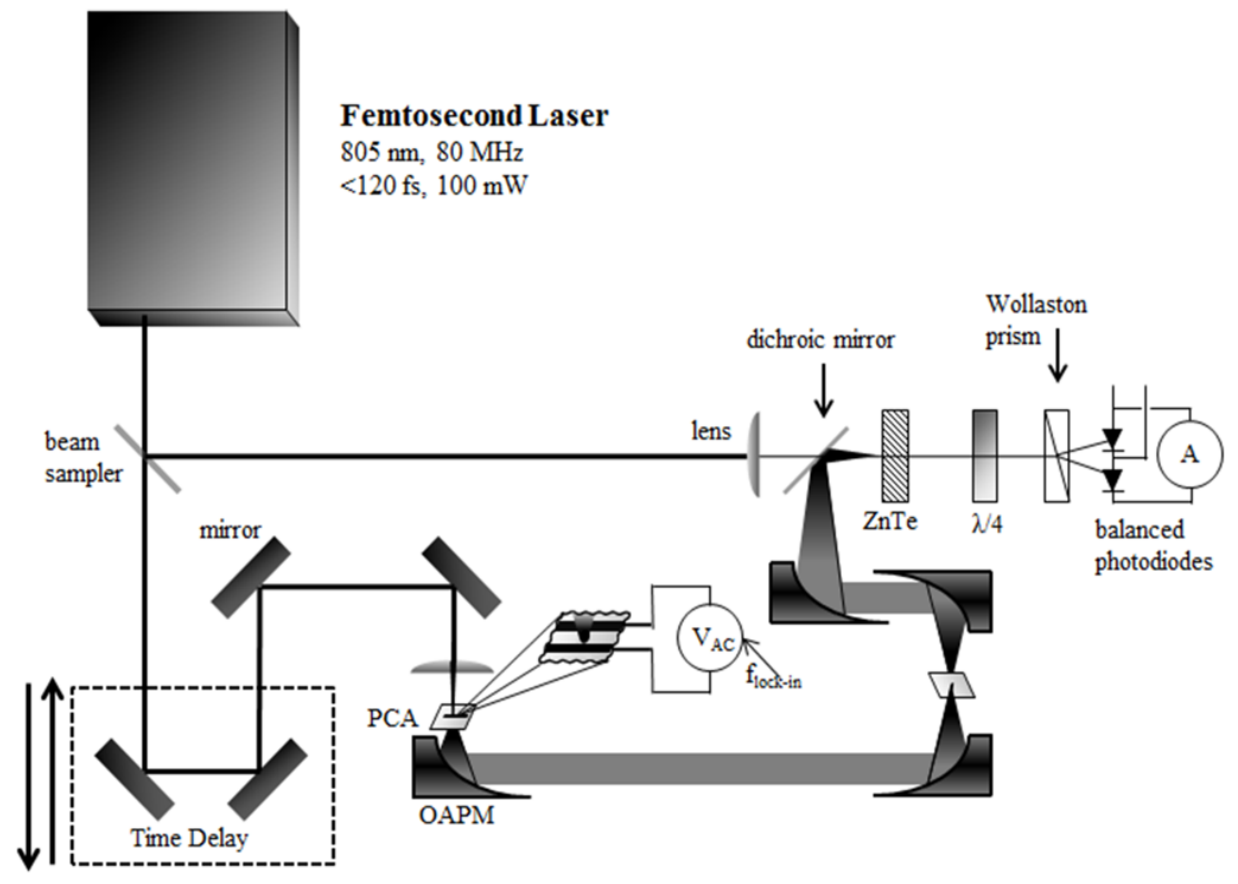

Figure 2.6. Schematic of terahertz time-domain spectroscopy.

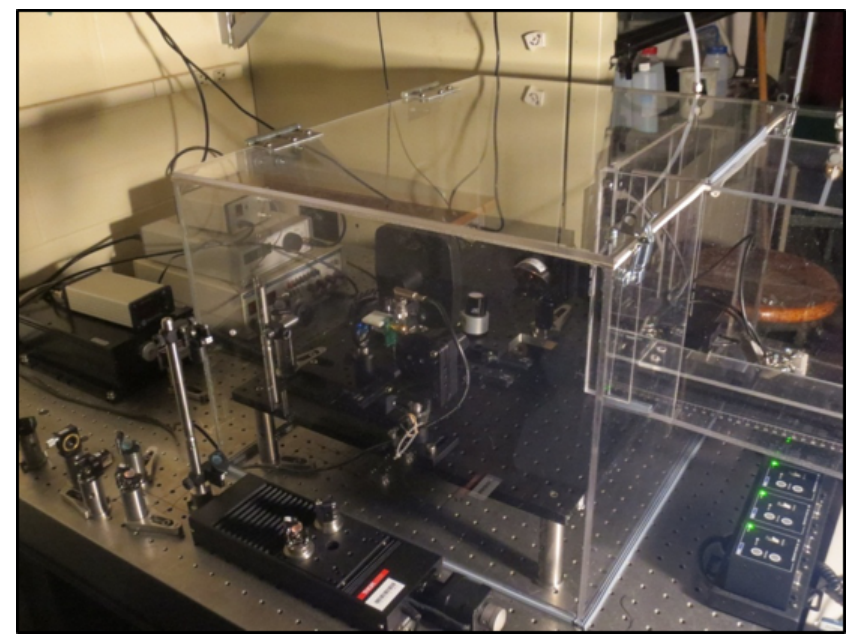

Figure 2.7. Setup of terahertz time-domain spectroscopy system. 
and the resulting beam meet with the second portion of the laser pulse. After the combined beam is focused onto a nonlinear crystal, $\langle 110\rangle \mathrm{ZnTe}$, the polarization of the probe beam is then shifted proportional to the electric field strength of the terahertz pulse. In order to measure the shift in polarization, the probe beam is split with a Wollaston prism into two orthogonally polarized portions. Each portion is absorbed by a photodiode whose voltage difference is measured with a lock-in amplifier. The lock-in amplifier measures with a time constant of $300 \mathrm{~ms}$ at a repetition rate of $5 \mathrm{kHz}$. To measure the full terahertz pulse, the time delay is moved in steps of $5 \mu \mathrm{m}$ with the electric field strength measured at each step.

\subsection{Results and Discussion}

\subsubsection{Cyclic voltammetry}

The cyclic voltammogram of the electrolyte used for electrodeposition is shown in Fig. 2.8, the predominant copper ion in this solution is $\mathrm{Cu}^{2+}$, however, a small amount of $\mathrm{Cu}^{+}$may be present in the solution due to the multistep charge reaction: $\mathrm{Cu}^{2+}+\mathrm{e}^{-} \longrightarrow \mathrm{Cu}^{+}$.

Table 2.1. Standard reduction potentials of copper ions in aqueous solution at $25{ }^{\circ} \mathrm{C}$.

\begin{tabular}{ccc}
\hline Half reaction & $E^{\circ}(\mathrm{V}$ vs. SHE $)$ & $E^{\circ}(\mathrm{V}$ vs. $\mathrm{Ag} / \mathrm{AgCl})$ \\
\hline $\mathrm{Cu}^{+}+\mathrm{e}^{-} \longrightarrow \mathrm{Cu}(\mathrm{s})$ & 0.52 & 0.3 \\
$\mathrm{Cu}^{2+}+2 \mathrm{e}^{-} \longrightarrow \mathrm{Cu}(\mathrm{s})$ & 0.34 & 0.12 \\
$\mathrm{Cu}^{2+}+\mathrm{e}^{-} \longrightarrow \mathrm{Cu}^{+}$ & 0.15 & -0.07 \\
\hline
\end{tabular}

Table 2.1 shows the standard reduction potentials of copper ions in aqueous solution at $25{ }^{\circ} \mathrm{C}$. As is known, the more positive the reduction potential, the greater the tendency for the species to be reduced. As the scanning of CV goes from positive to negative, the sequence of the reduction reaction is $\mathrm{Cu}^{+}+\mathrm{e}^{-} \longrightarrow \mathrm{Cu}(\mathrm{s})$ (first), $\mathrm{Cu}^{2+}+2 \mathrm{e}^{-} \longrightarrow \mathrm{Cu}(\mathrm{s})$ (second), $\mathrm{Cu}^{2+}+\mathrm{e}^{-} \longrightarrow \mathrm{Cu}^{+}$(third). Since the predominant valence state of copper in the electrolyte is $\mathrm{Cu}^{2+}$, the major reduction 


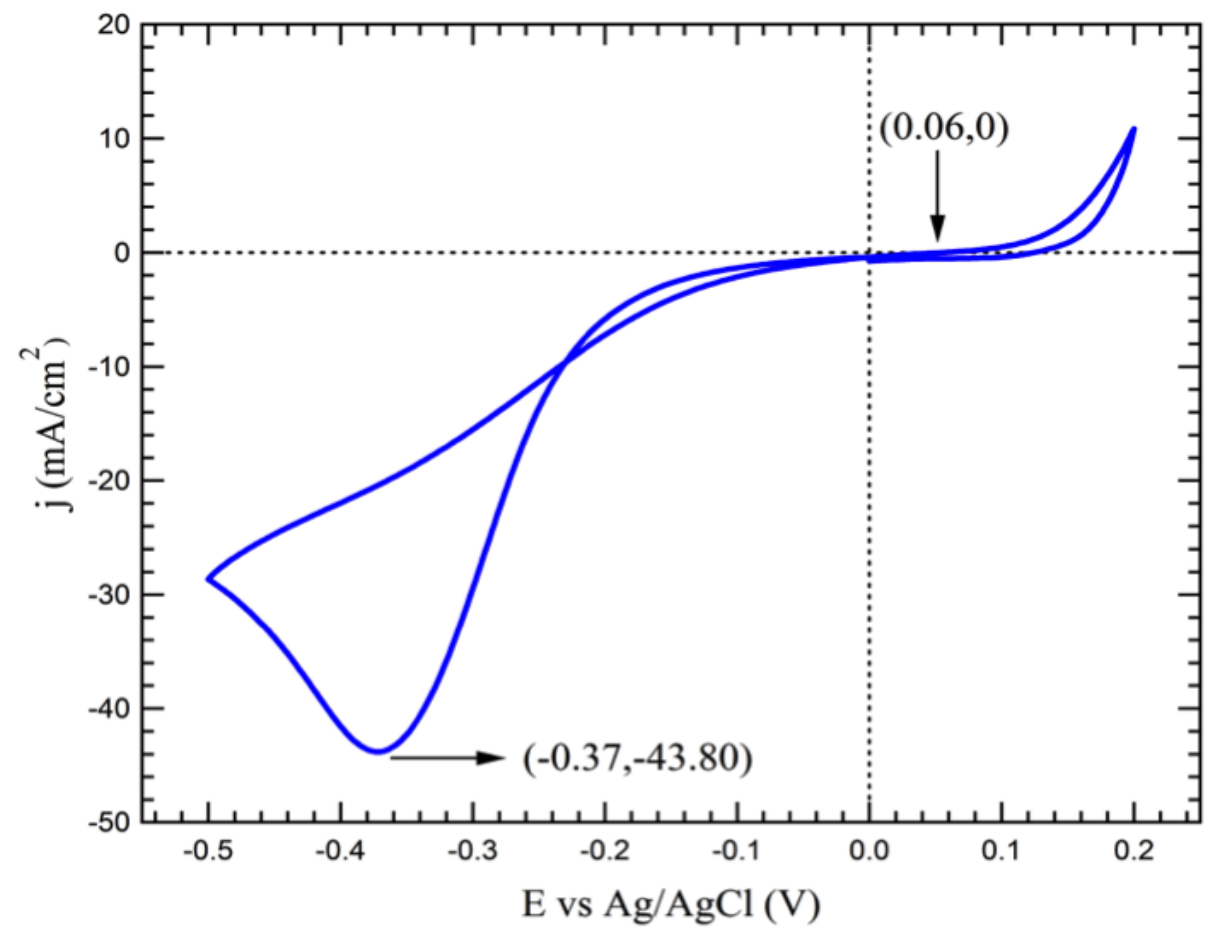

Figure 2.8. Cyclic voltammogram of the electrolyte consisting of $0.25 \mathrm{M} \mathrm{CuSO}_{4}, 1.8 \mathrm{M}$ $\mathrm{H}_{2} \mathrm{SO}_{4}, 0.3 \mathrm{~g} / \mathrm{L}$ PEG, $10^{-5} \mathrm{M}$ MPSA, $10^{-3} \mathrm{M} \mathrm{Cl}^{-}$and $20 \mathrm{mg} / \mathrm{L}$ PVP. Scan rate was 30 $\mathrm{mV} / \mathrm{s}$.

reaction is $\mathrm{Cu}^{2+}+2 \mathrm{e}^{-} \longrightarrow \mathrm{Cu}(\mathrm{s})$, whose reduction peak is at $-0.37 \mathrm{~V}$, as shown in Fig. 2.8. The standard reduction potential for $\mathrm{Cu}(\mathrm{II}) / \mathrm{Cu}(0)$ couple, $E^{\circ}$, is $0.12 \mathrm{~V}$ vs. $\mathrm{Ag} / \mathrm{AgCl}$, as shown in Table 2.1. The onset of $\mathrm{Cu}$ reduction on the working electrode begins at $0.06 \mathrm{~V}$, approximately $0.06 \mathrm{~V}$ negative of $E^{\circ}$ (Fig. 2.8).

\subsubsection{Linear sweep voltammetry}

A series of linear sweep voltammetry experiments were carried out to investigate the electrochemical behavior of additives. The electrolyte was acid copper bath with different combination of additives. The scan range was from 0 to $-0.25 \mathrm{~V}$. The scan rate was $30 \mathrm{mV} / \mathrm{s}$.

In Fig. 2.9, the red curve represents the LSV curve of acid copper solution and the blue curve is the LSV curve of acid copper solution with Cl-PEG. Fig. 2.9 shows that 


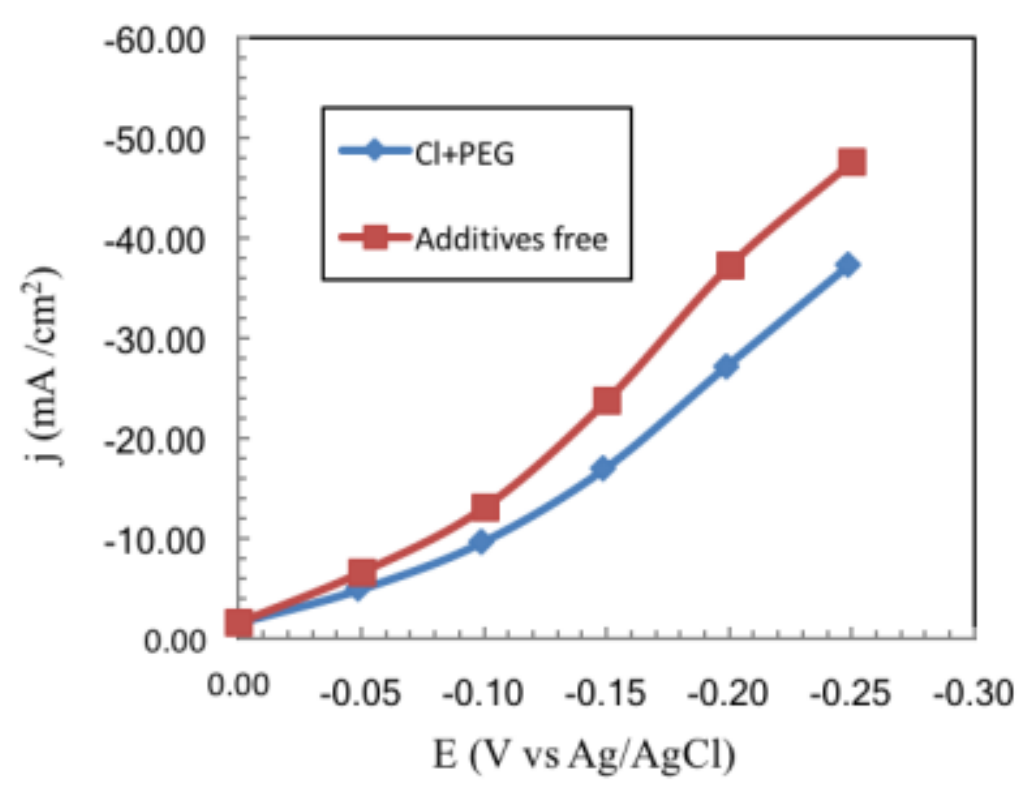

Figure 2.9. LSV curves obtained in additive-free electrolyte and electrolyte with $\mathrm{Cl}^{-}$and PEG, respectively.

the current density is decreasing with the addition of chloride ion and PEG, indicating that the combination of Cl-PEG suppresses that process of electrodeposition.

A series of LSV experiments for PEG-MPSA-Cl electrolyte with different concentrations of $\mathrm{Cl}^{-}$ranging from $20 \mathrm{ppm}$ to $200 \mathrm{ppm}$ are shown in Fig. 2.10, and it shows that the higher the concentration of chloride ion, the higher the current density, meaning that the presence of $\mathrm{Cl}^{-}$promotes the process of electrodeposition.

Comparing the LSV results got from Fig. 2.9 and Fig. 2.10, it can be concluded that PEG suppresses the electrodeposition process, which results from the mechanism that PEG forms a monolayer on the cathode to inhibit the fast and disordered electrodeposition. In Fig. 2.11, the red curve is the LSV curve of acid copper solution with chloride ion, PEG and MPSA, and the blue curve is the LSV curve of acid copper solution with chloride ion and MPSA. Fig. 2.11 shows that, compared to the Cl-MPSA electrolyte, the current density drops upon the addition of PEG, which reveals again that PEG acts as an inhibitor during the process of electrodeposition. 


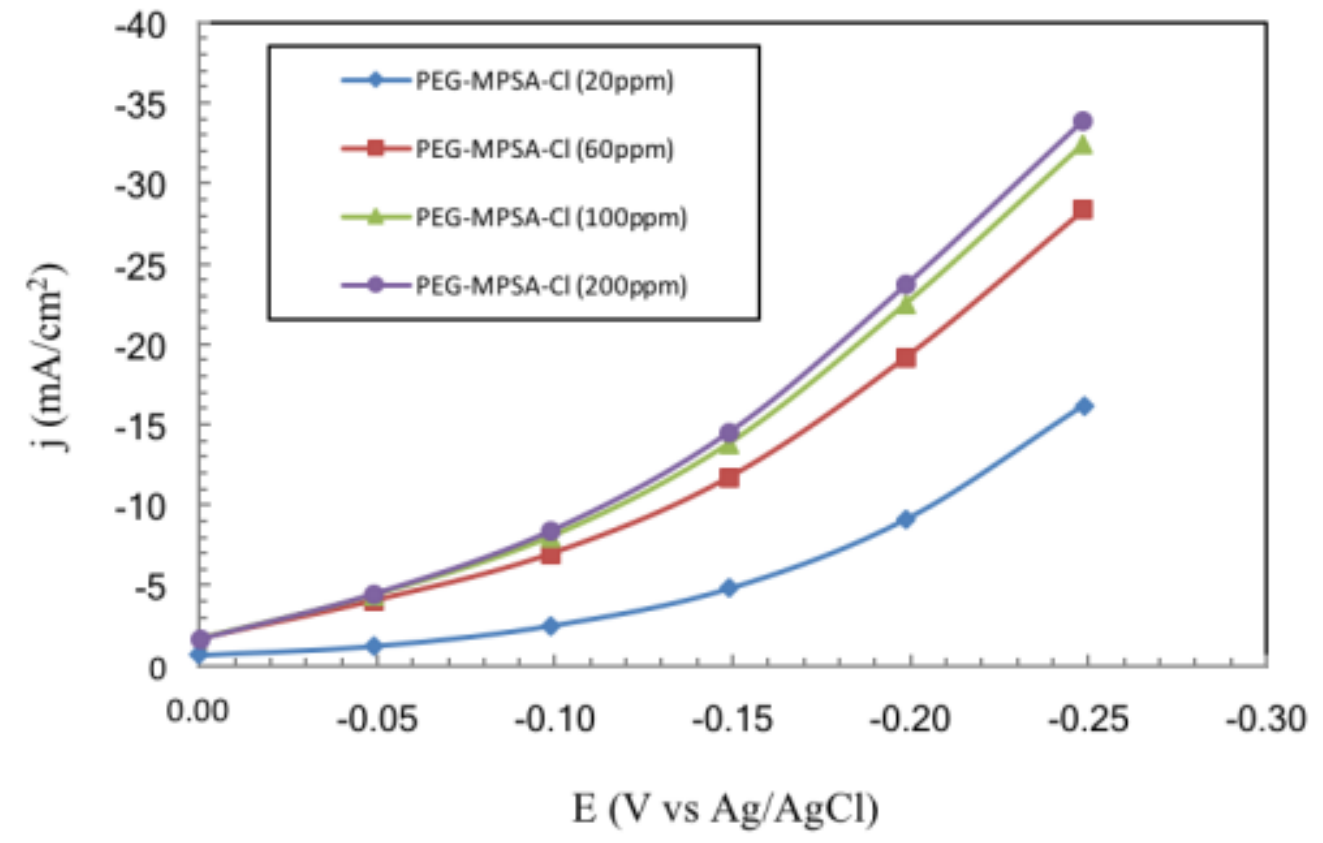

Figure 2.10. LSV curves obtained in acid copper electrolytes with PEG, MPSA and a range of $\mathrm{Cl}^{-}$.

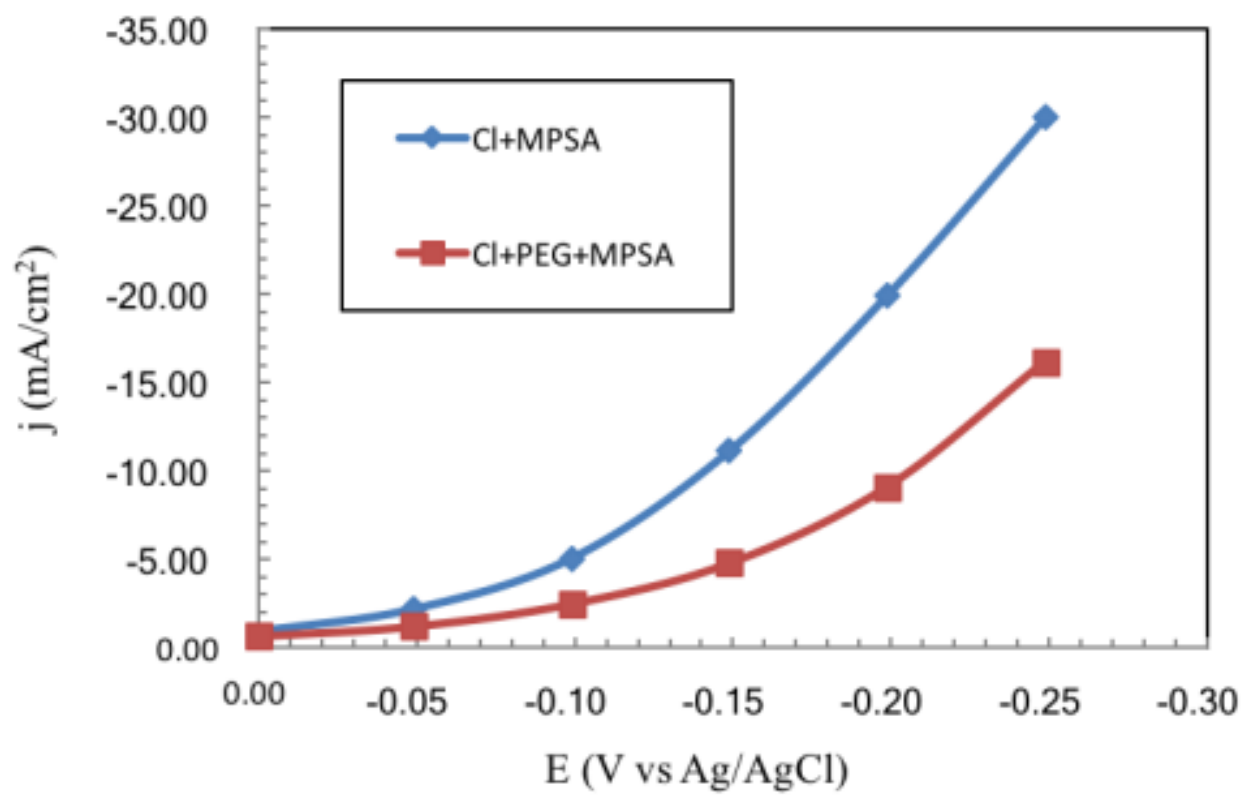

Figure 2.11. LSV curves obtained from acid copper electrolytes with and without PEG, respectively. 


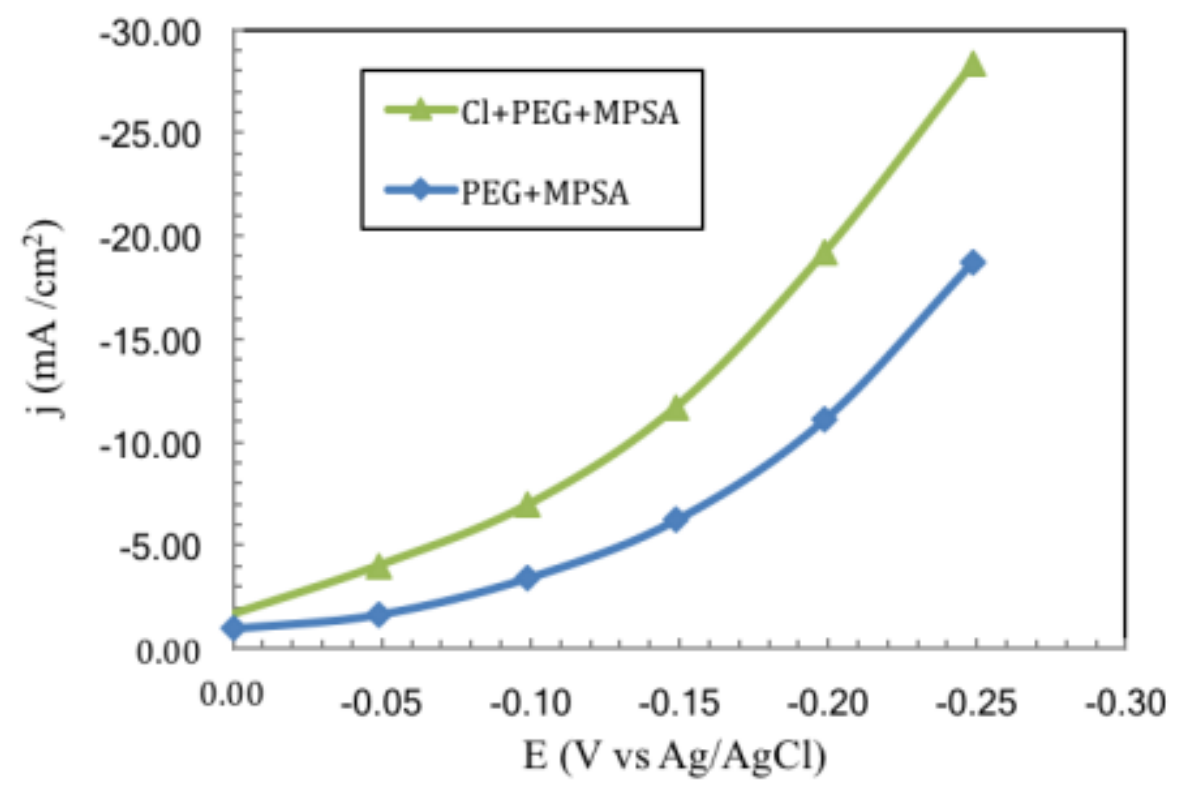

Figure 2.12. LSV curves obtained in acid copper electrolytes with and without $\mathrm{Cl}^{-}$, respectively.

Besides, the overall current density of Cl-PEG-MPSA electrolyte is higher than that of PEG-MPSA electrolyte as shown in Fig. 2.12, which illustrates that in the presence of $\mathrm{Cl}^{-}$, the current density increases and even a small amount of $\mathrm{Cl}^{-}$leads to rapid transition of PEG-MPSA electrolyte from suppression to acceleration of electrodeposition. The functions of chloride ion and PEG are consistent with previous research $[21,22,31,35,36,43-47]$.

Fig. 2.13 shows a series of LSV tests of Cl-PEG with and without MPSA. The general current density of Cl-PEG decreases with the increase of MPSA and the electrodeposition in acidic copper electrolytes was inhibited in the presence of MPSA and the suppression effect is strengthened with the increase of $\frac{[\mathrm{MPSA}]}{\left[\mathrm{Cl}^{-}\right]}$, as shown in Fig. 2.13. 


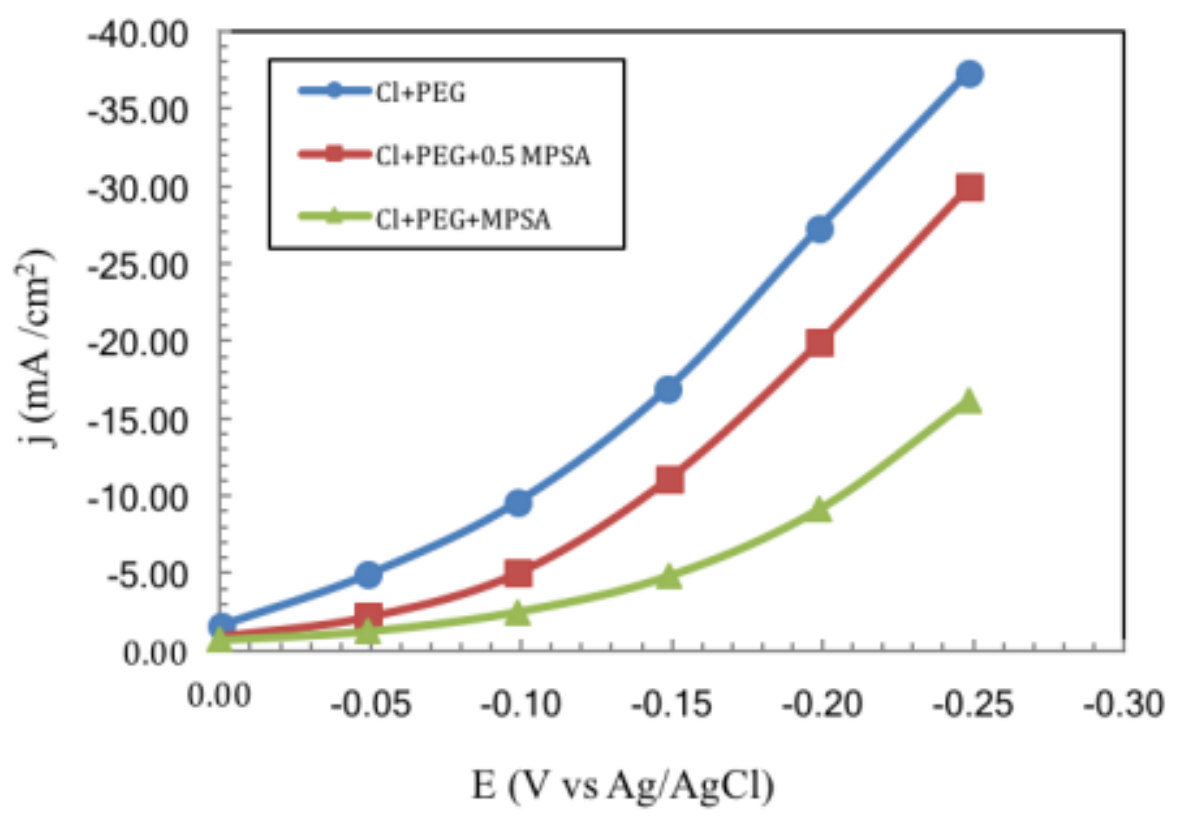

Figure 2.13. LSV curves obtained in acid copper electrolytes with Cl-PEG and a range of MPSA.

\subsubsection{Electrodeposition of copper}

Fig. 2.14 shows the electrodeposition profiles under different controlled potentials are similar, with each having a gradual increase of current density for the first $0.5 \mathrm{ks}$, then reaching a steady state current density. According to some previous studies, as the thickness of copper increases from a few nanometers to $500 \mathrm{~nm}$, the resistivity of copper film decreases from $20 \mu \Omega \mathrm{cm}$ to the resistivity of bulk copper, $1.7 \mu \Omega \mathrm{cm}$, and then remains unchanged $[48,49]$. In our study, the increase of the current density is because the resistivity of thin copper film decreases as the thickness of the copper film increases during the electrodeposition process, until the resistivity of deposited copper is close to that of bulk copper. Fig. 2.15c shows a fine, smooth, and bright mirror-like copper surface when the applied potential was $-0.08 \mathrm{~V}$ with a $14 \mathrm{~mA} / \mathrm{cm}^{2}$ steady state current density (Fig. 2.14). However, lower or higher voltages give rise to rough and dull copper films. As we know, the cyclic voltammogram (Fig. 2.8) can provide a general potential and current density range for the electrodeposition of 


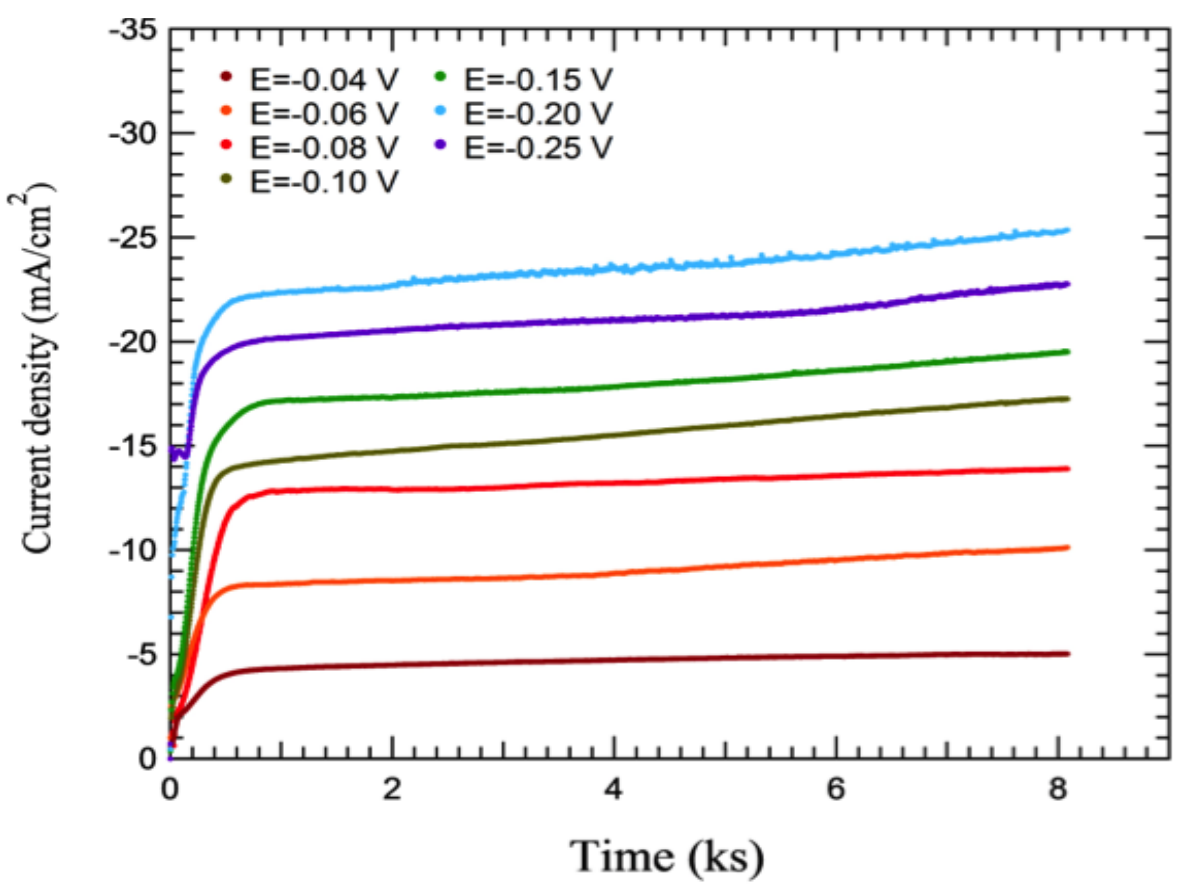

Figure 2.14. Comparison of controlled potential electrodepositions in Cl-PEG-PVPMPSA electrolyte under different potentials ranging from -0.04 to $-0.25 \mathrm{~V}$.

desired species in the electrolyte. The current density during electrodeposition has a large effect on the surface roughness and grain structure of the film. In general, the larger the current density the finer the grain refinement [50]. However, an increase in the current density results in a higher overpotential that increases the nucleation rate, thus resulting in a higher degree of roughness. Therefore, there is an optimal current density for a typical electrodeposition of metal. In Fig. 2.15 a-c , a more negative potential, and therefore higher current density, give rise to a finer surface. As the potential and current density is increased further clusters begin to form due to extreme nucleation rates as seen in Fig. 2.16. The corresponding outline images were generated by Image $\mathrm{J}$ and the analysis summary is shown in Table 2.2. As we increase the voltage, the total number of clusters increases, while the size of the clusters decreases. However, panel (f) has the highest deposition total area of the 


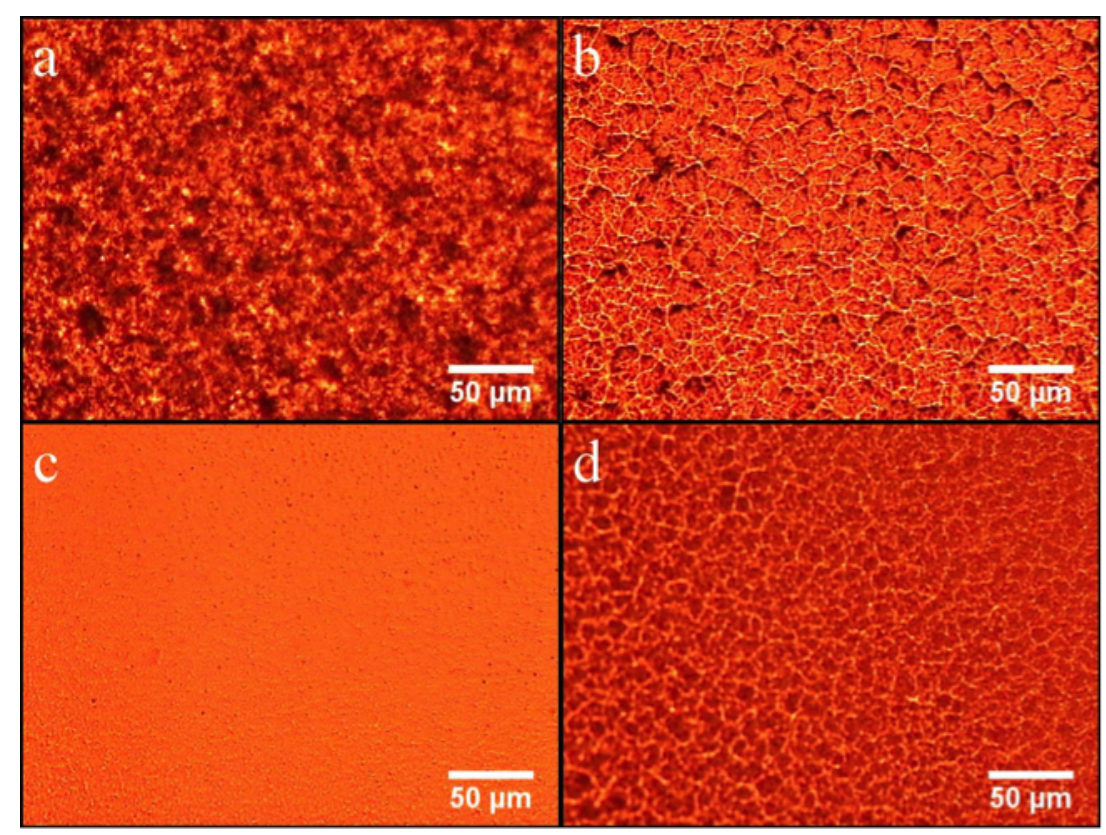

Figure 2.15. Optical microscope images of copper film electrodeposited under different potentials in Cl-PEG-PVP-MPSA electrolyte. From a to d, the potentials are $-0.04,-0.06$, -0.08 and $-0.10 \mathrm{~V}$, respectively.

clusters indicating that $-0.2 \mathrm{~V}$ gives rise to a highest deposition rate and current density, which agrees with the current profile in Fig. 2.14.

Table 2.2. Analysis summary of the surface topography of copper film electrodeposited under different potentials in Cl-PEG-PVP-MPSA electrolyte. From e to g, the potentials are $-0.15,-0.20$, and $-0.25 \mathrm{~V}$, respectively. Images correspond to Fig. 2.16.

\begin{tabular}{ccccc}
\hline Image No. & Count & Total Area $\left(\mu^{2}\right)$ & Average Size $\left(\mu^{2}\right)$ & Area Fraction $(\%)$ \\
\hline e & 42 & 12316.345 & 293.246 & 16.4 \\
$\mathrm{f}$ & 106 & 27131.074 & 255.95 & 36.2 \\
$\mathrm{~g}$ & 232 & 19216.145 & 82.828 & 25.6 \\
\hline
\end{tabular}

The leveling effect in electrodeposition mainly results from the selective adsorption of levelers on the cathode, which is dependent on the functional groups, the molecular weight, the surface area of levelers, and even the location of the functional groups. On one hand, since a copper atom has two free electrons at the outermost shell, they can move freely along the lattices when an electric field is applied. The higher 


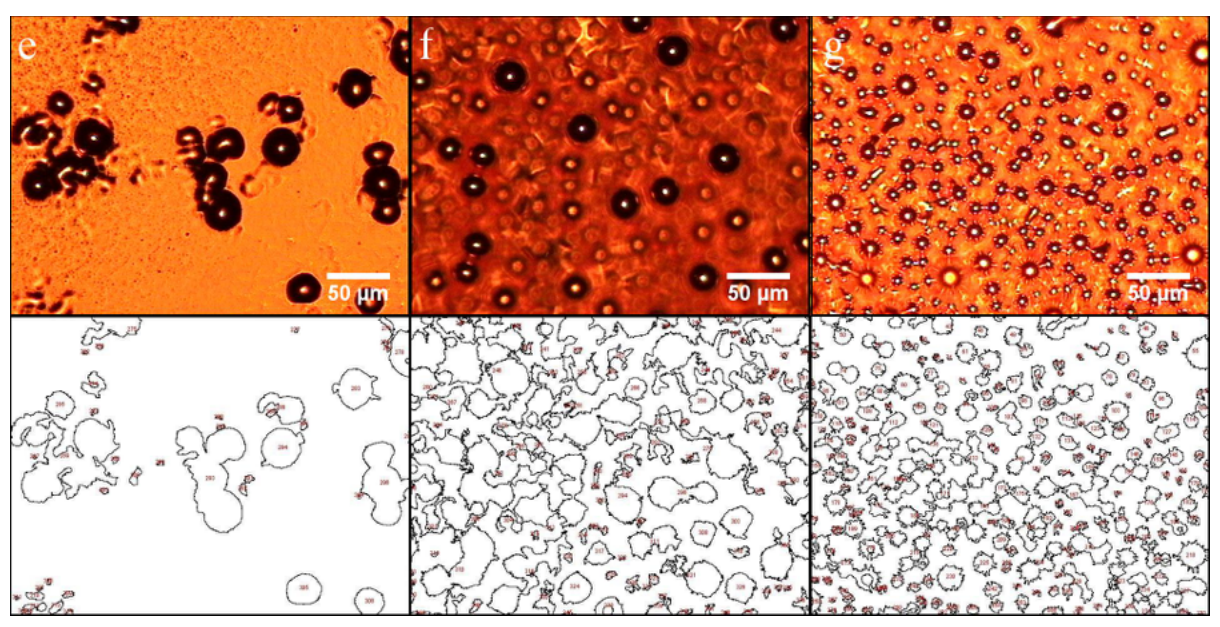

Figure 2.16. Optical microscope images of copper film electrodeposited under different potentials in Cl-PEG-PVP-MPSA electrolyte. From e to g, the potentials are $-0.15,-0.20$, and $-0.25 \mathrm{~V}$, respectively. The images in the second row are the corresponding outline drawings from the Image $\mathrm{J}$ software package.

the electric field is, the more empty $s$ bands can be formed. Therefore, amines can adsorb onto the substrate, especially on the high-current-density areas, by donating their lone pair electrons to the unoccupied $s$ bands. On the other hand, as the only non-metallic cation with a permanent positive charge, quaternary ammonium can preferentially adsorb onto the high current density area, the convex area, and block electrodeposition, resulting in a leveling effect on the cathode. Since amine and quaternary ammonium groups are the most common functional groups used in levelers, PVP and JGB were compared on the basis of their leveling capabilities. Substrates with $150 \mu \mathrm{m}$-thick 3D pillars (Fig. 2.1) were used and the total thickness of the deposited copper is $200 \mu \mathrm{m}$. As shown in Fig. 2.17, even with levelers the outer surface of the deposits was still wavy. The final height difference leveled by PVP is $17.17 \mu \mathrm{m}(8.59 \%$ of the total thickness) while that of JGB is $6.52 \mu \mathrm{m}(3.26 \%$ of the total thickness). It is concluded that JGB is a better leveling than PVP. 


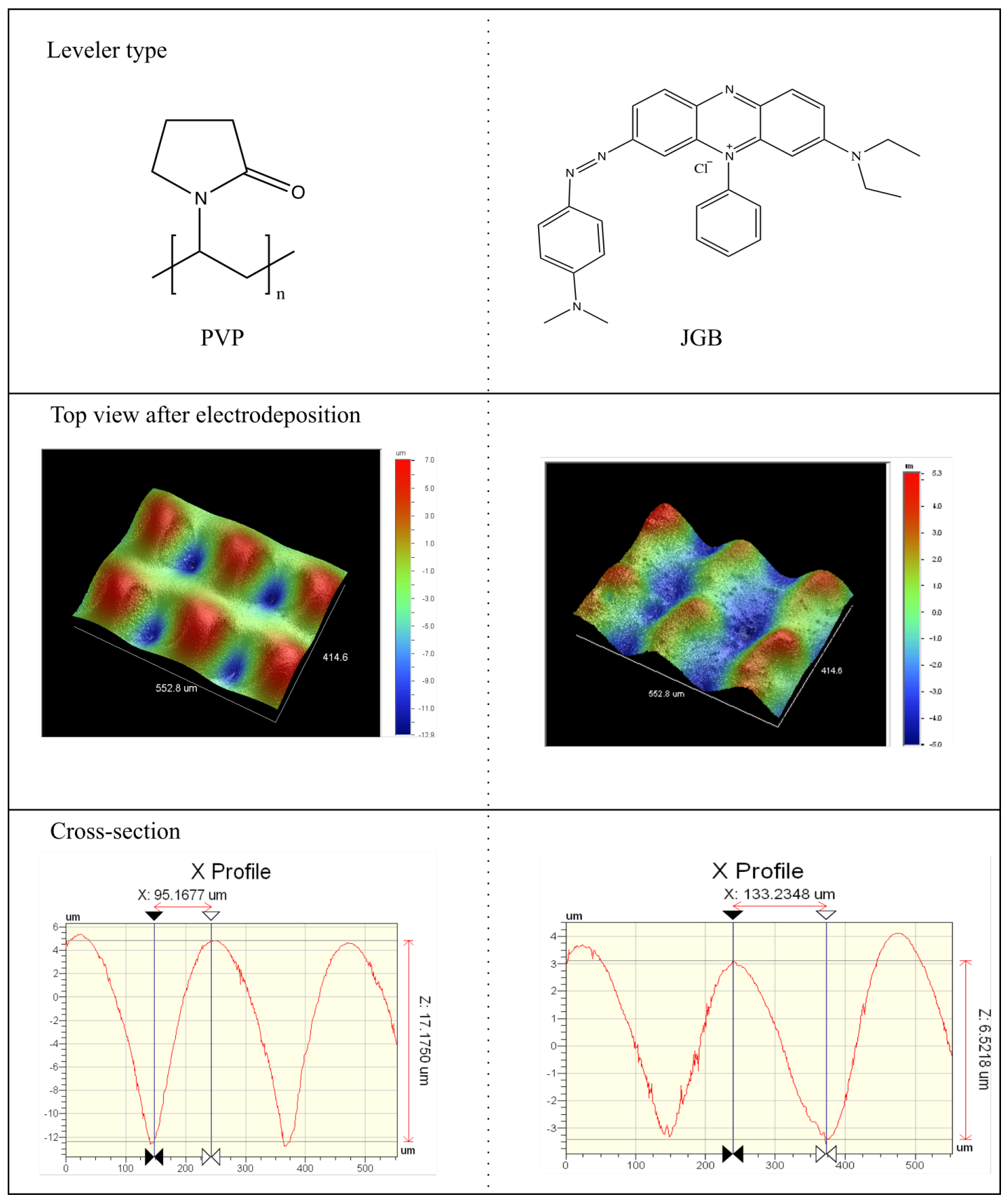

Figure 2.17. Comparison of the chemical structure and the leveling effect of PVP and JGB. 
(a)

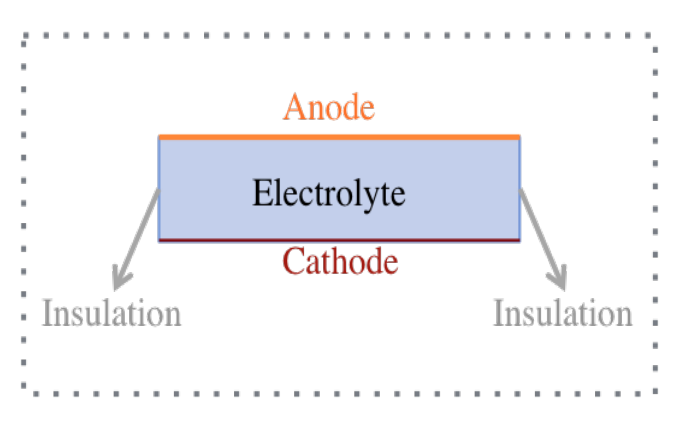

(c)

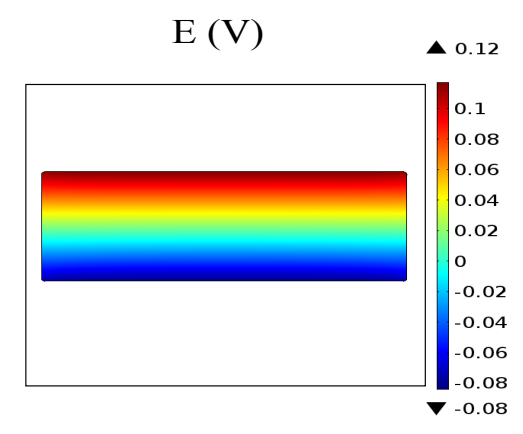

(b) The profile of deposited copper

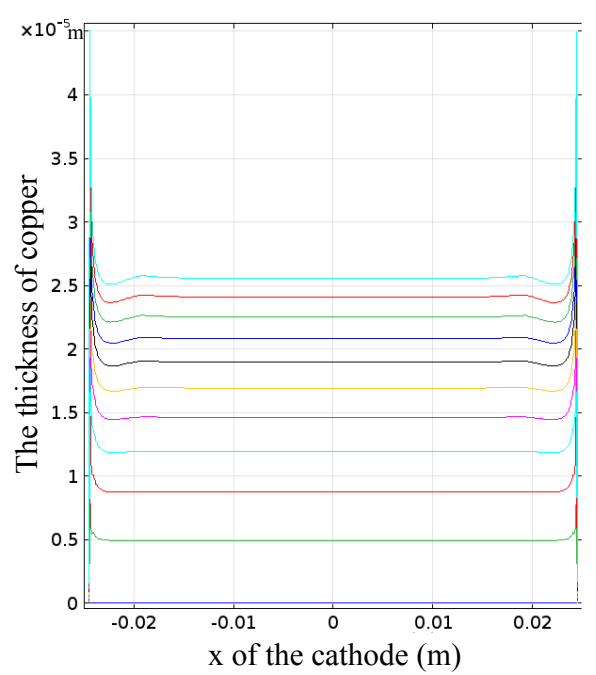

(d)

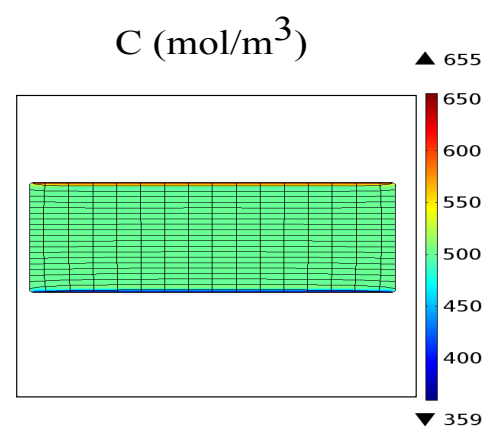

Figure 2.18. The initial COMSOL simulation of electrodeposition of copper. (a) Geometry (b) Deposition profiles ranging from $500 \mathrm{~s}$ to $5000 \mathrm{~s}$. (c) Electrolyte potential after $1 \mathrm{~min}$ electrodeposition. (d) Concentration of $\mathrm{Cu}^{2+}$ ions after 1 min electrodeposition.

\subsubsection{Finite element modeling}

Two-dimensional FEM of differing electrodeposition conditions, including variance of aspect ratio of the features, the size of the electrodes, deposition time, and the distance between the cathode and anode, have been investigated. The basic settings can be seen in the COMSOL documentation $[51,52]$.

As shown in Fig. 2.18 (a), the rectangular cell used for electrodeposition has four boundaries: the surfaces of the cathode and the anode, and two artificial edge boundaries. The length of both the cathode and anode are $5 \mathrm{~cm}$ and the distance 
between those two is $2 \mathrm{~cm}$. The acid copper electrolyte is inside the rectangle; no additives are added. Fig. 2.18 (b) is the profile of the deposited copper during the electrodeposition process. The lines represent the electrodeposition from $500 \mathrm{~s}$ to $5000 \mathrm{~s}$ with an interval of $500 \mathrm{~s}$, and the deposited film is flat across the wafer except at the edges. The electrolyte potential of the electrodeposition is uniform across the horizontal direction as shown in Fig. 2.18 (c). Besides, seen in Fig. 2.18 (d) the concentration of the copper ions remains the same as the initial concentration of the bulk electrolyte except that the concentration on the anode is higher than the bulk concentration. This is because the consumable copper anode would lose electrons and generate copper ions, while on the cathode the concentration is lower than the bulk due to the reduction of the copper ions to accomplish electrodeposition. The overall concentration on the cathode is uniform except at the edges, which is the reason for the non-uniform electrodeposition profile on the edges.

In electrodeposition experiments that use consumable anodes, the area of the anode should be two or three times as big as that of the cathode so as to provide a sufficient source of copper ions. Besides, it is better that the cathode and anode are close to each other to avoid a large potential drop. In order to make the simulation as similar as the real experiment, the length of the cathode was changed to 1 $\mathrm{cm}$, one-third that of the anode, and the distance between the two electrodes was changed to $1 \mathrm{~cm}$, as shown in Fig. 2.19. The profile of three hours' electrodeposition of copper does not show a flat surface, and some sharp peaks appear close to the boundaries (Fig. 2.19 b), but due to the presence of additives, in real electrodeposition the copper film is even across the wafer, as shown in Fig. 2.22. The comparison between the simulation and the experimental results prove again the importance of the additives. The electrolyte potential of electrodeposition of $10 \mathrm{~min}$ and $3 \mathrm{~h}$ are shown in Fig. 2.19 and it is clear that after three hours electrodeposition the potential between the cathode and anode is smaller than that of 10 minutes electrodeposition, 

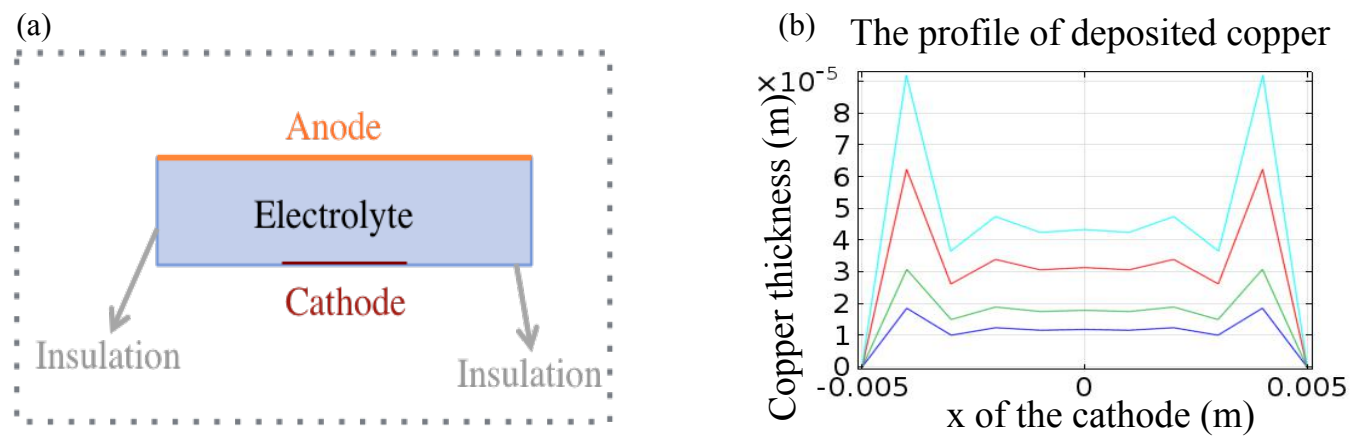

(c)

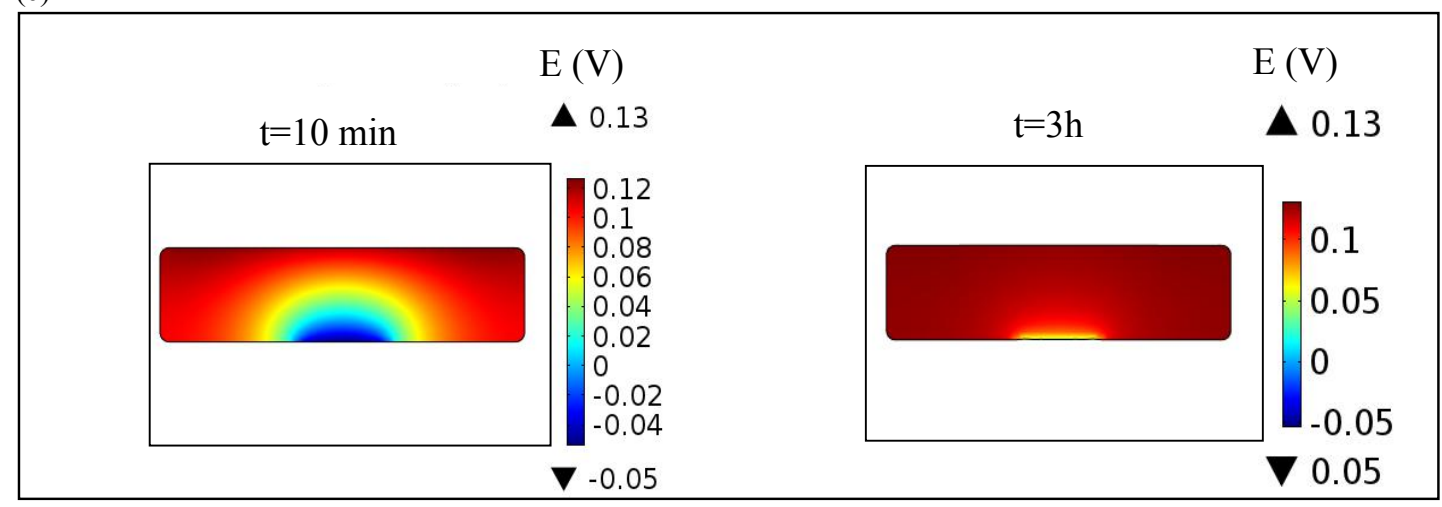

(d)

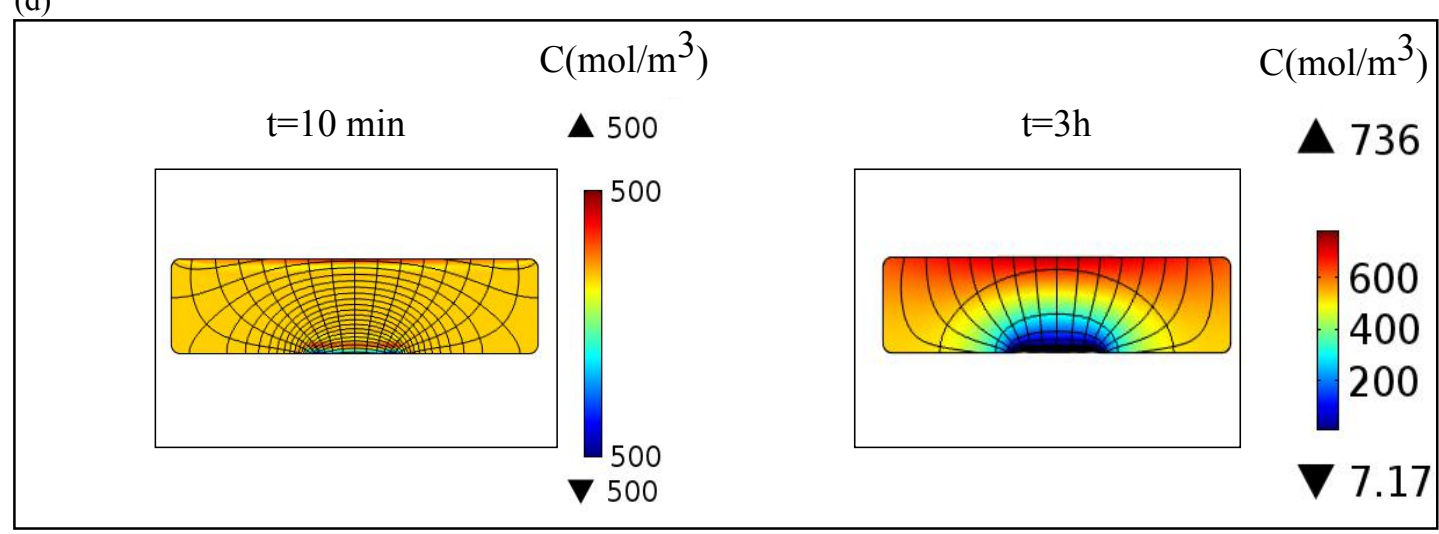

Figure 2.19. Final COMSOL simulation of electrodeposition of copper. (a) Geometry (b) Deposition profiles ranging from $10 \mathrm{~min}$ to $3 \mathrm{~h}$. (c) Comparison of electrolyte potential of electrodeposition of copper for $10 \mathrm{~min}$ and $3 \mathrm{~h}$. (d) Concentration of $\mathrm{Cu}^{2+}$ ion of electrodeposition of copper for $10 \mathrm{~min}$ and $3 \mathrm{~h}$. 


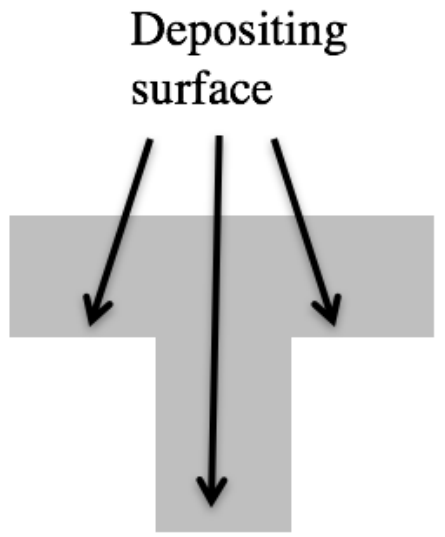

Figure 2.20. Geometry of the trench for the COMSOL simulation of superfilling.

which means the overpotential increases with the increase of the electrodeposition time. In addition, the concentration of copper ions in the electrolyte is the same as the original solution while after three hours electrodeposition, the concentration gradient appears and the concentration is highest on the anode while lowest on the cathode, as shown in Fig. 2.19.

$$
\begin{gathered}
i_{l o c}=-(0.4726 \theta+0.0374) \exp \left(-\frac{(0.5+0.25 \theta) F \eta}{R T}\right) \\
v_{n}=-\frac{i_{l o c}}{2 \mathcal{F}} \frac{M}{\rho}
\end{gathered}
$$

Since additives are used in our electrodeposition experiment and exhibit a significant improvement of the deposited film and the superfilling of features, the COMSOL simulation of the electrodeposition with the presence of one of the most important additives, accelerators, has also been investigated. The selective adsorption of the functionality of the accelerator on the metal surface is the key mechanism of the superfilling of features, so the simulation considers the adsorption of the accelerator, but no mass transfer or kinetics are involved. The geometry of the trench is shown in 
Table 2.3. Parameters used for the COMSOL simulation of superfilling.

\begin{tabular}{llll}
\hline Name & Expression & Value & Description \\
\hline$\theta_{\text {initial }}$ & 0.4 & 0.4 & Initial surface coverage \\
$i_{0}$ & $100 \mathrm{~A} / \mathrm{m}^{2}$ & $100 \mathrm{~A} / \mathrm{m}^{2}$ & Exchange current density \\
$\eta$ & $-0.152 \mathrm{~V}$ & $-0.152 \mathrm{~V}$ & Over potential \\
$\gamma$ & $1 \times 10^{-5} \mathrm{~mol} / \mathrm{m}^{2}$ & $1 \times 10^{-5} \mathrm{~mol} / \mathrm{m}^{2}$ & Density of surface sities \\
$\mathrm{cs}_{\text {initial }}$ & $\gamma \times \theta_{\text {initial }}$ & $4 \times 10^{-6} \mathrm{~mol} / \mathrm{m}^{2}$ & Initial surface concentration \\
$\rho$ & $9000 \mathrm{~kg} / \mathrm{m}^{3}$ & $9000 \mathrm{~kg} / \mathrm{m}^{3}$ & Density of depositing species \\
$M$ & $65 \mathrm{~g} / \mathrm{mol}$ & $0.065 \mathrm{~kg} / \mathrm{mol}$ & Molar mass of depositing species \\
$T$ & $298 \mathrm{~K}$ & $298 \mathrm{~K}$ & Temperature \\
$N_{e}$ & 2 & 2 & Number of electrons in reactions \\
\hline
\end{tabular}

Fig. 2.20. The simulation is based on Eqn. 2.2 and 2.3 where $\theta$ is the surface coverage of accelerators, $\mathcal{F}$ is the Faraday constant, $\eta$ is the overpotential, $R$ is the universal gas constant, and $T$ is the absolute temperature, $v_{n}$ is the normal mesh velocity of the depositing electrode, $M$ is the molar mass of depositing species, and $\rho$ is the density of depositing species. As shown in Eqn. 2.2, an initial surface coverage of accelerator was set first; the local current density can then be calculated. The normal mesh velocities can be obtained using Faraday's law as shown in Eqn. 2.3. Different normal mesh velocity on different spots of the electrode gives rise to deformation and curvature of the deposits. A deformed geometry interface is used to model the mesh movement. The model was solved using a time-dependent study with automatic remeshing. All the initial parameters are set in Table 2.3.

As shown in Fig. 2.21, copper was electrodeposited on the trenches with aspect ratios of $0.5,1$, and 2 . The images on the bottom show the corresponding profiles of electrodeposition after $30 \mathrm{~min}$, and the color shows the surface concentration of accelerator. No voids are seen in the trenches indicating the success of superfilling, however, overplating appears above the superfilled area and bumps were formed, which is a common phenomenon for electrodeposition with the addition of accelera- 


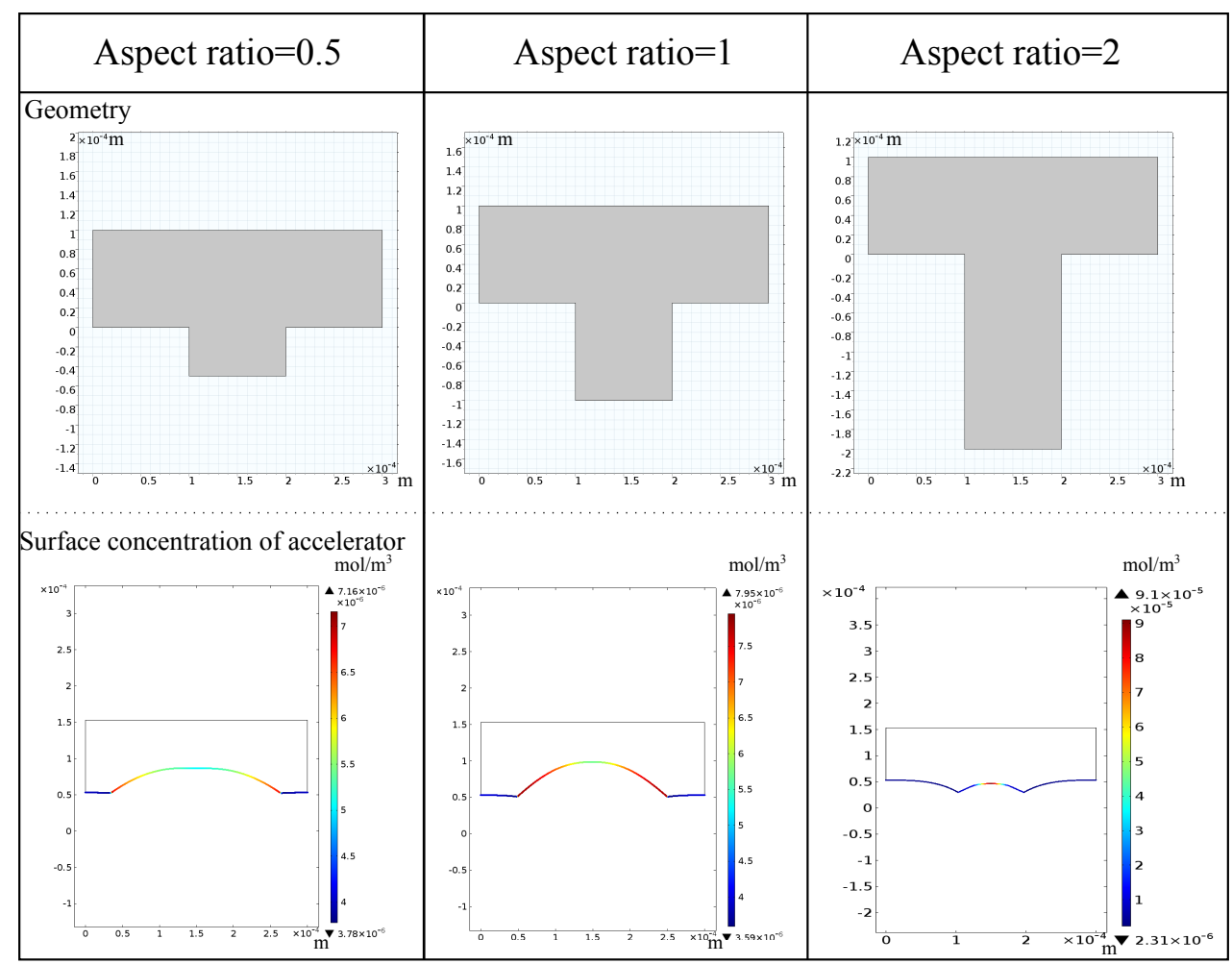

Figure 2.21. The cross-section view of the trenches with different aspect ratios (top) and the corresponding profile and surface concentration of accelerators after one hour of electrodeposition (bottom).

tors. The lateral diameter of the bumps decreases as the aspect ratio increases and the concentration of accelerators on the overplated area is higher than that of the flat area, which is caused by the accumulation of accelerators in the trenches during the superfilling process. In addition, from the highest to lowest aspect ratio, the highest concentration of accelerator represented by red in Fig. 2.21 moves from the top to the shoulder of the bumps, which means after overplating takes place, the highest accelerating changes from the vertical direction to the lateral direction.

\subsubsection{Degradation of additives}

In order to investigate the impact of electrolyte aging on the surface topography of copper films, a series of electrodepositions of copper films was carried out using 

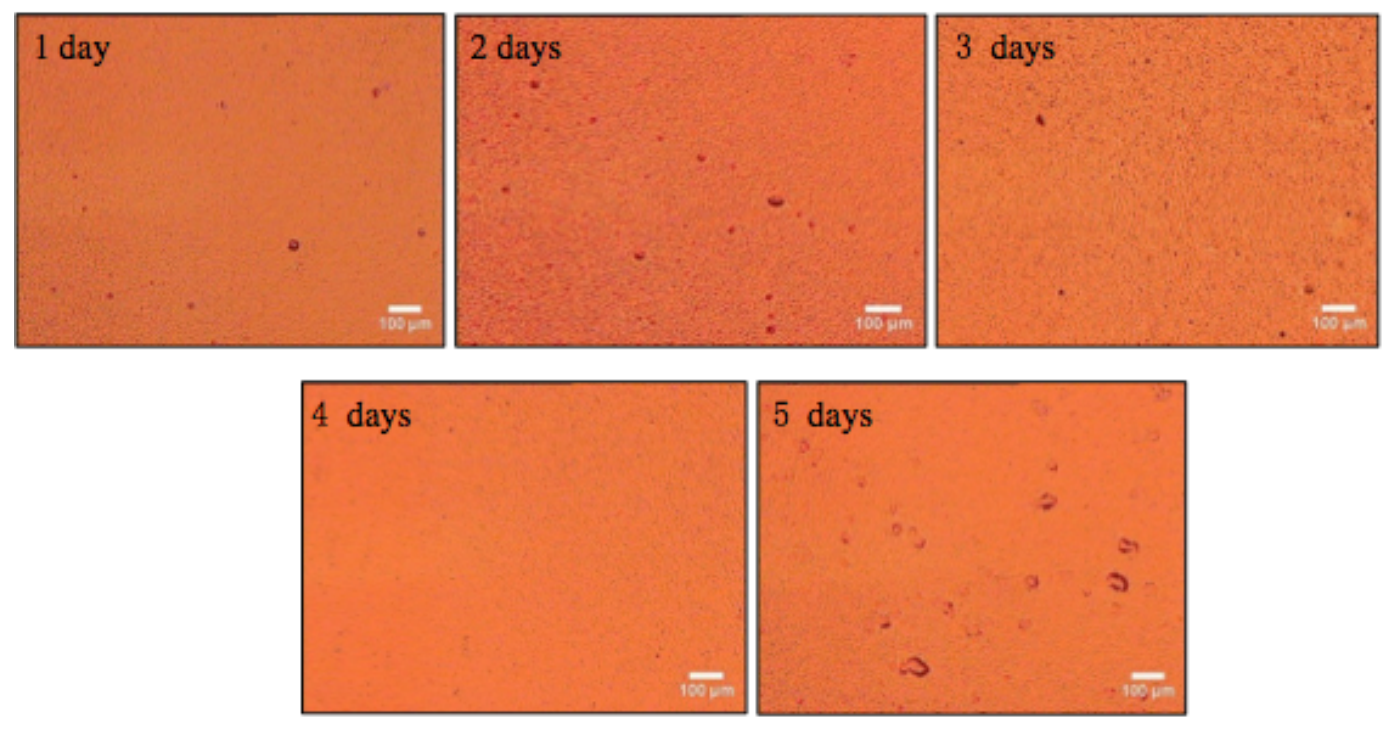

Figure 2.22. Optical microscope images of the surface topography of $150 \mu \mathrm{m}$-thick copper film deposited in a Cl-PEG-MPSA-PVP additive system of different aging time.

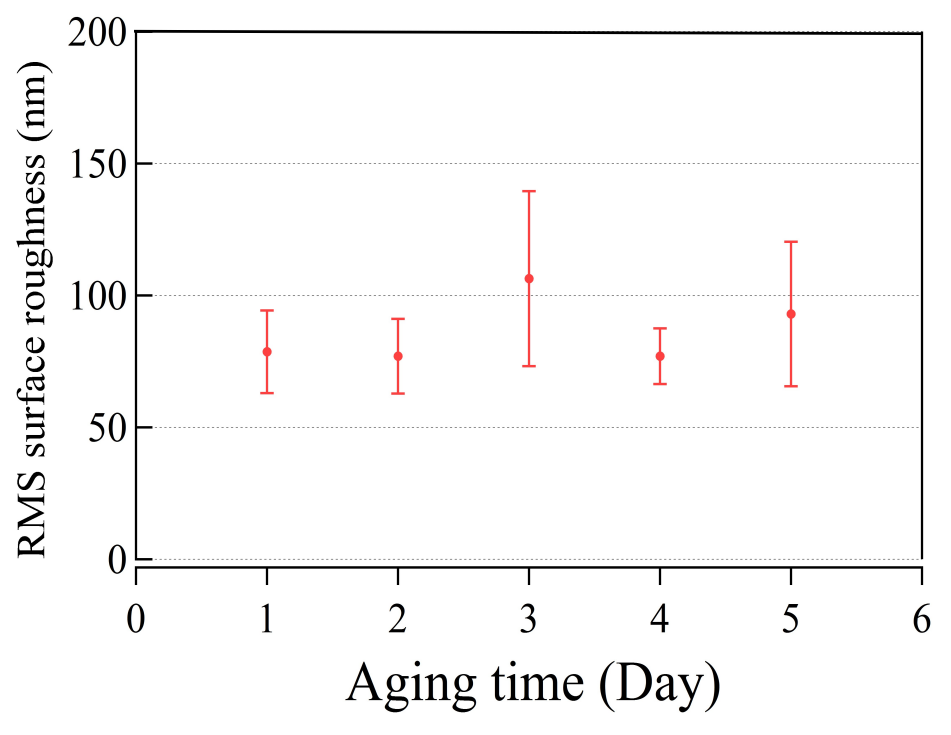

Figure 2.23. RMS surface roughness data of the $150 \mu \mathrm{m}$-thick copper film deposited in Cl-PEG-MPSA-PVP additive system of different aging time. Five different positions on each sample were measured by an optical profilometer. 
an aged electrolyte with a controlled current density of $10 \mathrm{~mA} / \mathrm{cm}^{2}$. As shown in Fig. 2.22, all the electrodeposited copper films are bright and only have a few defects. Moreover, Fig. 2.23 shows relatively similar RMS surface roughness data in all the samples with a range from 60 to $100 \mathrm{~nm}$. Both the optical microscope images and the surface roughness data indicate that the aging of the electrolyte does not have a significant impact on the surface roughness of electrodeposited copper films.

As is known, the resistivity of bulk copper is $1.7 \mu \Omega \mathrm{cm}$ [48] and the resistivity range of the deposited copper film ranges from 3.0 to $3.4 \mu \Omega \mathrm{cm}$ immediately after the electrodeposition as shown in Fig. 2.24. During the aging of the copper films, a $29 \%$ decrease in resistivity appears gradually. The resistivity drop is due to recrystalization and the growth of grains which resulted in a reduction of the grain boundary density [21]. In addition, there is a trend that the copper resistivity increases with bath aging. Also, the resistivity of copper electrodeposited from the electrolyte that was aged 3-4 days increases rapidly after 7 days of environmental exposure. Other work $[19,53]$ has shown that additives in the electrolyte tend to form $\mathrm{Cu}(\mathrm{I})$-accelerator and $\mathrm{Cu}(\mathrm{I})$-Cl-PEG complexes during aging that lead to decreased additive concentration. Therefore, as the electrolyte ages, defect structures increase causing the resistivity to rise in the electrodeposited film.

Fig. 2.25 shows the cross-section of metamaterial electrodeposited in aged electrolyte. There are no voids or seams forming in the trenches, which means all the aged electrolytes give rise to the superfilling of electrodeposition. However, overplating appears above the superfilled area that is due to the accumulation of the accelerators. Fig. 2.26 shows the cyclic voltammogram of fresh and aged MPSA in acid-copper solution. As the aging time increases, the maximum current of the reduction reaction also increases. It indicates that there is a change of the electrolyte during aging, either in composition or concentration, which may be caused by the degradation of MPSA. As other work stated, in a Cl-MPSA-PEG-PVP additive system, MPSA is the most 


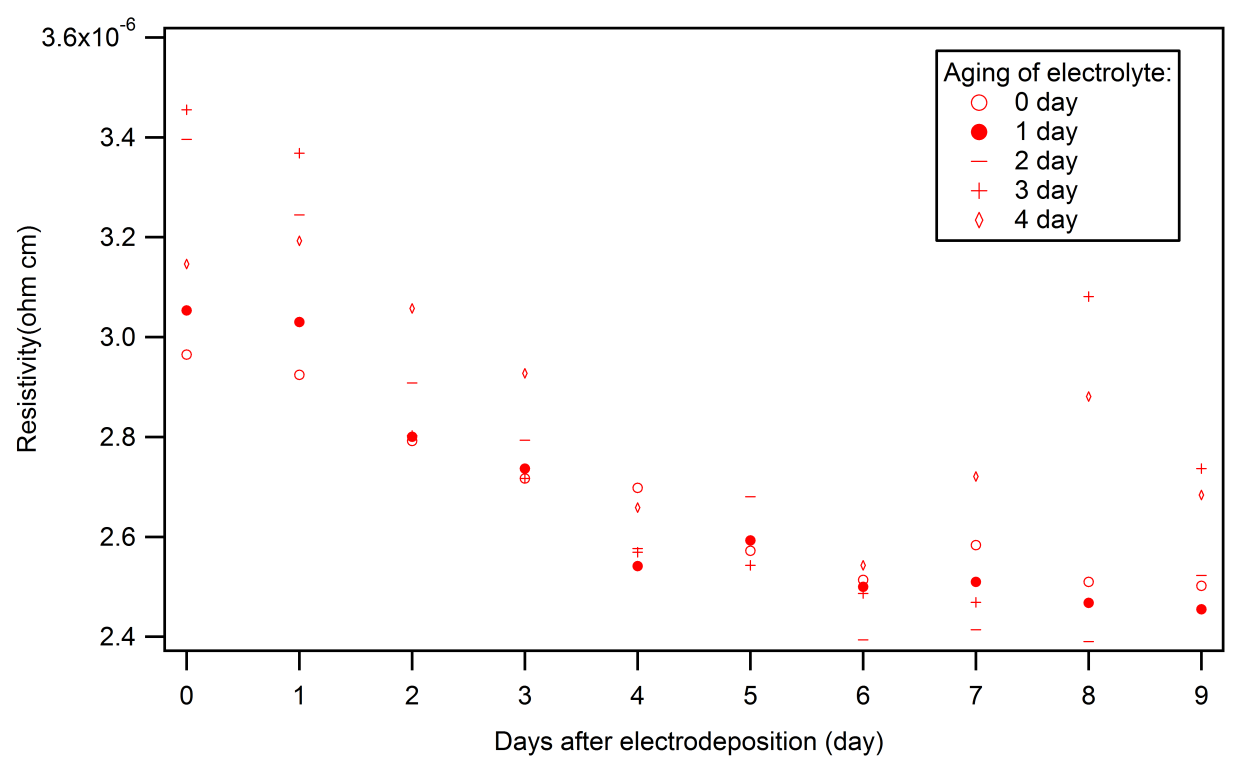

Figure 2.24. Resistivity transients during aging of $150 \mu \mathrm{m}$-thick copper film electrodeposited in aged electrolyte.
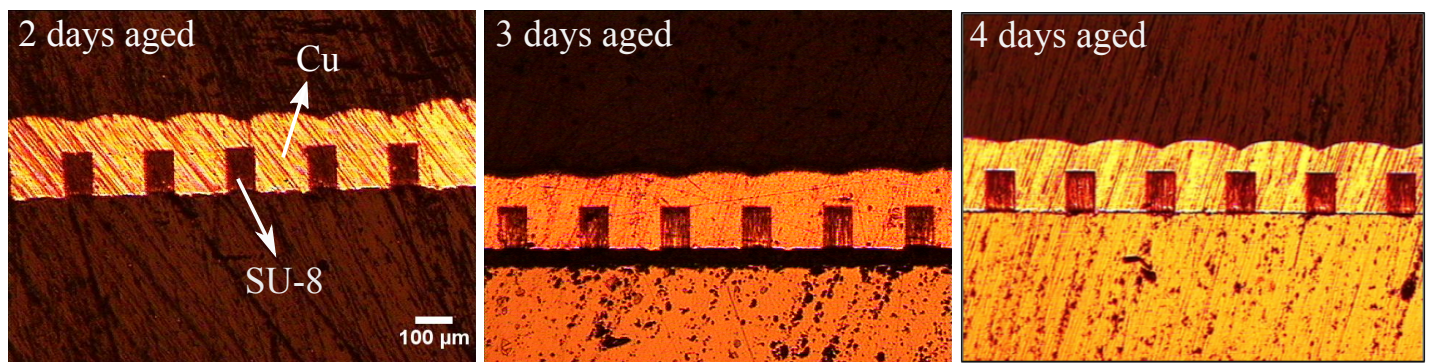

Figure 2.25. Cross-section of MMs that electrodeposited in aged electrolyte. 


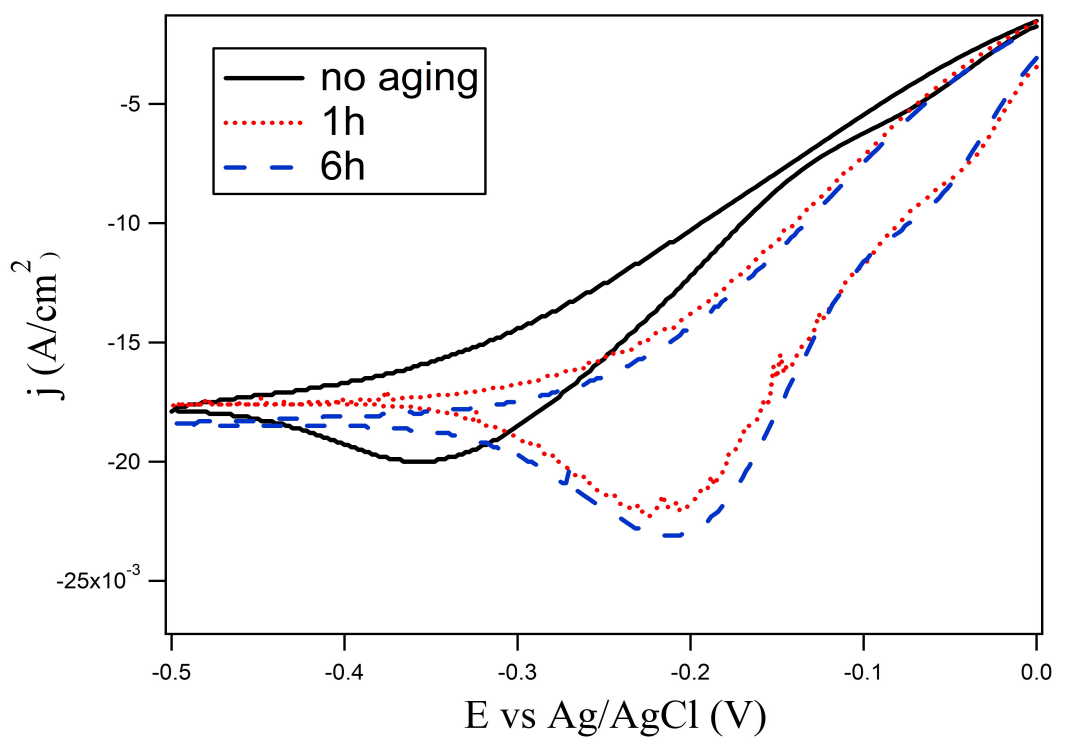

Figure 2.26. Cyclic voltammograms of fresh MPSA and MPSA aged for $1 \mathrm{~h}$ and $6 \mathrm{~h}$ in acid copper. All the electrodes were in the acid copper solution during all the measurements. There was no current flow during the aging process.

unstable component since in the presence of $\mathrm{Cu}^{2+}$, thiols $(\mathrm{S}-\mathrm{H})$ can be easily oxidized resulting in the formation of disulfides and this process is reversible $[19,41]$. In the present studies, ${ }^{1} \mathrm{H}-\mathrm{NMR}$ was used to characterize the degradation and the concentration change of the additives. As shown in the chemical structures in Fig. 2.27, both MPSA and SPS have three different kinds of protons, with the only difference that SPS is a dimer of MPSA. As shown in Fig. 2.27(a), the spectra of MPSA aged in the NMR solvent $\mathrm{D}_{2} \mathrm{O}$ for one hour, two days and three days are the same as that of fresh MPSA, having three peaks at $2.07 \mathrm{ppm}$ (M1), $2.71 \mathrm{ppm}$ (M2), and $3.07 \mathrm{ppm}$ (M3). The identical ${ }^{1} \mathrm{H}-\mathrm{NMR}$ spectra indicates that MPSA is stable at ambient conditions in water.

Fig. 2.27(b) is the ${ }^{1} \mathrm{H}-\mathrm{NMR}$ spectra of the powder extracted from fresh and aged solutions consisting of MPSA and acid-copper bath. It is apparent that all four spectra are the same, which means the compound in the solution remains the same in 


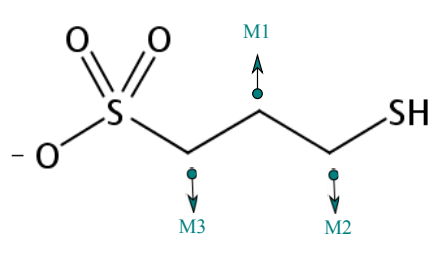

MPSA

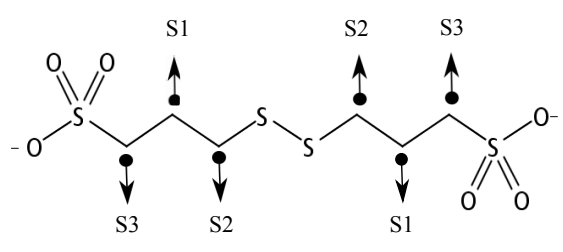

SPS

M3 M2

(a)
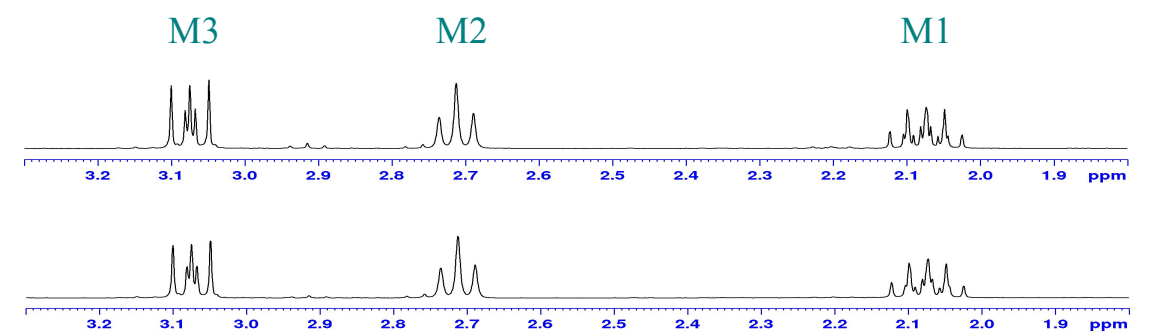

$1 \mathrm{~h}$

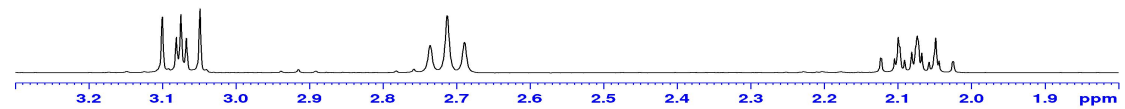

2 day

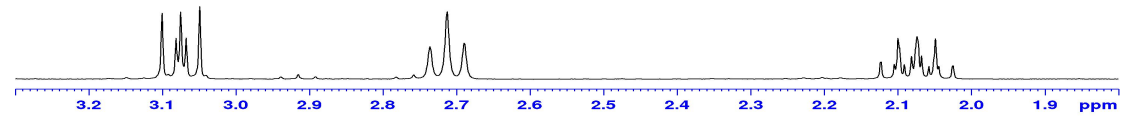

3 day

Oh

2

S3 S2

S1

(b)

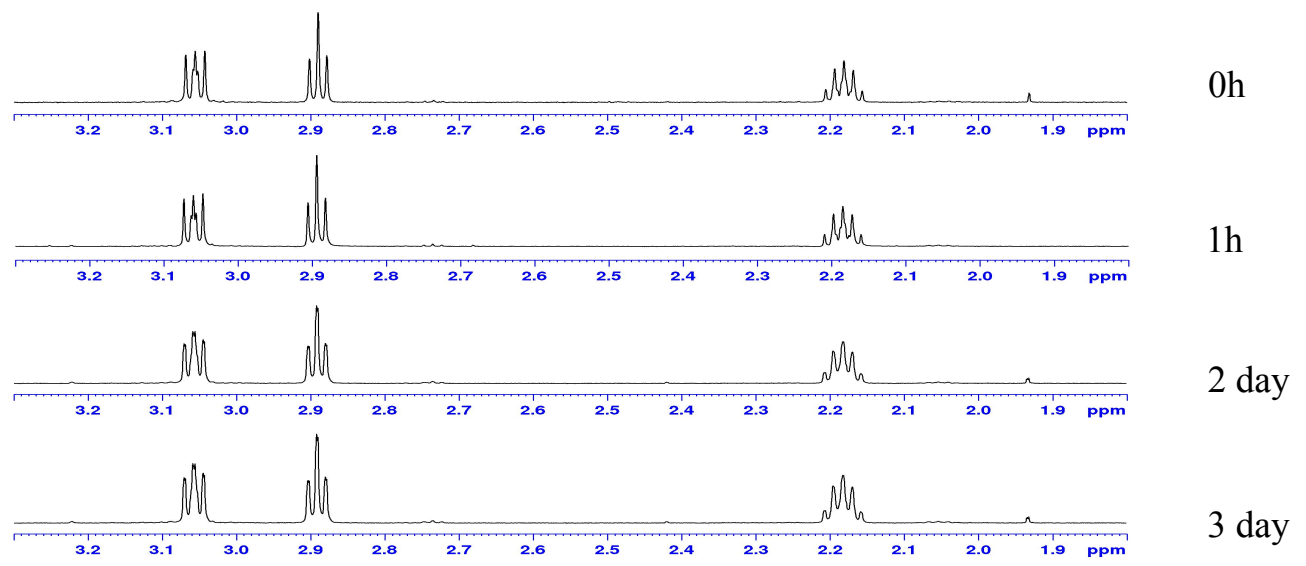

Chemical shift

Figure 2.27. ${ }^{1} \mathrm{H}-\mathrm{NMR}$ spectra of MPSA aged in water and MPSA aged in acid copper solution. 
both fresh and aged solutions. Besides, in Fig. 2.27(b), the chemical shift of the peaks S1 (2.18 ppm), S2 (2.89 ppm), and S3 (3.06 ppm) agree with the three characteristic peaks of pure SPS that can be found in the work of Choe et al. [41], indicating that MPSA converts to SPS in acid copper solution immediately and that SPS does not convert back to MPSA during aging. In contrast with others' work, the present data show that this conversion process is not reversible, for which two potential causes are likely. First, it might be caused by different experimental conditions such as low $\mathrm{pH}$ and the concentration of copper ions. Second, it should be noted that the extraction process of MPSA in acid copper bath involves neutralization, extraction, and evaporation. Among those three steps, neutralization is an exothermic process, and evaporation is performed under vacuum and high temperature $\left(55^{\circ} \mathrm{C}\right)$, so overheating might change both the original and regenerated MPSA to SPS, resulting in the appearance of only SPS in the spectra as shown in Fig 2.27(b). In order to investigate the impact of the pretreatment on the conversion of compounds, the following tests were conducted: pure MPSA powder was dissolved in DI water first and then evaporated under the same evaporation conditions as before whose ${ }^{1} \mathrm{H}-\mathrm{NMR}$ spectrum can be seen in Fig. 2.28(b) and the FTIR spectrum can be seen in Fig. 2.28 (right). The characteristic peaks of the ${ }^{1} \mathrm{H}-\mathrm{NMR}$ spectrum are the same as that of pure MPSA with an area ratio of 1:1:1. In the FTIR image, both the fresh and evaporated MPSA have the same spectra, especially the weak peak of S-H at $2550 \mathrm{~cm}^{-1}$, which proves again after the evaporation treatment, MPSA would still remain unchanged. The acid copper bath is likely the reason to drive MPSA to SPS, and this process is irreversible under the present experimental conditions.

Fig. 2.29 shows cyclic voltammograms of the electrolyte consisting of MPSA-ClPEG-PVP additives in acid copper solution used for electrodeposition for different lengths of time. It is clear that the magnitude of the hysteresis decreases with an increase in the electrodeposition time. The decrease in the forward current density 
(a)

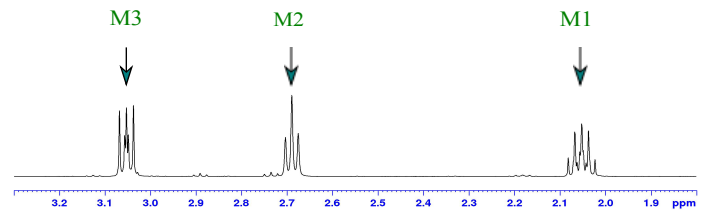

(b)
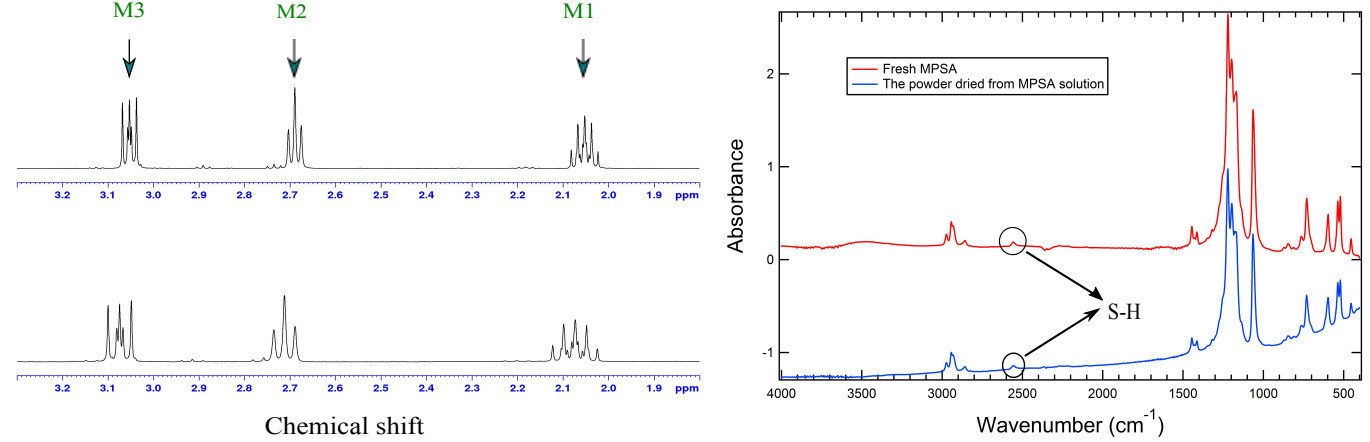

Figure 2.28. ${ }^{1} \mathrm{H}-\mathrm{NMR}$ spectra (left) and the FTIR spectra (right) of (a) fresh MPSA and (b) the powder dried from MPSA + DI water solution.

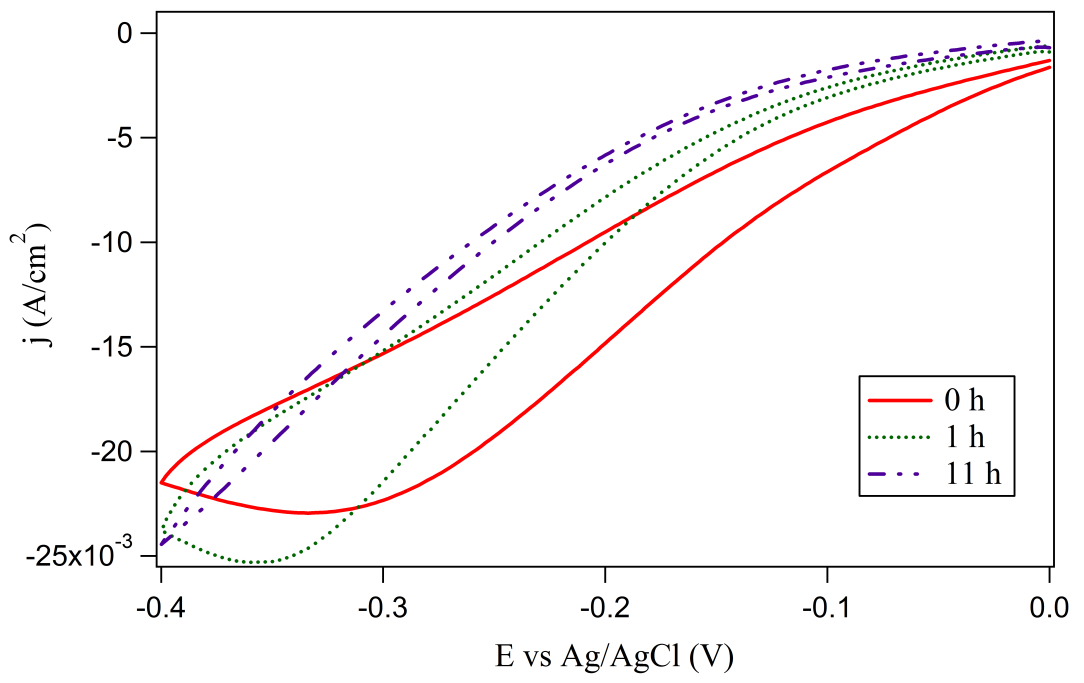

Figure 2.29. Cyclic voltammogram of fresh electrolyte and electrolytes used for electrodeposition for $1 \mathrm{~h}$ and $11 \mathrm{~h}$. The electrolyte consists of acid copper and PEG-MPSA-Cl-PVP four additives. 
(a)

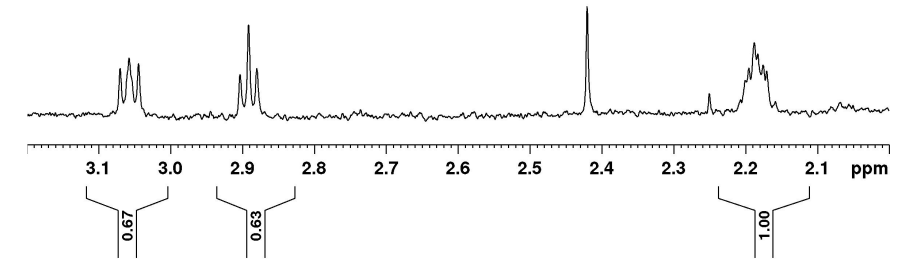

(b)

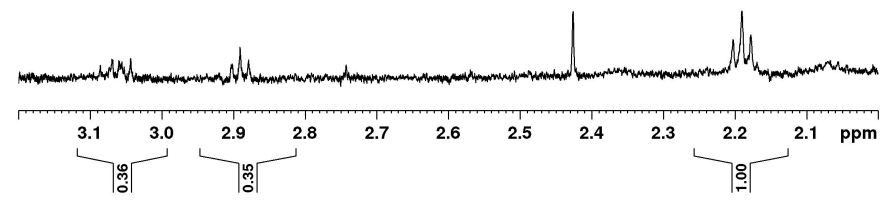

Chemical shift

Figure 2.30. ${ }^{1} \mathrm{H}-\mathrm{NMR}$ spectra of (a) the powder extracted from unused electrolyte and (b) the powder extracted from used electrolyte. The electrolyte consists of acid copper and PEG-MPSA-Cl-PVP four additives.

and the disappearance of the hysteresis means that with current flow the effect of the additives decreases with time.

As shown in Fig. 2.30, the chemical shift of the three characteristic peaks of the accelerator is the same as that of SPS (2.18 ppm, $2.89 \mathrm{ppm}, 3.06 \mathrm{ppm})$ but the ratios of the three peaks are 1:0.63:0.67 for the fresh electrolyte and 1:0.35:0.36 for the used electrolyte, indicating degradation of the accelerators over time. It is conceivable that in the presence of three more additives, more interactions take place between additives, especially since the accelerators form complexes with copper ions and other additives, which has also been shown by mass spectrometry conducted by other researchers $[53,54]$. Additionally, there is a single peak at $2.4 \mathrm{ppm}$, which aligns with the characteristic peak of H-C-S, indicating MPSA and SPS would break down into segments during aging and electrodeposition. 


\subsubsection{Terahertz transmission measurement}

A series of transmission experiments for metamaterials were carried out with terahertz time-domain spectrometer in transmission mode. The radiation generated from the system ranges from 0.4 to $5 \mathrm{THz}$. For each transmission measurement, the electric field magnitude vs. time domain spectrum is recorded, then the frequency spectrum can be obtained by Fourier transform.

Fig. 2.31 and Fig. 2.32 are the transmission spectra to metamaterials with different thicknesses of copper grown on the substrates with $150 \mu \mathrm{m}$-tall pillars and $40 \mu \mathrm{m}$ tall pillars, respectively. Compared to Fig. 2.32, Fig. 2.31 shows a series of sharper resonance peaks, which indicates that compared to $40 \mu \mathrm{m}$ pillars, $150 \mu \mathrm{m}$ pillars provide enhanced resonance to MMs at $1 \mathrm{THz}$.

Also, as shown in Fig. 2.31, the transmission intensity decreases with an increase of the copper thickness, and a surface plasmon resonance appears around $1 \mathrm{THz}$. For the metamaterial with $100 \mathrm{~nm}$ thick copper, no metamaterial shift was observed at $0.5 \mathrm{THz}$, where surface plasmon resonance takes place. As the copper thickness increases from $100 \mathrm{~nm}$ to $150 \mu \mathrm{m}$, surface plasmon appears at a higher radiation frequency, from $0.5 \mathrm{THz}$ to $1.2 \mathrm{THz}$; while the maximum transmission drops from $100 \%$ to $17 \%$. The appearance of transmission peaks indicates that the geometry of metamaterial, especially the pillars structure, has a significant correlation to surface plasmon resonance.

Besides, in Fig. 2.31, the transmission completely disappears when the copper layer is $200 \mu \mathrm{m}$ thick. As is common knowledge, an irradiated objects can either absorb, reflect or transmit the radiation in some combination. If the transmitted portion is zero, the quantity absorbed can be easily obtained by just measuring the reflection spectrum, which simplifies the optical characterization and the geometry design of metamaterials. Comparing all of the different geometric parameters, there are three potential reasons for zero transmission: overplating, metamaterial thickness, and the 


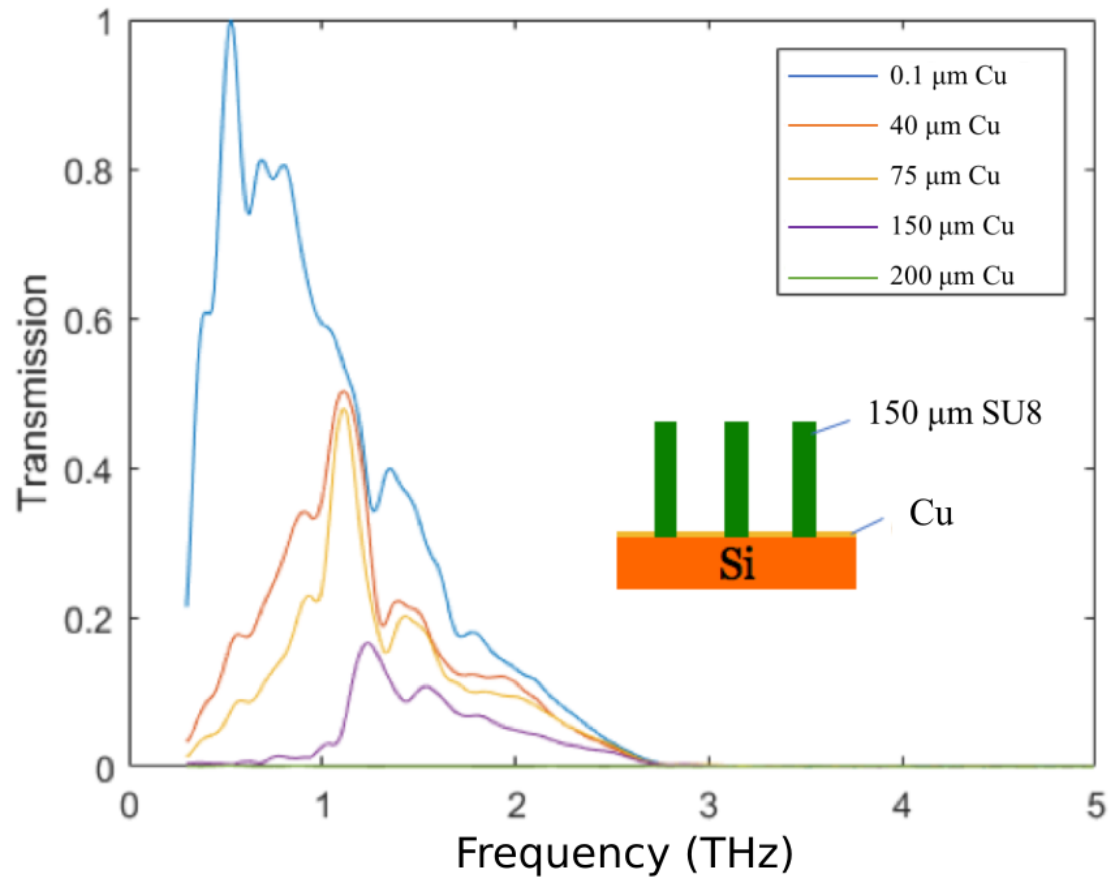

Figure 2.31. Terahertz transmission to MMs with $150 \mu \mathrm{m}$-tall SU-8 pillars and different thickness of copper.

metal/dielectric height ratio. In order to investigate the cause of zero transmission, two overplated metamaterials were manufactured to compare their tramsmission spectra as shown in Fig. 2.33. It is clear that the overplated metamaterials with $40 \mu \mathrm{m}$ thick pillar does not give rise to zero transmission, indicating that overplating is not the cause of zero transmission. Fig. 2.34, 2.35, and 2.36 show the transmission spectra of MMs with different metal/dielectric height ratios ranging from 0 to 1 . It is apparent that all the metamaterials can transmit terahertz radiation, indicating that the metal/dielectric height ratio is not the reason for zero transmission. After ruling out the cause of overplating and metal/dielectric height ratio, we can conclude from Figs. 2.33-2.36 that the transmission has a strong correlation with the thickness of metamaterial. In our study, this transmission disappears when the metamaterial consists of $150 \mu \mathrm{m}$ thick pillars and $200 \mu \mathrm{m}$ thick copper. 


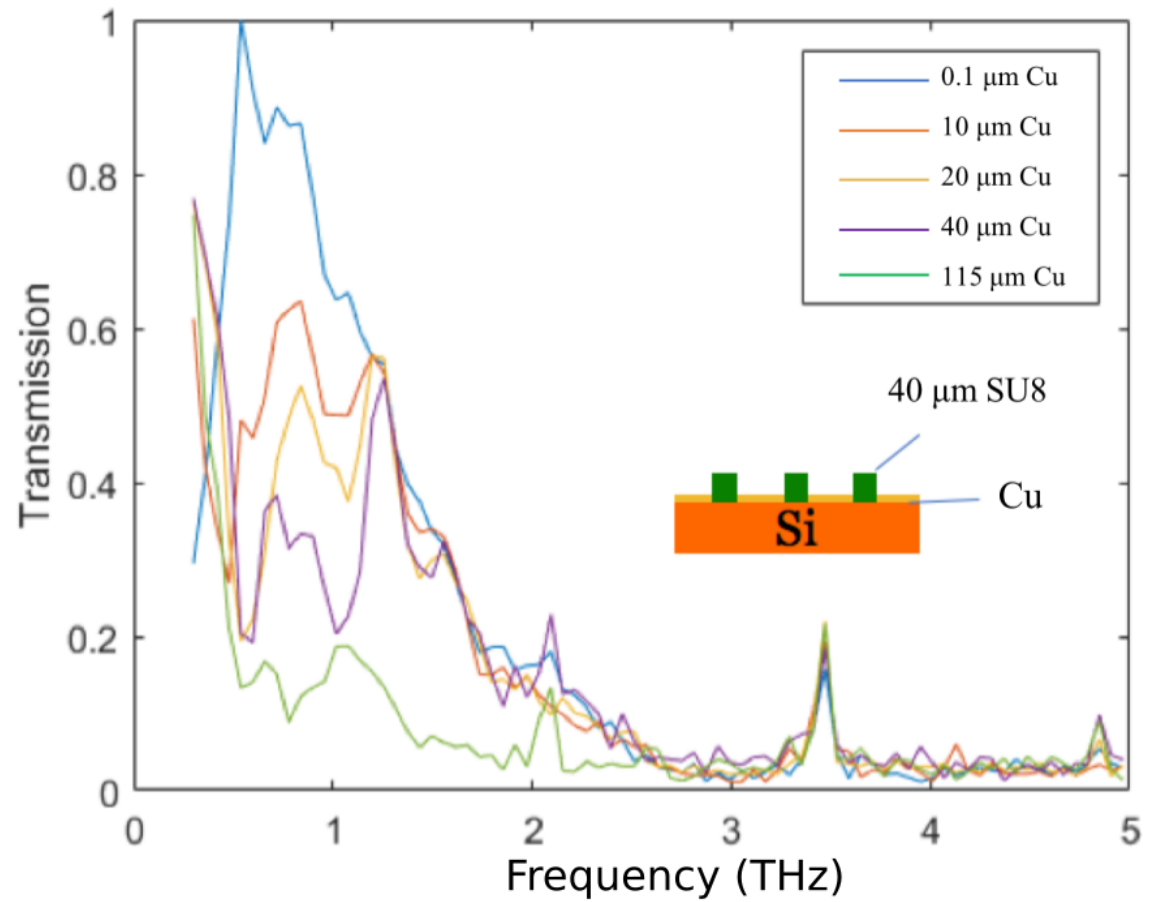

Figure 2.32. Terahertz transmission to MMs with $40 \mu \mathrm{m}$-tall SU-8 pillars and different thickness of copper.

As shown in Fig. 2.34, the transmission performance of MMs with $100 \mathrm{~nm}$ thick copper and different pillar heights is very similar in that both of them have a significant transmission spectrum at around $1 \mathrm{THz}$, and that there appears 100\% transmission at $0.7 \mathrm{THz}$. It means that MM pillars have the ability to manipulate terahertz radiation.

As shown in Fig. 2.35, the metamaterial with $150 \mu \mathrm{m}$ pillars and $75 \mu \mathrm{m}$ copper both gives rise to a sharp transmission spectrum, showing a surface plasmon resonance at $1 \mathrm{THz}$ with a bandwidth of $\pm 0.1 \mathrm{THz}$. However, there appears a double transmission peak at $1 \pm 0.4 \mathrm{THz}$ when the $\mathrm{THz}$ radiation irradiates on the metamaterial with $40 \mu \mathrm{m}$ pillars and $20 \mu \mathrm{m}$ copper. So it indicates that, compared to the metamaterial with $40 \mu \mathrm{m}$ pillars and $20 \mu \mathrm{m}$ copper, $150 \mu \mathrm{m}$ pillars and $75 \mu \mathrm{m}$ copper 

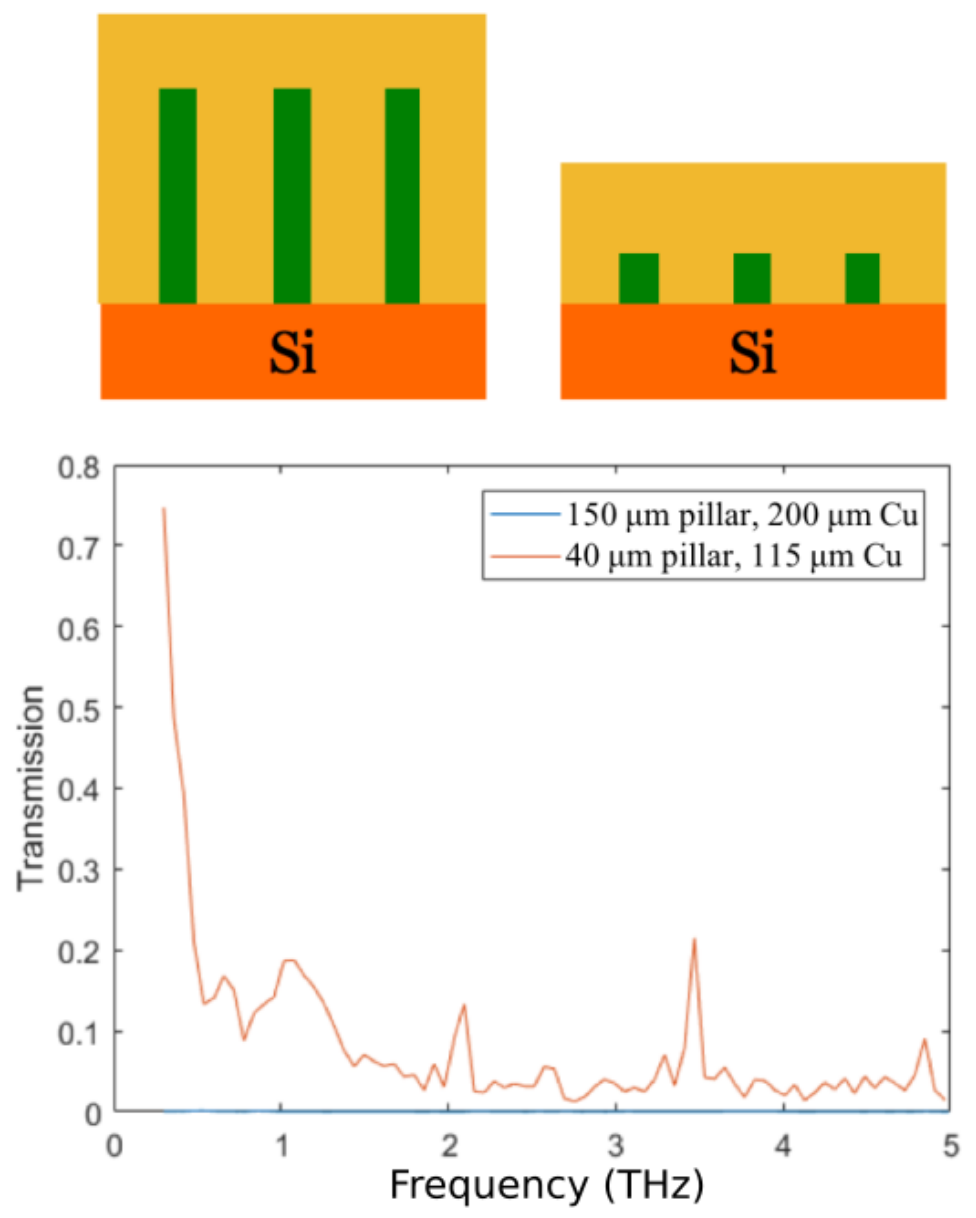

Figure 2.33. Comparison of transmission for MMs with overplated copper. 

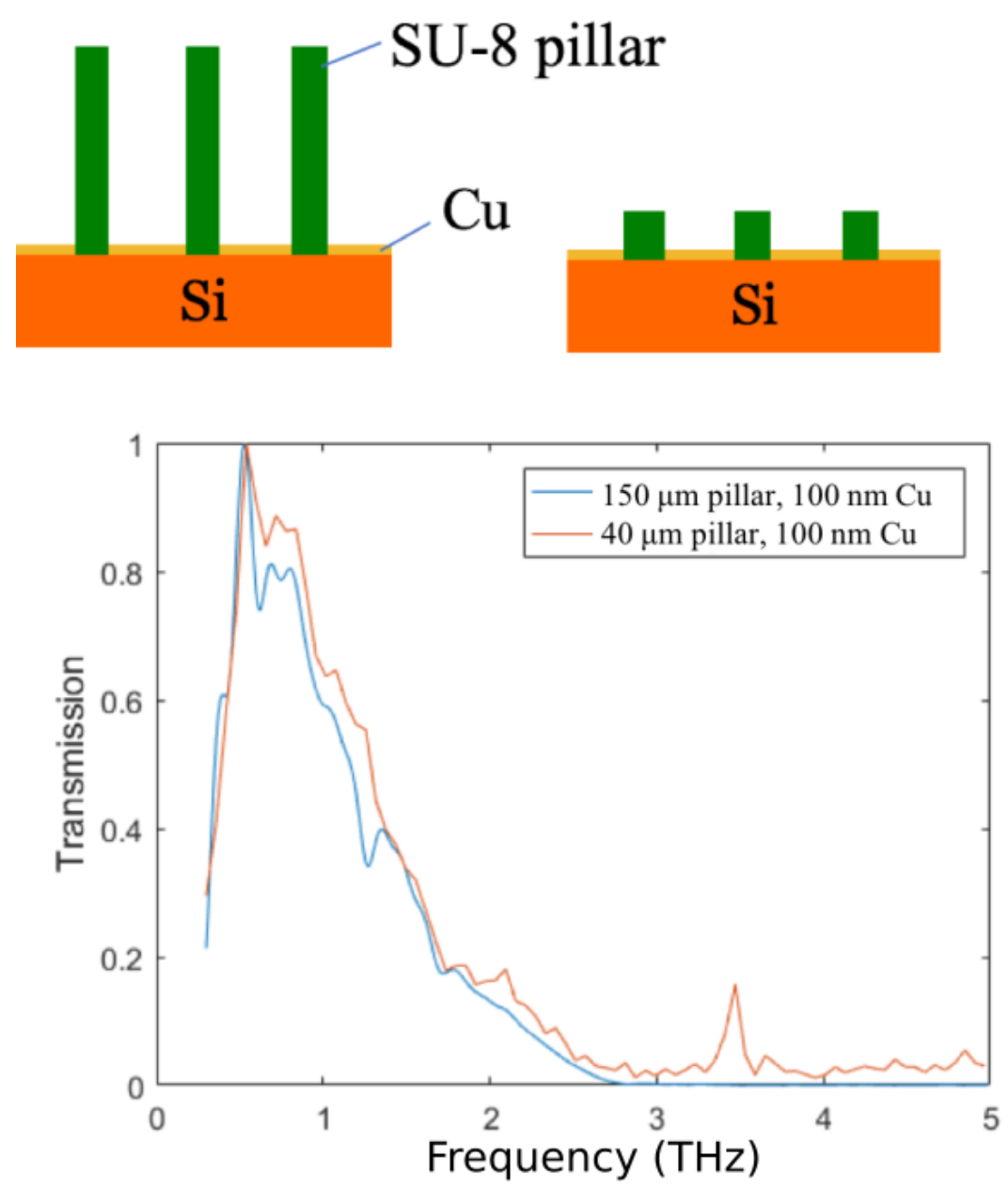

Figure 2.34. Comparison of transmission for MMs with $100 \mathrm{~nm}$ thick copper and different thicknesses of SU-8 pillars. The metal/dielectric height ratio is approximately equal to 0. 

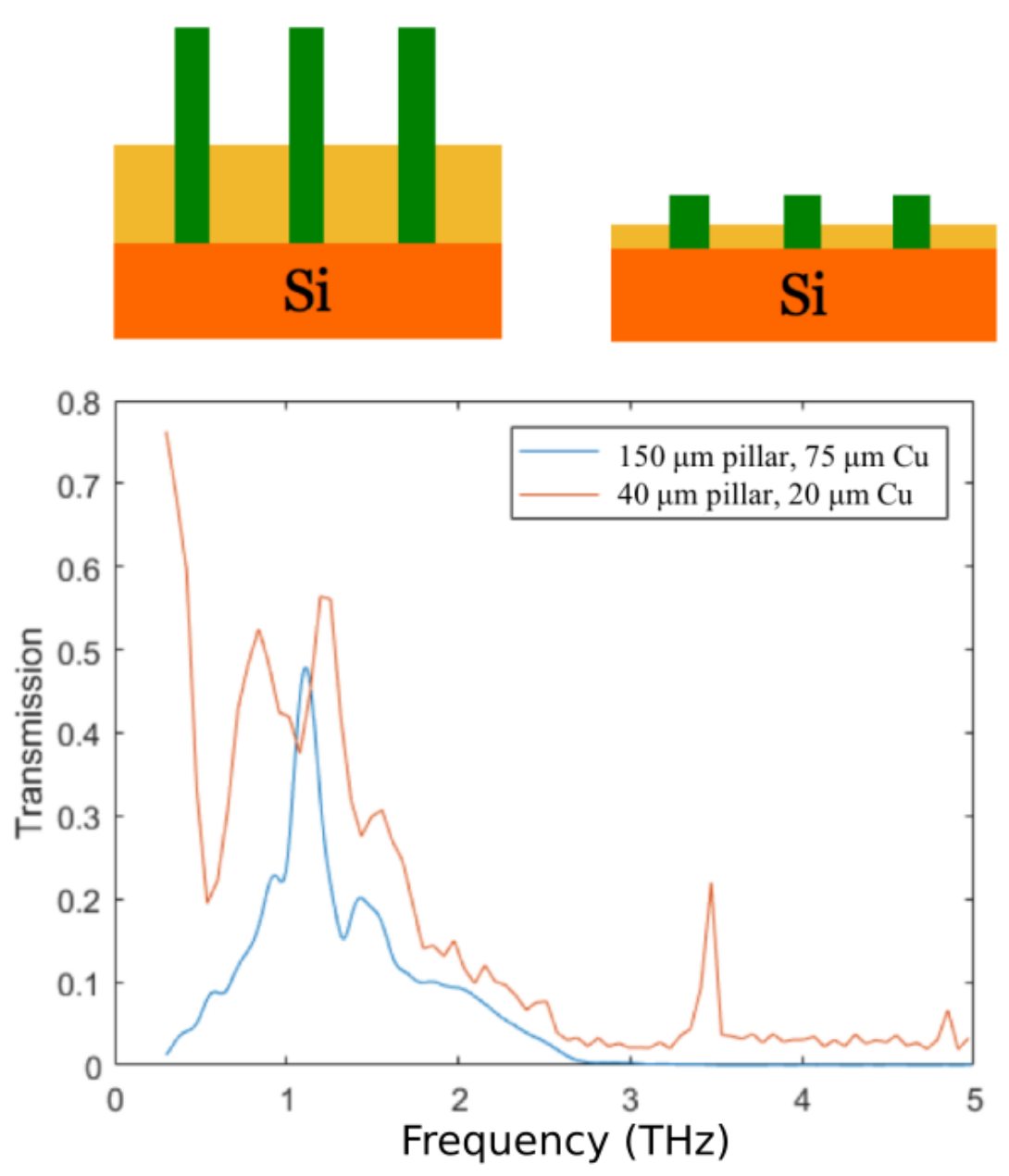

Figure 2.35. Comparison of transmission for MMs with halfway-covered copper. The metal/dielectric height ratio is 0.5 .

can couple $\mathrm{THz}$ radiation to the propagating plasmon and generate surface plasmon resonance at $1 \mathrm{THz}$ more efficiently.

Figure 2.36 shows the comparison of transmission for metamaterials with same metal/dielectric heights. In general, the metamaterial with $150 \mu \mathrm{m}$ pillar and copper has a weaker transmission spectrum and less noise when compared to metamaterial with $40 \mu \mathrm{m}$ pillar and copper. Besides, the maximum transmission of $150 \mu \mathrm{m}$ metamaterial is $16 \%$ while that of $40 \mu \mathrm{m}$ is $52 \%$. Figure 2.36 indicates that with the same metal/dielectric height, thicker metamaterial give rise to a more coherent sur- 

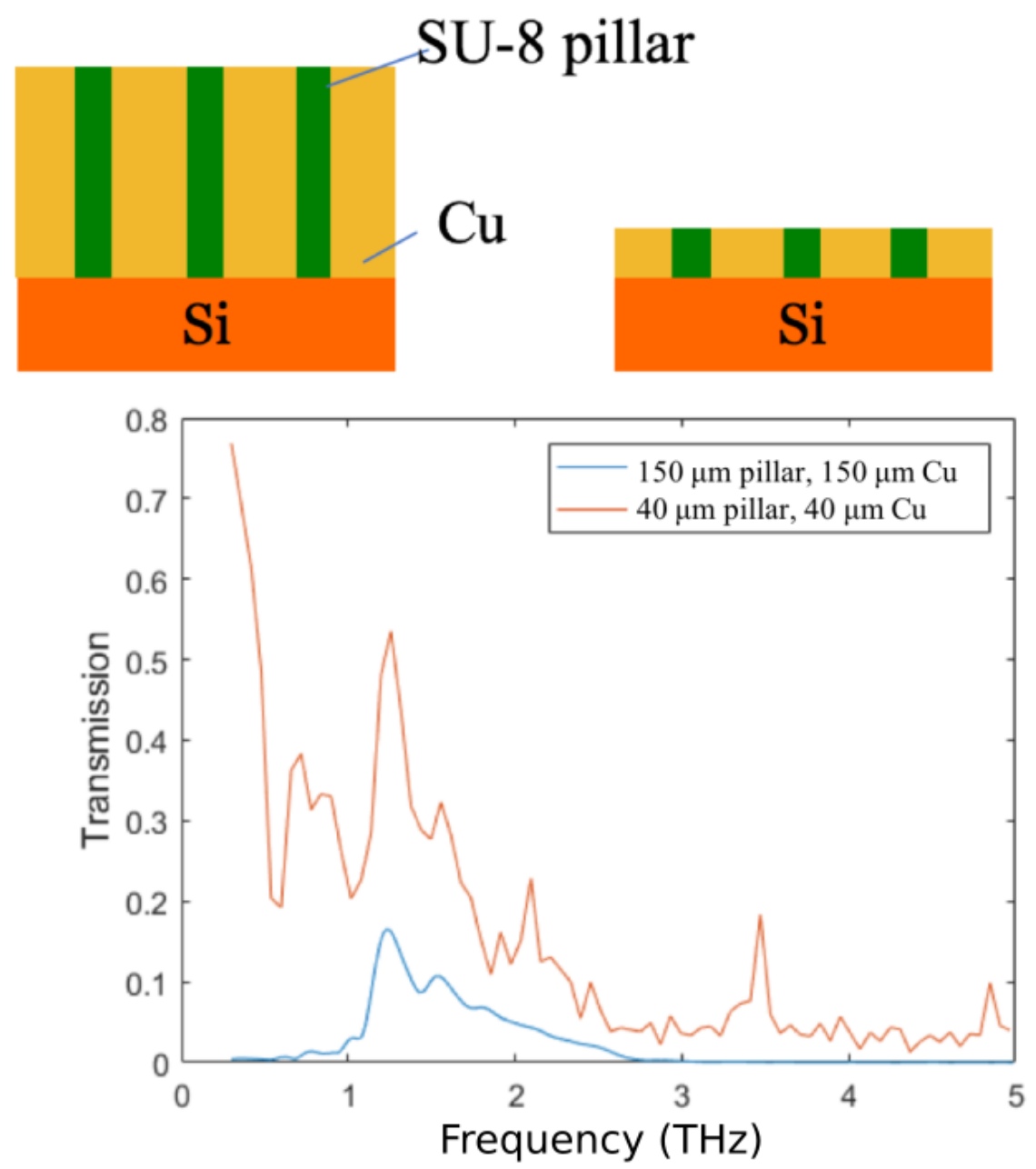

Figure 2.36. Comparison of transmission for MMs with fully-covered copper. The metal/dielectric height ratio is 1 . 
face plasmon and less noise. Also, it proves again that thicker metamaterial causes a decrease in the transmission magnitude.

\subsection{Conclusions}

A series of experiments were conducted involving the electrodeposition of copper for the fabrication of metamaterials under different conditions. In our work, ClMPSA-PEG-PVP additives can interplay with each other well, and they are effective in both bottom-up superfill and leveling for electrodeposition of copper for metamaterials. Compared to PVP, another leveler, Janus Green B, exhibits a better leveling effect. NMR and FTIR data show that even without current flow, the accelerator MPSA converts to SPS in an acid copper bath immediately, and this process is not reversible in our experimental conditions. When there are Cl-MPSA-PEG-PVP four additives in the acid copper solution, accelerators interact with other additives and form complexes resulting in the breakdown of accelerators and the decrease of the concentration; with current flow, this degradation process can be enhanced.

We have also successfully conducted a series of 2D COMSOL simulations of electrodeposition and investigated the mechanism of basic electrodeposition, the superfilling of features, and the adsorption of accelerators. The COMSOL simulation shows that additives are very important to get uniform and bright deposits, and it illustrates that the accelerators accumulate and move vertically in the trench first; after overplating happens, accelerators move along the lateral direction.

The transmission experiments show that the geometry of metamaterial, especially the pillars structure, has a significant correlation to surface plasmon resonance. We can also conclude that the transmission has a strong correlation with the thickness of metamaterial. Compared to the metamaterial with $40 \mu \mathrm{m}$ pillars and $20 \mu \mathrm{m}$ copper, $150 \mu \mathrm{m}$ pillars and $75 \mu \mathrm{m}$ copper can couple $\mathrm{THz}$ radiation to the propagating plasmon and generate surface plasmon resonance at $1 \mathrm{THz}$ more efficiently. 


\section{CHAPTER 3}

\section{OPTIMIZATION OF THREE-DIMENSIONAL METAMATERIAL: ELECTROMAGNETIC SIMULATION}

\subsection{Introduction}

The previous chapter discussed how metamaterials were made, this chapter will demonstrate the finite element method (FEM) simulation of metamaterials on the basis of its electromagnetic properties. Three-dimensional transmission and reflection simulations for metamaterials were investigated with COMSOL multiphysics software using radio frequency $(\mathrm{RF})$ module.

\subsubsection{FEM simulation}

Finite element method (FEM) is a numerical method for solving problems of engineering and mathematical physics, which originated from the need to solve complex elasticity and structural analysis problems in civil and aeronautical engineering in the 1940s. The basic concept in the physical interpretation of the FEM is the subdivision of the mathematical model into disjoint components of simple geometry called finite elements [55].

Generally, most of engineering problems occur in nature involves partial differential equations (PDE). FEM is a method to solve PDEs by converting them into

algebraic equations or rewriting them as approximations of ordinary differential equations (ODE). Thus, these equations can be solved using standard numerical techniques such as Euler and Newton method. 
In FEM, the domain of the simulation structure is subdivided into a collection of sub-domain elements via meshing. The original problem of each element can be calculated by a set of element equations constrained by sets of initial conditions and boundary conditions. Then all the element equations can be recombined into a global system of equations for final calculation. FEM uses variational methods from the calculus of variations to approximate a solution by minimizing an associated error function. The approximations are usually polynomial and interpolations over the element. Only certain points within the elements are known, which are so called nodal points. And the majority of those points are located at the boundary of the element.

There are around fifty FEM software packages, such as COMSOL multiphysics, ANSYS Mechanical, Abaqus/CAE, etc. COMSOL multiphysics, one of the most widely used FEM software, is an integrated environment for solving systems of timedependent or stationary second order in space partial differential equations in one, two, and three dimensions. COMSOL multiphysics has a few application modules, including electrical, mechanical, fluid, chemical, multipurpose, and interfacing modules. COMSOL multiphysics easily integrates with MATLAB to extend modeling with script programming. It can link also with SolidWorks to enhance the simulation into design workflow, and it is also compatible with CAD to create geometries for simulation.

\subsubsection{Transmission and reflection theory}

In this project, it is important to learn how metamaterials respond to EM waves in its designed operation range. As is known, there are three types of interactions between incident radiation and irradiated object: transmission $(\mathrm{T})$, reflection $(\mathrm{R})$ and absorption (A). The summation of those three portions is unity:

$$
T+R+A=1
$$


Since absorption is hard to quantify, it can be calculated after knowing the transmission and reflection. All transmission and reflection simulations are based on Maxwell's equation and its alternate forms.

\subsubsection{Relative permittivity and refractive index}

In FEM simulation, some frequency-dependent variables for the refractive index in terahertz range are required for material and geometry selection of MMs. Permittivity, $\varepsilon$, is a one of the frequency-dependent material properties, which defined as the ability of a substance to store electric energy in an electric field. It controls the speed at which the electric radiation can propagate the fields, and also controls the amount of force the two charges will feel due to the interaction force between them. The relative

permittivity (dielectric constant) of a material is its absolute permittivity expressed as a ratio relative to the permittivity of vacuum. The refractive index of a material is a dimensionless number that describes how light propagates through a medium. It is defined as

$$
n=\frac{c_{\circ}}{v}
$$

where $c_{\circ}$ is the speed of light in vacuum and $v$ is the phase velocity of light in the medium.

The refractive index is typically expressed as a complex quantity:

$$
\tilde{n}=n+i k
$$

where $\tilde{n}$ is the complex refractive index, $n$ is the real part of refractive index, and $k$, the extinction coefficient, represents the imaginary part of refractive index. Also, the complex relative permittivity is:

$$
\tilde{\varepsilon_{r}}=\varepsilon_{R}+i \varepsilon_{i}
$$


where $\tilde{\varepsilon_{r}}$ is the complex relative permittivity, $\varepsilon_{R}$ and $\varepsilon_{i}$ are the real part and the imaginary part of the complex relative permittivity.

Since the relationship between the complex relative permittivity $\tilde{\varepsilon_{r}}$ and the complex refractive index $\tilde{n}$ is represented as:

$$
\tilde{\varepsilon_{r}}=\tilde{n}^{2}
$$

thus,

$$
\begin{gathered}
\varepsilon_{R}+i \varepsilon_{i}=(n+i k)^{2} \\
\varepsilon_{R}=n^{2}-k^{2} \\
\varepsilon_{i}=2 n k
\end{gathered}
$$

Since $\varepsilon_{R}$ and $\varepsilon_{i}$ are material properties and can be obtained from literature (extropolation details shown in section 3.1.3). The refractive index $n$ and the extinction coefficient $k$ can be calculated through the derivation shown in Eqn. 3.6, 3.7 and 3.8.

\subsubsection{Plane wave}

A plane wave is a wave whose surfaces of constant phase are infinite planes, perpendicular to the direction of propagation [56]. Plane waves are commonly selected as a radiation source to simplify simulations. As is known, the electric field strength of wave is dependent on space and time, which can be written in the form:

$$
\boldsymbol{E}(x, y, z, t)=E(x, y, z) f(t)
$$

Thus,

$$
\boldsymbol{E}(x, y, z, t)=E(x, y, z) e^{i \omega t}
$$


where bold-faced $\boldsymbol{E}$ is the complete electric field, the lighter type $E$ is the timeindependent electric field and $\omega$ is the angular frequency of the wave. The function $E(x, y, z)$ accounts for the spatial dependence of the field, while $e^{i \omega t}$ represents the time variation. Since the vector wave equation [56] is expressed as:

$$
\nabla^{2} \boldsymbol{E}=\mu_{0} \varepsilon_{0} \varepsilon_{r} \frac{\partial^{2} \boldsymbol{E}}{\partial t^{2}}
$$

where $\mu_{0}$ is the vacuum permeability, $\varepsilon_{0}$ is the vacuum permittivity, $\varepsilon_{r}$ is the relative permittivity. So the time-independent vector wave equation is

$$
\nabla^{2} E=-\omega^{2} \mu_{0} \varepsilon_{0} \varepsilon_{r} E
$$

For plane waves (only Z direction):

$$
\frac{\mathrm{d}^{2} E(z)}{\mathrm{d} z^{2}}+\omega^{2} \mu_{0} \varepsilon_{0} \varepsilon_{r} E(z)=0
$$

Guess the solution of Eqn. 3.13 is:

$$
E(z)=E_{\circ} e^{(i K z)}
$$

where $E_{\circ}$ is the electric field magnitude. then substituting Eqn. 3.14 into Eqn. 3.13 and we obtain:

$$
K=\omega \sqrt{\mu_{0} \varepsilon_{0} \varepsilon_{r}}
$$

Because

$$
c_{\circ}=\frac{1}{\sqrt{\mu_{0} \varepsilon_{0}}},
$$


and the relationship between relative permittivity and refractive index shown in Eqn. 3.5 and 3.6, thus:

$$
K=\frac{\omega \sqrt{\varepsilon_{r}}}{c_{\circ}}=\frac{\omega(n+i k)}{c_{\circ}}
$$

The time-independent wave equation of plane wave propagating in $z$ direction is shown in Eqn. 3.18:

$$
E(z)=E_{\circ} e^{\frac{i(n+i k) \omega}{C_{\circ}} z}
$$

Since the refractive index $n$ and the extinction coefficient $k$ are known, the wave equation of plane wave as shown in Eqn. 3.18 can be solved.

\subsubsection{S-parameter}

The $S$-parameter, scattering parameter, expresses circuit characteristics using the degree of scattering when an AC signal strikes an interface [57]. The scattering involves the radiation reflected back to the source and transmitted to other directions. The input and output ports of a circuit are numbered, for example, $S_{\mathrm{xy}}$ represents the $S$-parameter that is incident at port $y$ and detected at port $x$. As shown in Fig. 3.1, if $x=y$, the wave is purely a reflection; if $x \neq y$, the wave is transmission. Since the $S$-parameter is the square root of the electrical power, it is a dimensionless parameter. The spectroscopic transmission and reflection can be represented using $S$-parameters

and the square of the absolute value of $S$-parameter as shown in Eqn. 3.19 and 3.21.

$$
S_{11}=E_{1} / E_{0} \quad ; \quad S_{21}=E_{2} / E_{0}
$$

Since

$$
\begin{gathered}
P \propto E^{2} \\
\left|S_{11}\right|^{2}=\frac{P_{1}}{P_{0}} \quad ; \quad\left|S_{21}\right|^{2}=\frac{P_{2}}{P_{0}}
\end{gathered}
$$




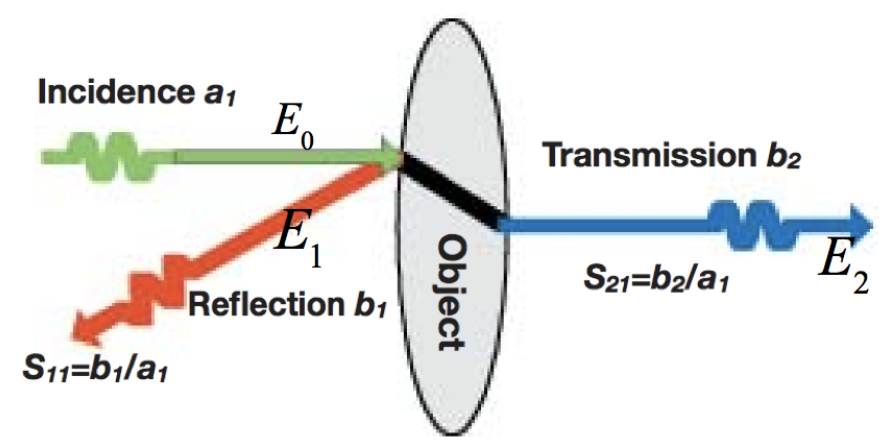

Figure 3.1. Conceptual diagram of $S$-parameter [57].

\subsubsection{Drude model}

The Drude model of electrical conductivity, proposed in 1990 by Paul Drude, demonstrates transport properties of electrons in materials (especially metals). It focuses on the description of basic tendencies of metal conductivity in terms of the frequency of electromagnetic field, crystal quality, temperature and alloying. The optical constants (refraction and extinction indexes) can be derived from the Drude model.

The Drude model is established on a few assumptions. First, metals are treated as a mass of positively charged ions with detached free electrons. Those free electrons are responsible for electrical conduction. Besides, the Drude model only considers the elastic collisions between electrons. After a collision, the kinetic energy of electron is only dependent on the local temperature distribution.

If we consider metal as a perfect conductor without defects and impurities then the electric field in the metal will be zero, in which case the dielectric constant will be infinity. As shown in Fig. 3.2, in the low frequency region (radio frequency to terahertz region), a metal's dielectric constant is a complex, with a very high imaginary part and a comparatively insignificant real part. As the field frequency increases, the dielectric constant becomes a negative value, meaning that metals reflect the electromagnetic wave in this frequency domain. As the frequency becomes even higher, the metal's 


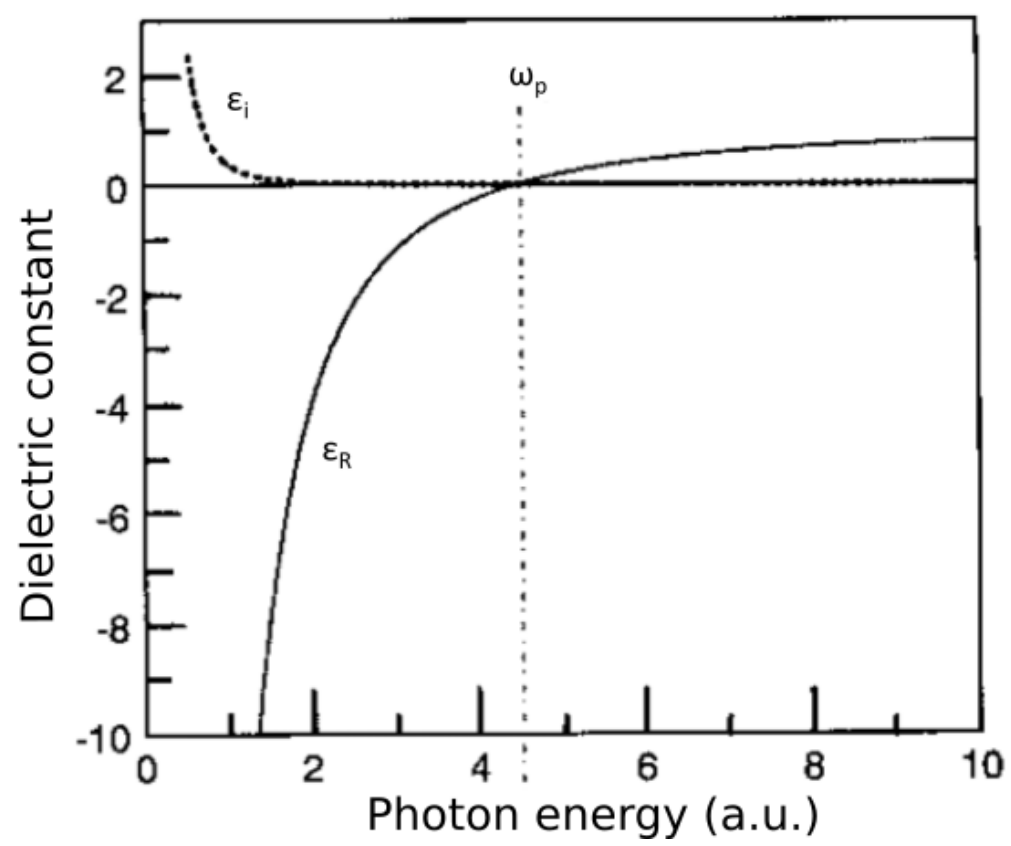

Figure 3.2. Dispersion of the real and imaginary parts of the dielectric constant [58].

dielectric constant becomes a positive value, meaning that metals act like a normal dielectric medium. The plasma frequency $\omega_{\tau}$ is defined by the point at which the real part changes sign.

Even though the Drude model is not expected to be appropriate for transition metals in the near IR. Oral et al. [59] introduced a Drude model parametrization of the dielectric function as a useful approximation for these metals. And the Drude model dielectric function is represented as:

$$
\tilde{\varepsilon_{r}}=\varepsilon_{\infty}-\frac{\omega_{p}^{2}}{\omega^{2}+i \omega \omega_{\tau}}
$$

where $\varepsilon_{\infty}$ is the high frequency contribution that is usually superior or equal to one. $\omega_{p}$ is the plasma frequency, $\omega_{\tau}$ is the damping frequency. Here $\omega, \omega_{p}, \omega_{\tau}$ have units 
of $\mathrm{cm}^{-1}$. The real part and imaginary part of the complex dielectric function are:

$$
\begin{gathered}
\varepsilon_{R}=\varepsilon_{\infty}-\frac{\omega_{p}^{2}}{\omega^{2}+\omega_{\tau}^{2}} \\
\varepsilon_{i}=\frac{\omega_{\tau} \omega_{p}^{2}}{\omega\left(\omega^{2}+\omega_{\tau}^{2}\right)}
\end{gathered}
$$

The plasma frequency is:

$$
\omega_{p}=\frac{1}{2 \pi c}\left(\frac{4 \pi N e^{2}}{m^{*}}\right)^{1 / 2}
$$

where $N$ is the free electron density, $e$ is the electronic charge, and $m^{*}$ is the effective mass of electrons.

The damping frequency $\omega_{\tau}$ is

$$
\omega_{\tau}=\frac{1}{2 \pi c \tau}
$$

where $\tau$ is the electron lifetime in seconds. In order to get the value of $\varepsilon_{R}$ and $\varepsilon_{i}$ with respect to frequency $(\omega)$, all the parameters in Eqn. 3.23 and 3.24 should be solved. Oral et al.'s fits showed that $\varepsilon_{\infty}$ is much less than $\varepsilon_{R}$ and $\varepsilon_{i}$, so $\varepsilon_{\infty}$ does not need to be taken as a third adjustable parameter in the fitting process. In the low frequency range, $\varepsilon_{\infty}$ is taken to be unity. The Drude model fit of the metals is shown in Table 3.1. So the expression of $\varepsilon_{R}$ vs. frequency and $\varepsilon_{i}$ vs. frequency can be obtained.

\subsection{Transmission simulation}

\subsubsection{Setup of transmission simulation}

The geometry of the 3D simulation unit is shown in Fig. 3.3. From the top to the bottom, the blocks are the perfect matched layer (PML), port 1, air, metamaterial unit, and port 2. The PML is a perfect absorber that will absorb unwanted reflections 
Table 3.1. Results of a drude model fit to the dielectric function of four Metals [59].

\begin{tabular}{lll}
\hline Metal & $10^{-2} \omega_{\tau}\left(\mathrm{cm}^{-1}\right)$ & $10^{-4} \omega_{\rho}\left(\mathrm{cm}^{-1}\right)$ \\
\hline $\mathrm{Cu}$ & 0.732 & 5.96 \\
$\mathrm{Al}$ & 6.60 & 11.9 \\
$\mathrm{Au}$ & 2.15 & 7.28 \\
$\mathrm{Ni}$ & 3.52 & 3.94 \\
\hline
\end{tabular}

in the system. Each metamaterial unit consists of three parts: a silicon substrate, a SU-8 pillar, and a layer of copper. Fig. 3.3(a), shows an isometric projection displaying the general finite element mesh. In Fig. 3.3(b), a $y-z$ planar cross-sectional plane near the center of the mesh displays each of the distinct regions of layered functional metamaterial structure required to simulate optoelectronic response to terahertz irradiation. A $1 \mathrm{~W}$ plane wave propagates from port 1 to port 2 . In Fig. 3.3(b), $d$ is the pillar height, $A p$ is the bottom side length, $P$ is the spacing between pillars, $d_{2}$ is the thickness of overplated copper, and $d_{3}$ is the distance of air layer.

The initial parameters of the geometry and the range of the radiation are shown in Table 3.2. The radiation frequency range is from 0.4 to $2 \mathrm{THz}$. Since there is a requirement of MMs that the feature size must be smaller or relative to the external wave we are interested in. Based on our target radiation range whose peak is at $1 \mathrm{THz}$, i.e., $300 \mu \mathrm{m}$ wavelength, all the feature size must be smaller than $300 \mu \mathrm{m}$. The customized mesh size was set relative to the minimum and maximun wavelength of the rerahertz radiation. Relative permittivity, relative permeability, and electrical conductivity are the required material properties for the simulation. For air and silicon, the material properties can be found in the COMSOL library. Since the relative permittivity of SU-8 and copper is frequency-dependent, their relative permittivities can be extrapolated from the data based on the Drude model $[59,60]$, as shown in Table 3.3. 
(a)

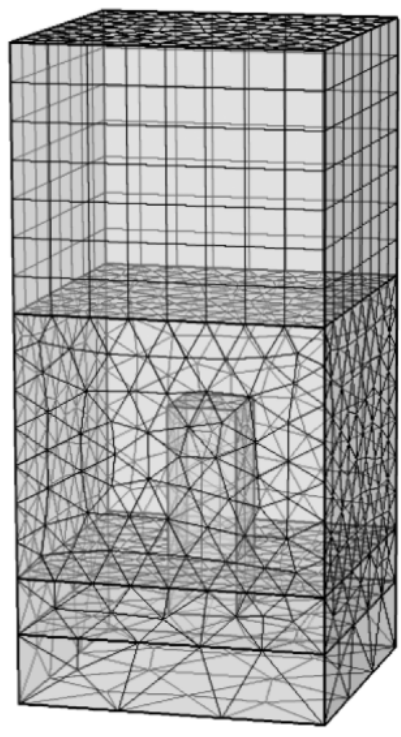

(b)

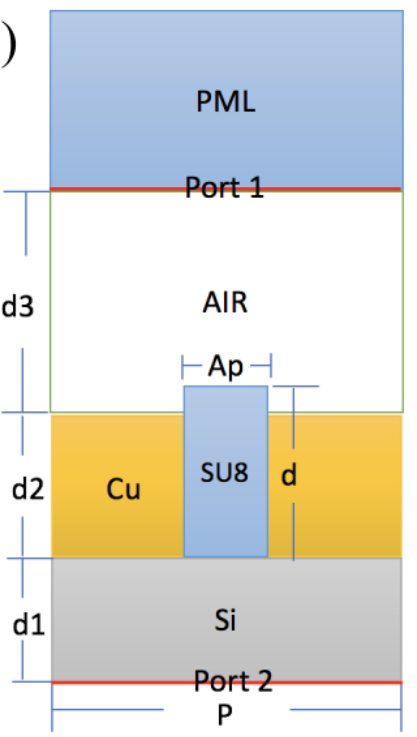

Figure 3.3. Geometry of a MM unit for COMSOL simulation.

Table 3.2. Parameters of COMSOL transmission simulation.

\begin{tabular}{llll}
\hline Name & Description & Expression & Value \\
\hline$A p$ & Bottom side length & $50 \times 10^{-6} \mathrm{~m}$ & $5 \times 10^{-5} \mathrm{~m}$ \\
$P$ & Pillar spacing & $220 \times 10^{-6} \mathrm{~m}$ & $2.2 \times 10^{-4} \mathrm{~m}$ \\
$d$ & Pillar hight & $150 \times 10^{-6} \mathrm{~m}$ & $1.5 \times 10^{-4} \mathrm{~m}$ \\
$d_{2}$ & Copper thickness & $40 \times 10^{-6} \mathrm{~m}$ & $4 \times 10^{-5} \mathrm{~m}$ \\
$d_{3}$ & Air thickness & c_const $^{-6} f_{\min } / 4$ & $1.8737 \times 10^{-4} \mathrm{~m}$ \\
$f_{\min }$ & Minimum frequency & $0.4 \times 10^{12} \mathrm{~Hz}$ & $4 \times 10^{11} \mathrm{~Hz}$ \\
$f_{\max }$ & Maximum frequency & $2 \times 10^{12} \mathrm{~Hz}$ & $2 \times 10^{12} \mathrm{~Hz}$ \\
$f p_{\mathrm{Cu}}$ & Drude model parameter 1 & $1.79 \times 10^{15} \mathrm{~Hz}$ & $1.79 \times 10^{15} \mathrm{~Hz}$ \\
$f t_{\mathrm{Cu}}$ & Drude model parameter 2 & $2.19 \times 10^{12} \mathrm{~Hz}$ & $2.19 \times 10^{12} \mathrm{~Hz}$ \\
$\lambda_{\min }$ & Minimum wavelength & $c_{-}$const $/ f_{\max }$ & $1.499 \times 10^{-4} \mathrm{~m}$ \\
$\lambda_{\max }$ & Maximum wavelength & $c_{-}$const $/ f_{\min }$ & $7.4948 \times 10^{-4} \mathrm{~m}$ \\
$d f$ & Step size of frequency & $0.1 \times 10^{12} \mathrm{~Hz}$ & $1 \times 10^{11} \mathrm{~Hz}$ \\
$S i_{\text {thick }}$ & Silicon thickness & $40 \times 10^{-6} \mathrm{~m}$ & $4 \times 10^{-5} \mathrm{~m}$ \\
\hline
\end{tabular}


Table 3.3. Properties of the materials used in the simulation.

\begin{tabular}{lcl}
\hline Property & Relative permeability, $\mu_{r}$ & Relative permittivity, $\varepsilon_{r}$ \\
\hline Air & 1 & 1 \\
SU-8 & 1 & $\varepsilon_{R(\mathrm{SU}-8)}(f)-i \varepsilon_{i(\mathrm{SU}-8)}(f)$ \\
Copper & 1 & $\varepsilon_{R(\mathrm{Cu})}(f)-i \varepsilon_{i(\mathrm{Cu})}(f)$ \\
Silicon & 1 & $3.418^{2}$ \\
\hline
\end{tabular}

\subsubsection{Results of transmission simulation}

Figures 3.4 and 3.5 are the experimental and simulation results of terahertz transmission test on metamaterials with $150 \mu \mathrm{m}$ thick pillars and different thickness of copper. In general, the two results show the same trend, that the transmission decreases with an increase in the copper thickness, and there appears to be a surface plasmon resonance around $1.2 \mathrm{THz}$. For the metamaterials with $150 \mu \mathrm{m}$ thick pillars and $40 \mu \mathrm{m}$ thick copper shown in Fig. 3.4, surface plasmon takes place at $1.17 \mathrm{THz}$, and $96 \%$ terahertz radiation is transmitted through the metamaterial. As the copper thickness increases to $75 \mu \mathrm{m}$ and $150 \mu \mathrm{m}$, the transmitted radiation peak drops to $90 \%$ and $45 \%$, respectively. When the copper thickness is $200 \mu \mathrm{m}$, terahertz radiation no longer transmits through the metamaterial. The disappearance of transmission is also observed in the simulation result in Fig. 3.5. However, there is a significant difference between the experimental and simulation results in that the transmission amplitude in the simulation is lower than that measured in the experiment. This is because, in simulation, defect-free metal lattices and the perfect attachment between metal/dielectric layers give rise to more reflection and absorption, resulting in less transmission. Moreover, the reflected portion is absorbed by the PML, preventing it from reflecting back. As a result, the amount of the radiation striking the metal/dielectric in the simulation is less than that of the experimental result, which give rise to a generally weaker transmission spectrum from the simulation. 


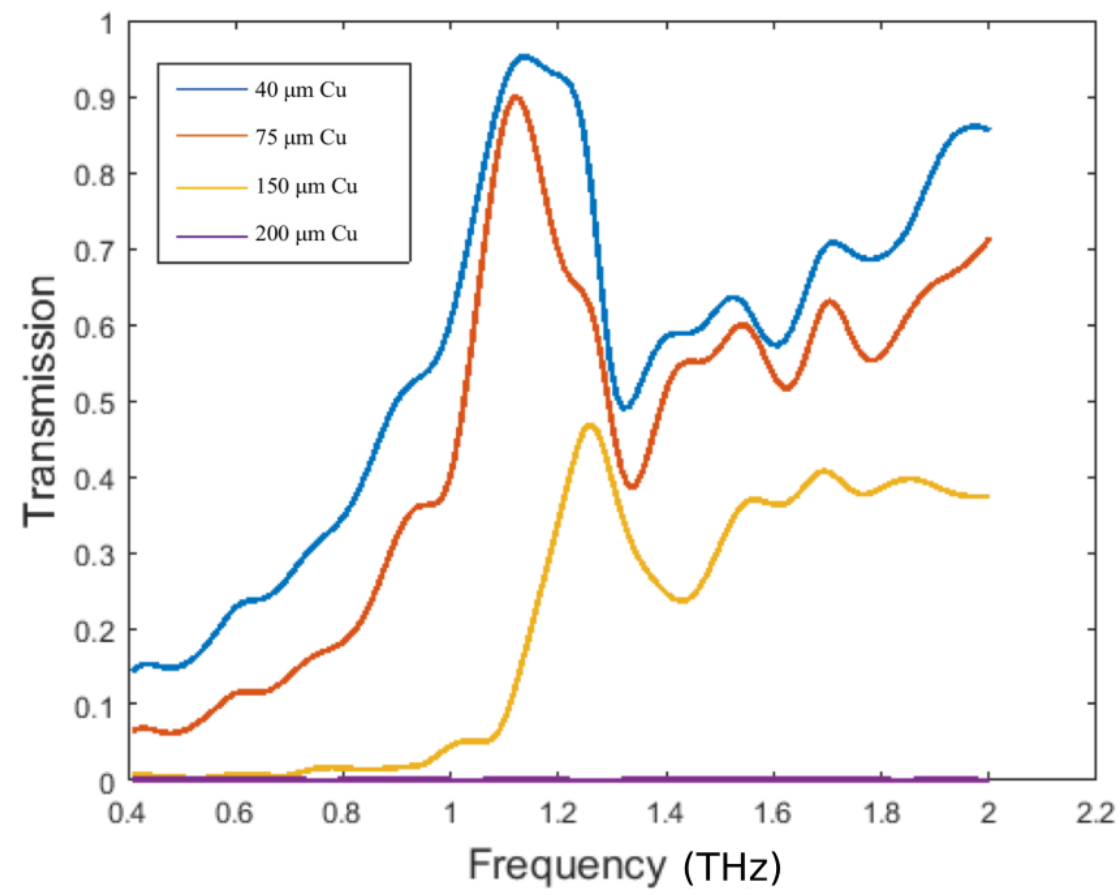

Figure 3.4. Experimental results of transmission of terahertz radiation through MMs from 0.4 to $2 \mathrm{THz}$ with different thicknesses of copper.

\subsection{Reflection simulation}

\subsubsection{Setup of reflection simulation}

In Fig. 3.6, the geometry setup of reflection is the same as that of the transmission simulation except the metamaterial unit is upside down so as to better display the localized surface plasmon at the metal/dielectric interface. There is still a $1 \mathrm{~W}$ plane wave propagating from port 1 to port 2 .

\subsubsection{Results of reflection simulation}

In order to optimize the geometry of the metamaterial, all the parameters in the system are investigated including the pillar height $(d)$, bottom side length $(A p)$, spacing between pillars $(P)$, the thickness of overplated copper $\left(d_{2}\right)$, the distance of air layer $\left(d_{3}\right)$ and the metal type. As shown in Figures 3.7, 3.8, and 3.9, even as we vary the metal type (copper, aluminum, gold and nickel), the thickness of air layer 


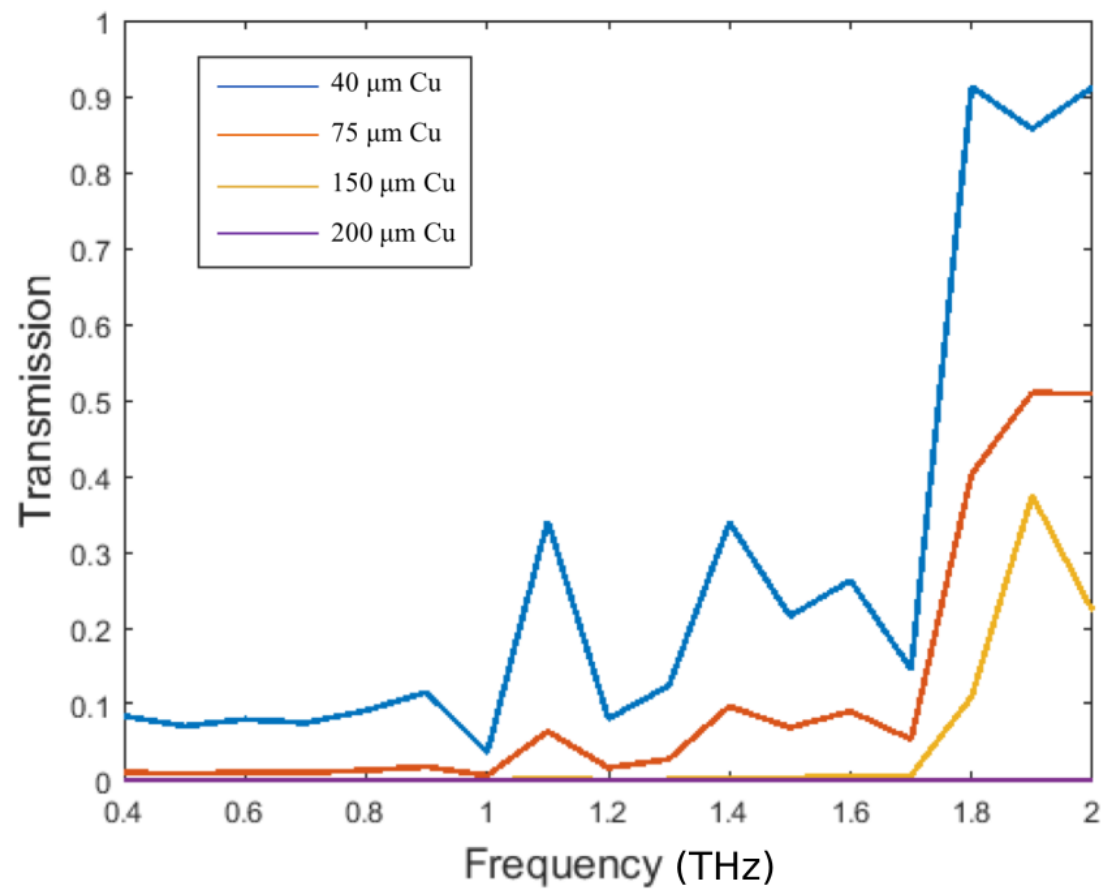

Figure 3.5. Simulation results of the transmission of terahertz radiation through MMs from 0.4 to $2 \mathrm{THz}$ with different thicknesses of copper.

(from $190 \mu \mathrm{m}$ to $750 \mu \mathrm{m}$ ) and the thickness of overplated copper (from $0.1 \mu \mathrm{m}$ to $40 \mu \mathrm{m})$, there is no change in the terahertz transmission spectrum. All of the spectra show three minimum points: $70 \%$ of the radiation is reflected at $1.2 \mathrm{THz}$ and $1.4 \mathrm{THz}$ reflectively, and $67 \%$ of the radiation is reflected at $1.8 \mathrm{THz}$. The independence of reflection to these parameters means that metal only acts as a medium to transfer the propagating surface plasmon, so the difference of the electrical conductivity between different metals will not affect the reflection property of the metamaterial.

However, the reflection property of the metamaterial changes significantly if the SU-8-related parameters $(d, A p$, and $P$ ) are changed, as shown in Fig. 3.10, 3.11, and 3.12. As shown in Fig. 3.10, with an increase in the pillar height $(d)$ from $50 \mu \mathrm{m}$ to $125 \mathrm{\mu m}$, the minimum reflection of the metamaterial shifts from the high frequency to low frequency, indicating the surface plasmon resonance is also moving from high 

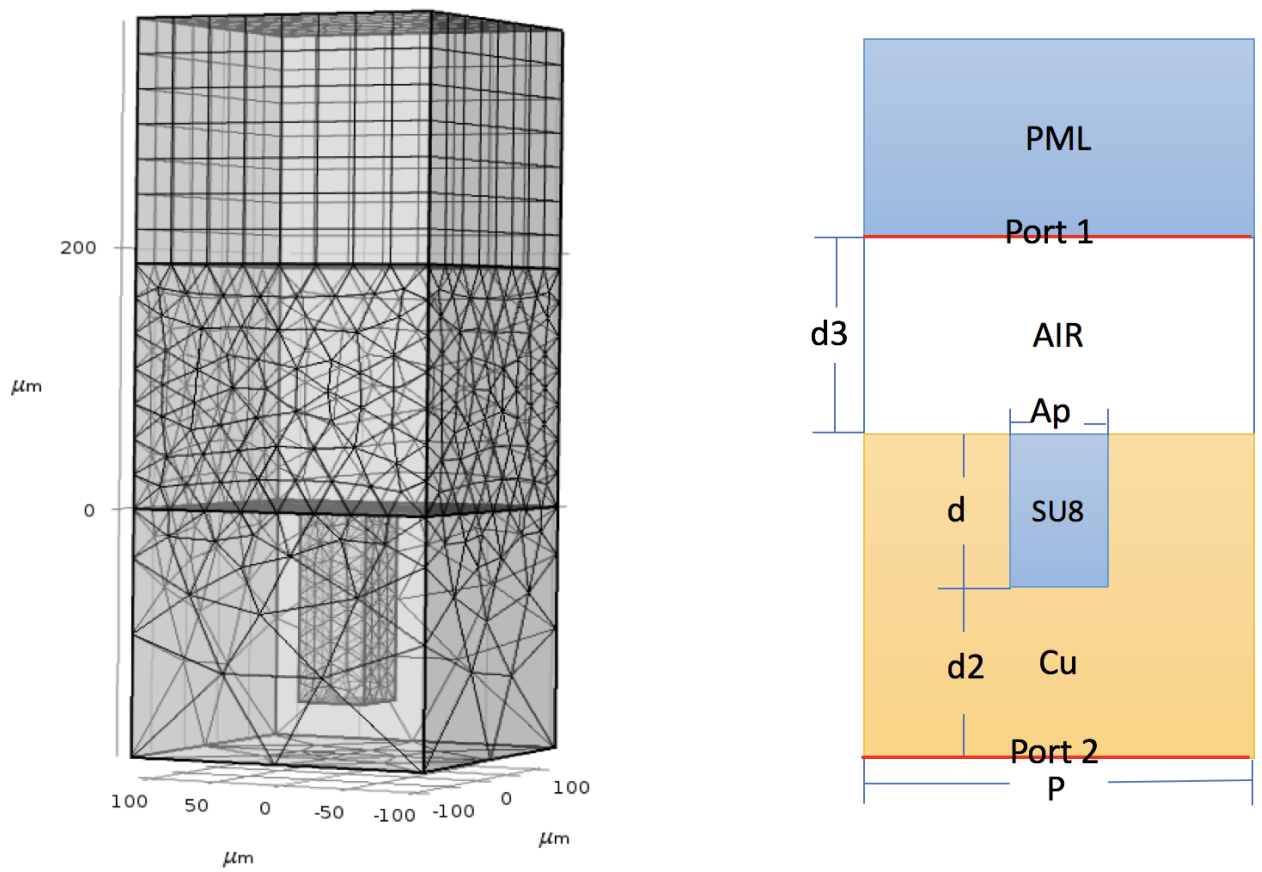

Figure 3.6. Geometry of a MM unit for reflection simulations.

frequency to low frequency; and the minimum reflection is $39 \%$ that takes place at 1.2 THz when the pillar height is $100 \mu \mathrm{m}$. As shown in Fig. 3.11, the increase of the bottom side length $(A p)$ from $40 \mu \mathrm{m}$ to $70 \mu \mathrm{m}$ gives rise to a decrease of terahertz pulse reflection from MMs, and there is a minimum reflection, 63\%, taking place at $1.3 \mathrm{THz}$. In addition, the increase of the spacing $(P)$ between pillars from $120 \mu \mathrm{m}$ to $200 \mu \mathrm{m}$ would increase the reflection on metamaterials, as shown in Fig. 3.12. When we combine the results from Fig. 3.11 and Fig. 3.12, we found that with the increase of surface area ratio of $\mathrm{SU}-8 / \mathrm{Cu}$, the terahertz reflection to MMs decreases.

After considering each of the parameters from all perspectives, including the absorption efficiency of metamaterial, the ease of fabrication and characterization, the compatibility between the metamaterial and the diode, etc., the geometry of our 3D metamaterial is optimized as shown in Fig. 3.13. The pillar height is $150 \mu \mathrm{m}$, the aperture dimension is $80 \mu \mathrm{m}$, the spacing between pillars is $220 \mu \mathrm{m}$, and the thickness 


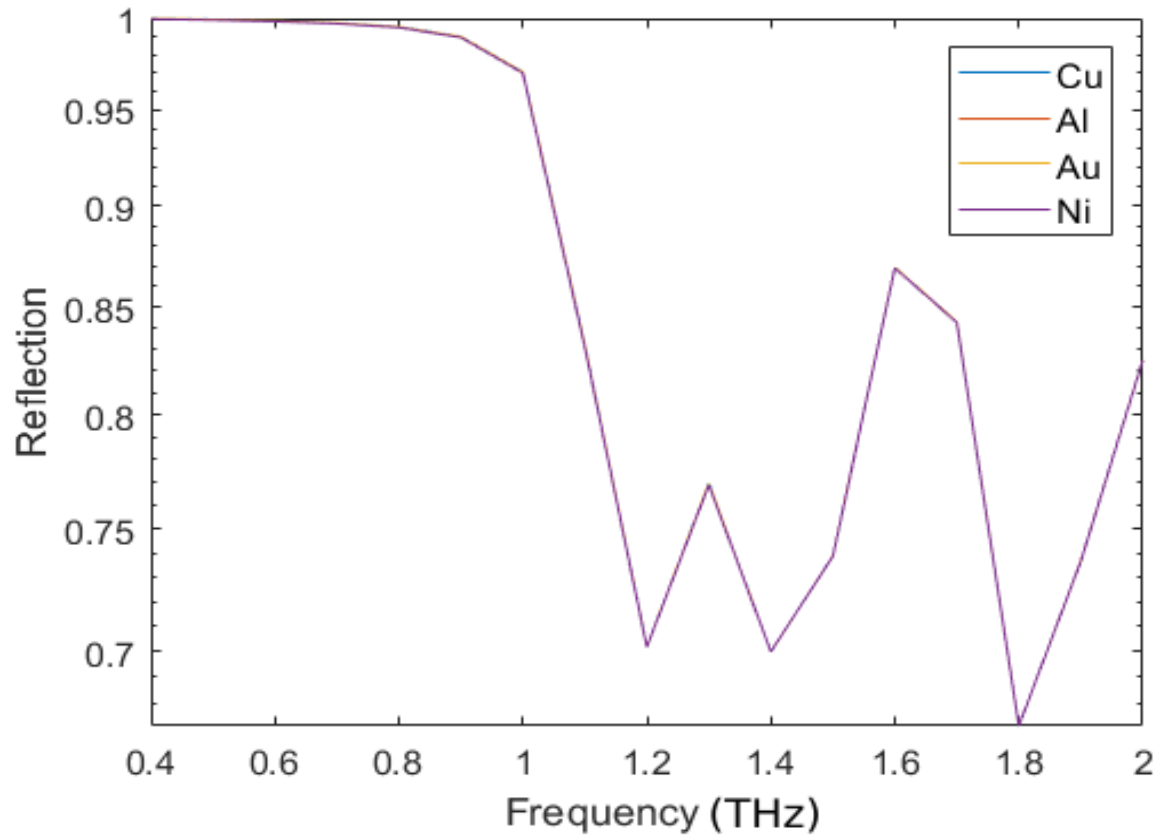

Figure 3.7. Reflection of MMs from 0.4 to $2 \mathrm{THz}$ with different metals. Please note that all the curves overlap together.

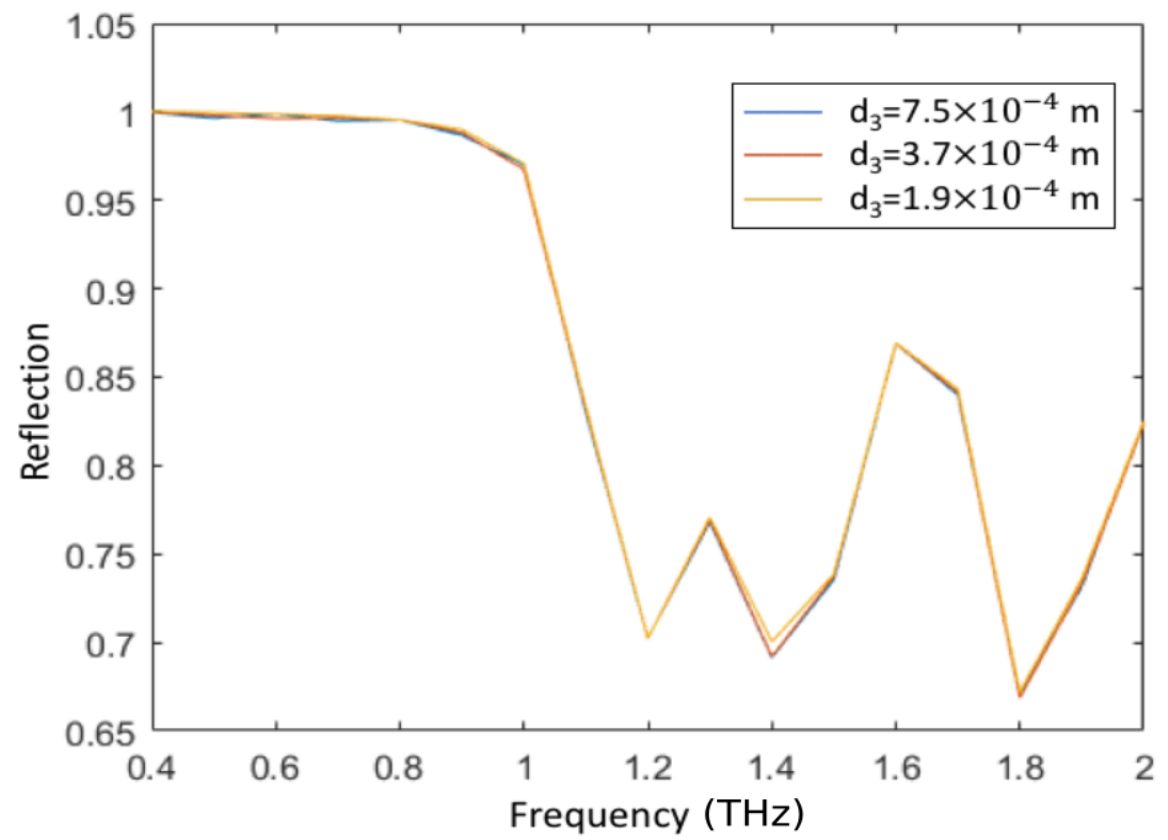

Figure 3.8. Reflection of MMs from 0.4 to $2 \mathrm{THz}$ with different air distance $d_{3}$. Please note that all the curves overlap together. 


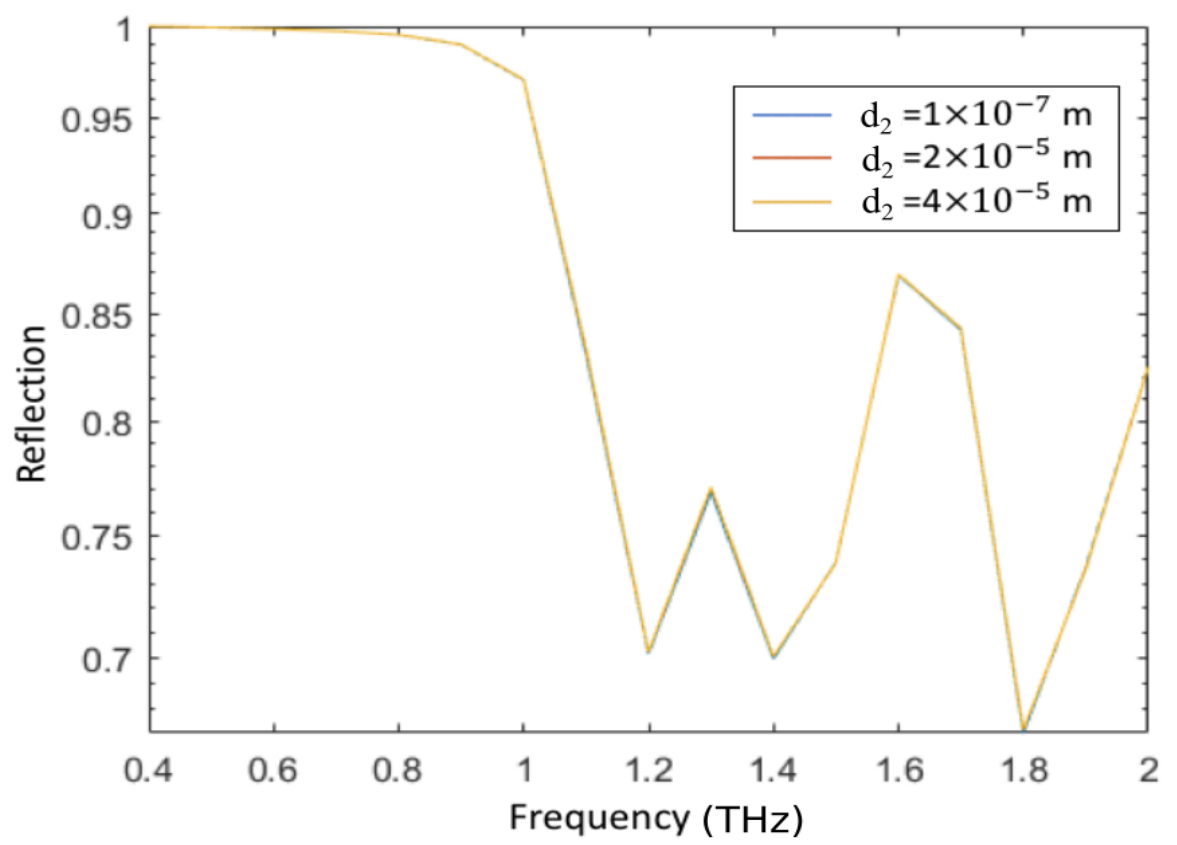

Figure 3.9. Reflection of MMs from 0.4 to $2 \mathrm{THz}$ with different overplated copper thickness $d_{4}$. Please note that all the curves overlap together.

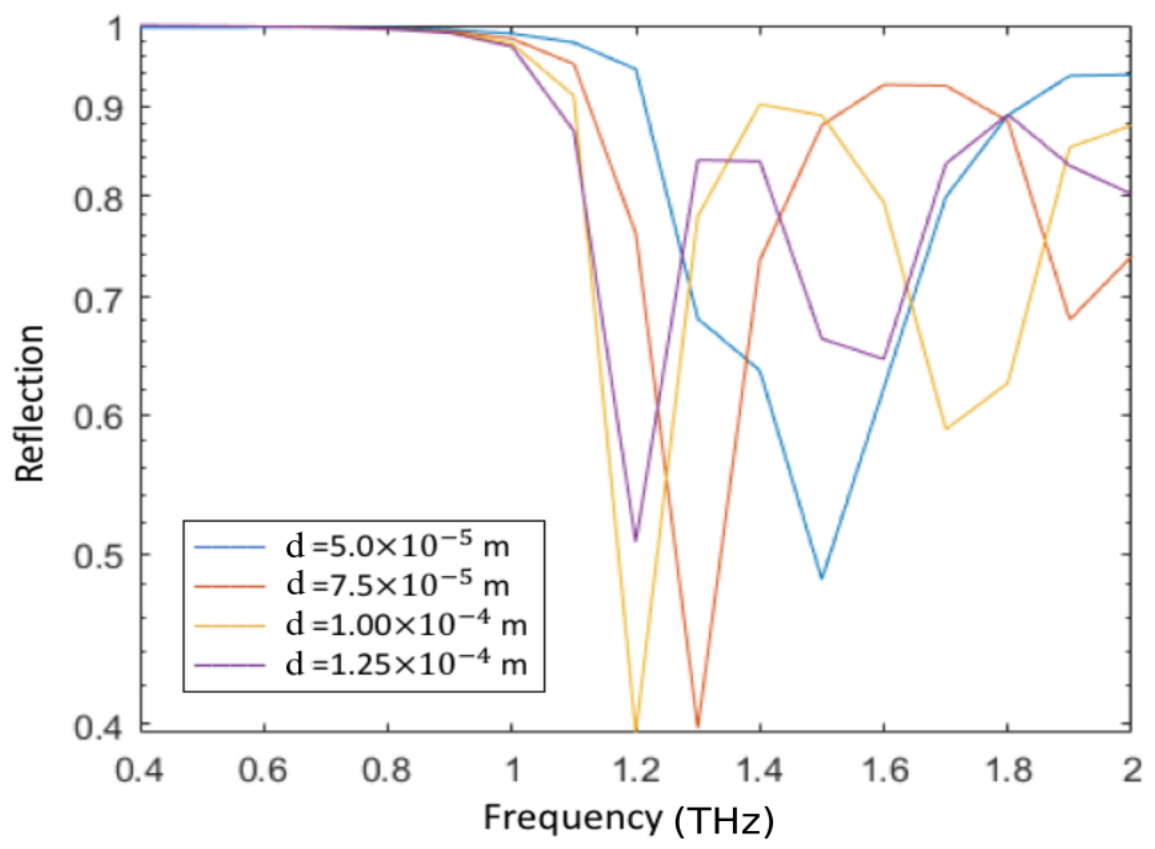

Figure 3.10. Reflection of MMs from 0.4 to $2 \mathrm{THz}$ with different SU-8 pillar height $d$. 


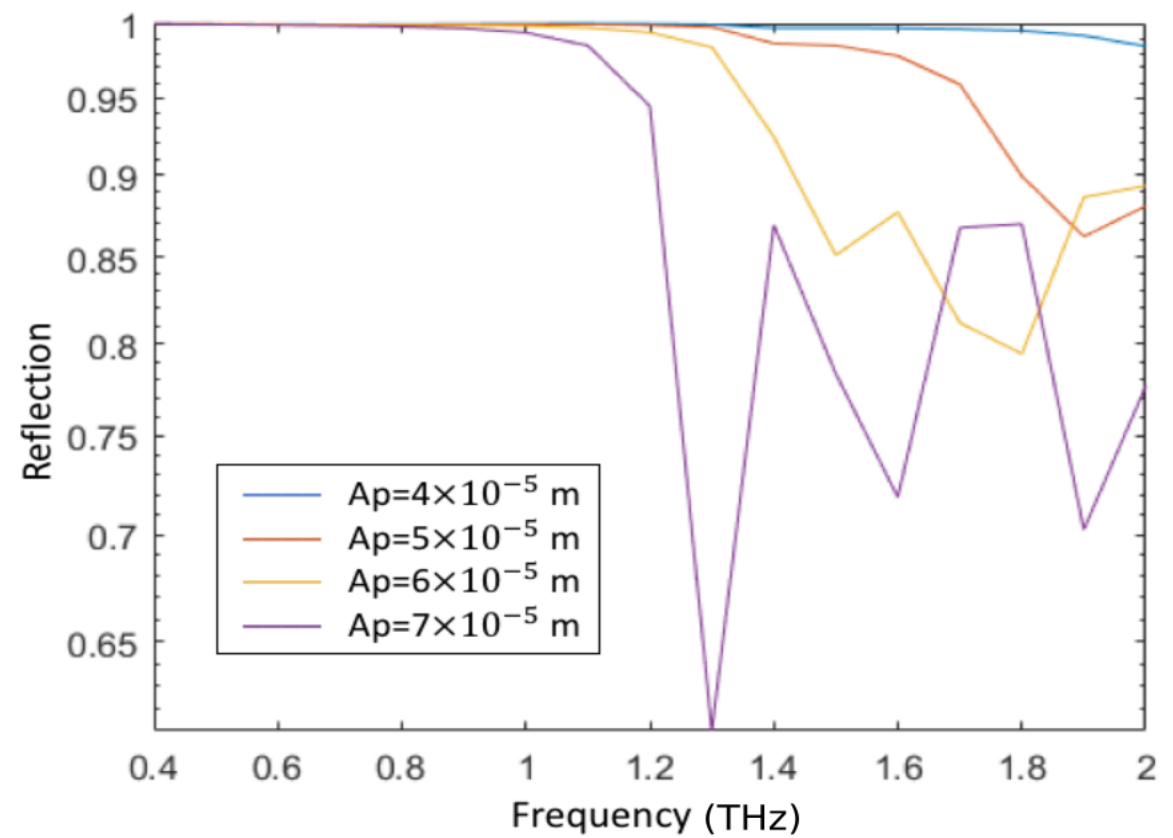

Figure 3.11. Reflection of MMs from 0.4 to $2 \mathrm{THz}$ with different SU-8 width $A p$.

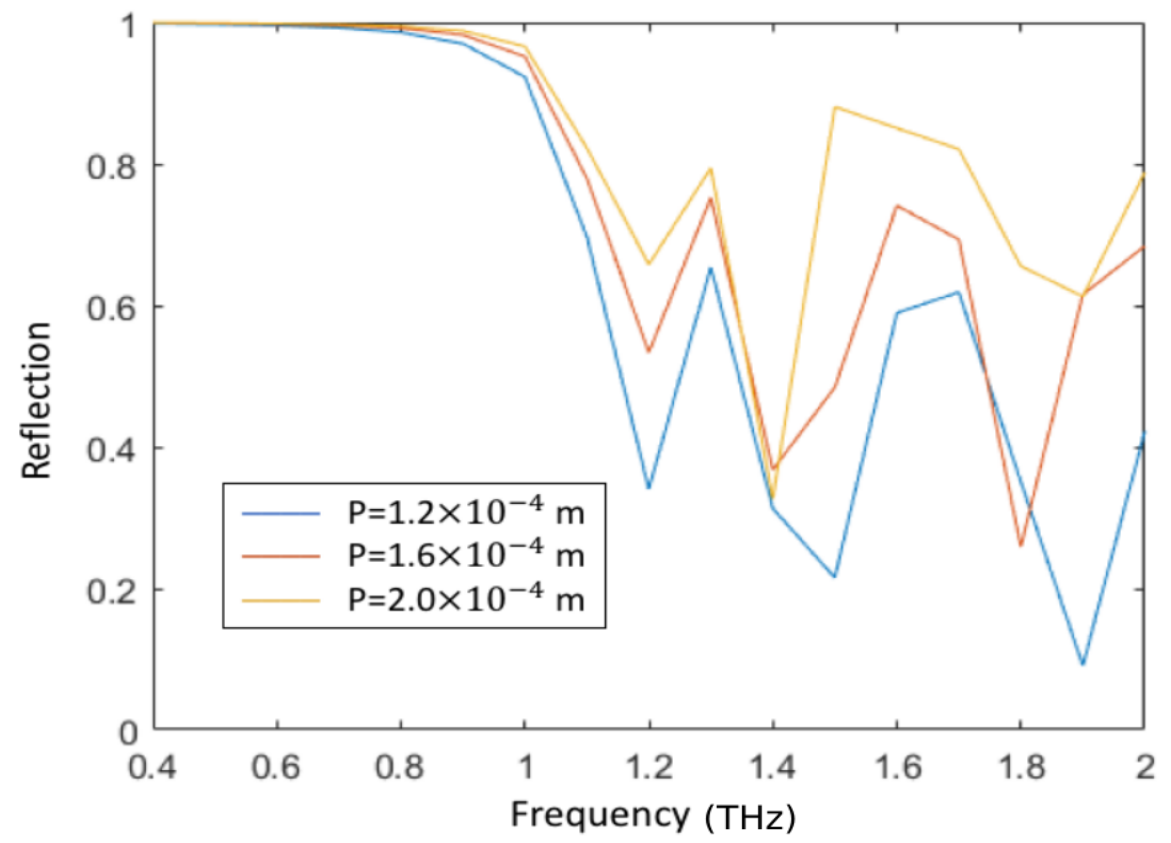

Figure 3.12. Reflection of MMs from 0.4 to $2 \mathrm{THz}$ with different $\mathrm{MM}$ unit spacing $P$. 


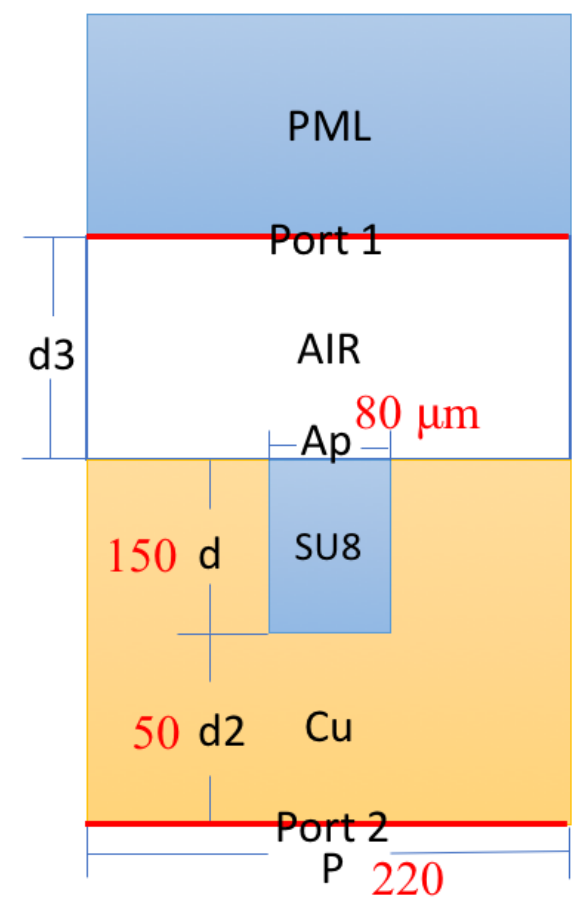

Figure 3.13. Final optimized geometry of 3D metamaterial unit.

of overplated copper is $50 \mu \mathrm{m}$. In this geometry, the transmission portion is zero, so it is easy to characterize the reflection and absorption property of MMs. Copper is the metallic material of choice in the metamaterial due to its high thermal and electrical conductivity, excellent mechanical properties, relative cost, and availability.

Figure 3.14 shows the reflection spectrum of the MMs with the optimized geometry. It is clear that there are three major surface plasmon resonance peaks observed at frequencied of $1.0 \mathrm{THz}(70 \%), 1.2 \mathrm{THz}(70 \%)$ and $1.8 \mathrm{THz}(68 \%)$. The electric field distribution of the optimized metamaterials at different frequencies is shown in Fig. 3.15. The red color represents the highest electric field region. Figure 3.15(b) shows that the highest electric field is located inside the dielectric pillar and also at the metal/dielectric/air interface which is also the location to position the nano-antenna in the future, however, the radiation of higher or lower frequency can not be focused to the MMs. So we conclude that the optimized geometry can localize the surface 


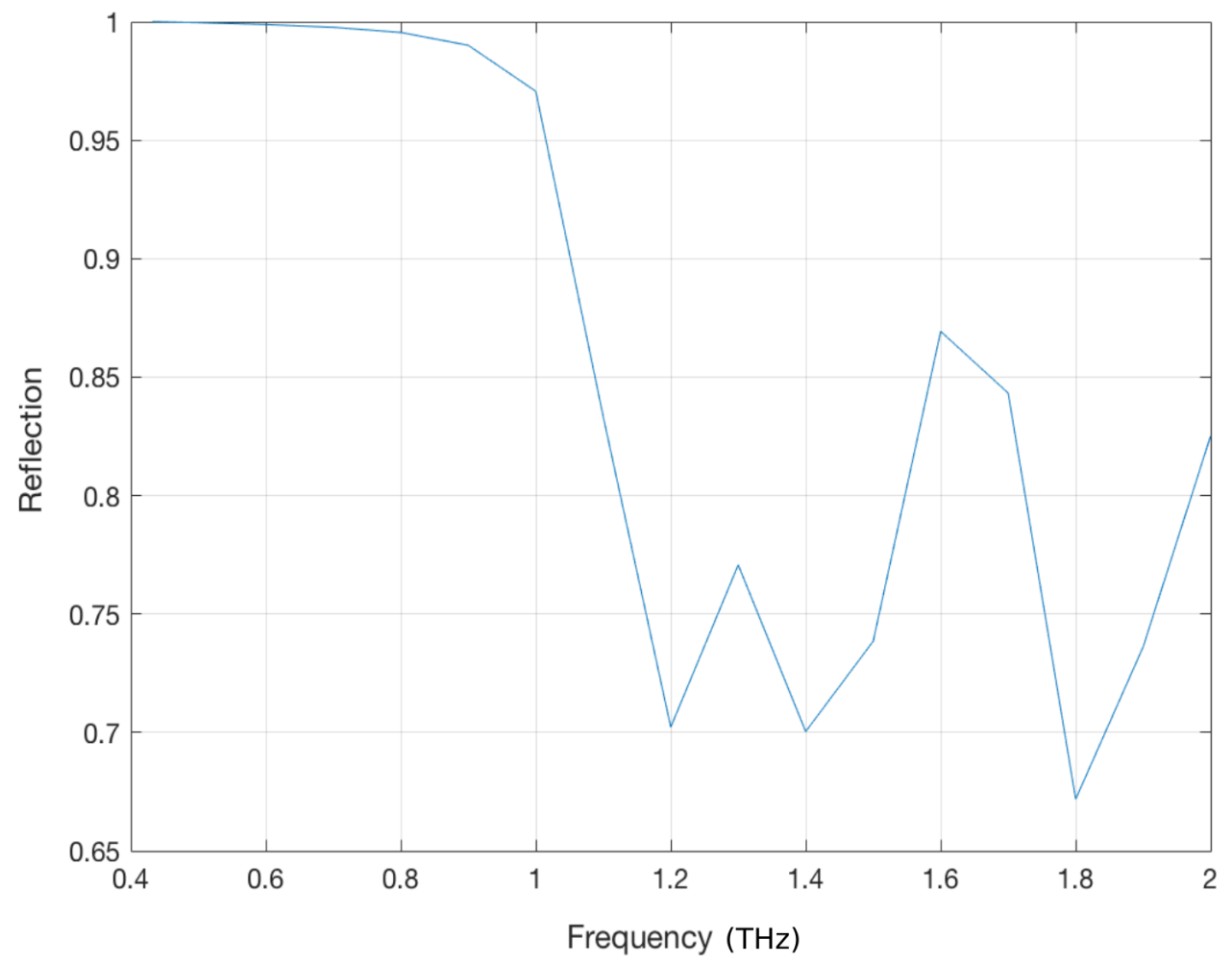

Figure 3.14. Reflection of the optimized metamaterial from 0.4 to $2 \mathrm{THz}$.

plasmon with the peak frequency of $1.2 \mathrm{THz}$ and reflection intensity of $70 \%$. This means that the highest terahertz absorption is $30 \%$ which takes place at $1.2 \mathrm{THz}$.

\subsection{Conclusions}

A series of FEM COMSOL simulations were carried out to investigate the transmission and reflection properties of metamaterial. In terms of the transmission of terahertz radiation, the simulation and experimental results show the same trend that the transmission decreases with an increase in the copper thickness. Moreover, no terahertz radiation transmits through the metamaterial with $150 \mu \mathrm{m}$ thick pillar and $200 \mu \mathrm{m}$ thick copper.

The reflection simulation shows that the reflection to metamaterial is dependent on SU-8 related parameters, including pillar height, bottom side length and spacing 
(a)

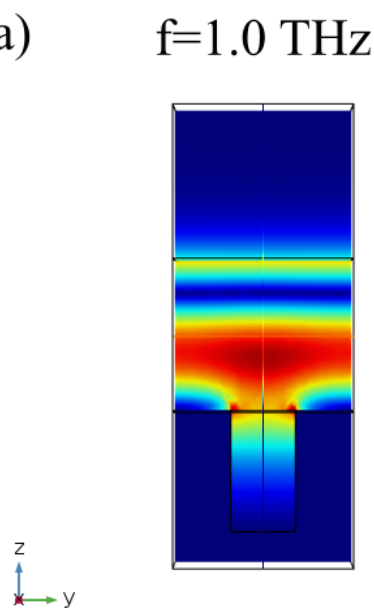

(c)

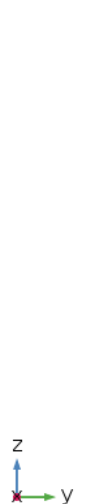

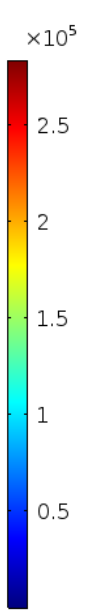
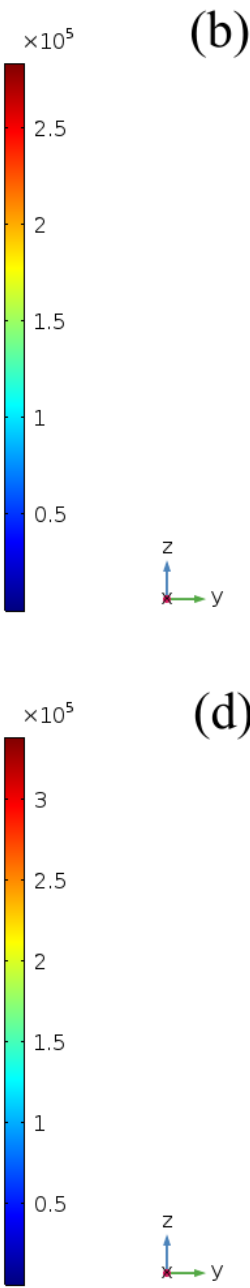

(b) $\mathrm{f}=1.2 \mathrm{THz}$
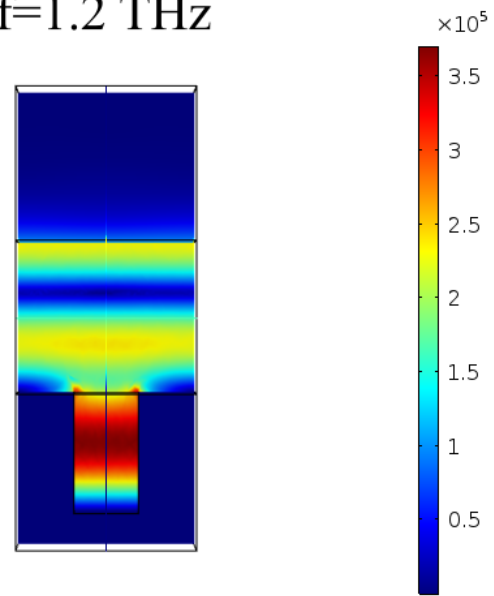

(d) $\mathrm{f}=1.8 \mathrm{THz}$

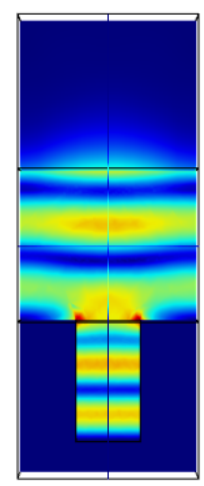

Figure 3.15. The electric field distribution of the optimized metamaterials at different radiation frequencies. From panels (a) to (d), the frequency is 1.0, 1.2, 1.4, and $1.8 \mathrm{THz}$, respectively. 
between pillars, while the reflection is independent on the thickness of overplated copper, the distance of air layer and the metal type. We also found that with the increase of surface area ratio of $\mathrm{SU}-8 / \mathrm{Cu}$, the terahertz reflection to MMs decreases. Finally, the optimized metamaterial geometry is achieved and it can localize the surface plasmon with the peak frequency of $1.2 \mathrm{THz}$ to the nano-antenna and the highest terahertz absorption is $30 \%$. 


\section{CHAPTER 4}

\section{SUMMARY AND CONCLUDING REMARKS}

\subsection{Conclusions}

My work has achieved a series of concluding remarks based on metamaterials in three aspects. First, three-dimensional metamaterials for terahertz energy harvesting were successfully manufactured. In parallel, FEM COMSOL simulation was carried out as a demonstration to visualize the mechanism involved in the experiments and also as a prediction tool for metamaterials design. Finally, THz-TDS characterization was used to characterize the transmission performance of metamaterials.

\subsubsection{D THz metamaterial fabrication}

We have successfully created this comprehensive protocol for the fabrication of three dimensional metamaterials. A series of experiments was conducted involving the electrodeposition of copper for the fabrication of metamaterials under different conditions. In our work, Cl-MPSA-PEG-PVP additives can interplay with each other well and they are effective in both bottom-up superfill and leveling for electrodeposition of copper for metamaterials. In addition, levelers would not impact the PEGCl-MPSA bottom-up filling. Compared with PVP, another leveler, Janus Green B exhibits a better leveling effect. NMR and FTIR data show that even without current flow, the accelerator MPSA converts to SPS in acid copper bath immediately; The process is not reversible under our experimental conditions. When there are $\mathrm{Cl}^{-}$, MPSA, PEG, and PVP additives in the acid copper solution, accelerators interact 
with these additives and form complexes, resulting in the breakdown of accelerators and a decrease in the concentration; with current flow, this degradation process can be enhanced.

\subsubsection{FEM simulation of metamaterial}

\subsubsection{Electrochemical simulation}

2D FEM of electrodeposition of copper demonstrate the growth of copper film and the change of concentration and overpotential during electrodeposition. During electrodeposition, the concentration of the bulk electrolyte is the same as the initial concentration. The cathode has the lowest concentration of copper while the anode has the highest because the consumable copper anode releases copper ions to the solution.

Superfilling simulation shows a real-time profile of the deposition of copper into a trench, which provides a better understanding of the bottom-up process and reveals the cause of overplating and the movement of accelerators in the trench. The accelerator species may initially accumulate on the bottom of the trench and be transported vertically until overplating, whereby they are transported laterally. The simulation results agree with our experimental results.

\subsubsection{Electromagnetic simulation}

3D transmission and reflection simulations provide an optimization of MMs geometry design. Our study shows that pillar geometry has a significant impact on the performance of MMs while other parameters including the thickness of overplated copper, spacing of pillars and metal type have no effect. The optimized metamaterial can localize the surface plasmon with the peak frequency of $1.2 \mathrm{THz}$ to the nano-antenna and the highest terahertz absorption is $30 \%$. 


\subsubsection{Electromagnetic characterization}

In order to investigate the interaction of $1 \mathrm{THz}$ radiation with the metamaterial, a terahertz time-domain spectroscopy (THz-TDS) is used to measure the transmission of the metamaterial. THz-TDS scan shows homogenous absorption throughout the whole metamaterial surface. Moreover, there appears a sharp absorption peak at $1.2 \mathrm{THz}$ indicating a strong surface plasmon resonance at $1.2 \mathrm{THz}$. The geometries of the metamaterials, including the height and the spacing of SU-8 pillars, the thickness of copper, the flatness of the metamaterial surface, have an impact on the transmission. In combination with FEM simulation of transmission and reflection, an optimization of the metamaterial geometry is achieved. On the way to exploring the optimal geometry, we found that MMs features are able to influence THz transmission and the thickness of MMs plays the most important role. At $1 \mathrm{THz}$, a strong correlation appears between transmitted E-Field magnitude and copper thickness. Above $1 \mathrm{THz}, \mathrm{EM}$ wave transmission is more easily facilitated because the thickness of copper has little effect. Compared to $40 \mu \mathrm{m}$ pillars, $150 \mu \mathrm{m}$ pillars provide enhanced resonance to MMs at $1 \mathrm{THz}$.

\subsection{Future work}

In the future, we plan to measure the reflectivity of the metamaterial and characterize its capability as an absorber. After the characterization of MMs is finished, we will assemble the MIM diode, nano-antenna, and metamaterial together and characterize the performance of the energy harvesting device.

Even though we have successfully conducted a series of 2D COMSOL simulations of electrodeposition and investigated the mechanism of basic electrodeposition, the superfilling of features and adsorption of accelerators, we will also move onto 3D COMSOL simulation in the future. 
In order to have a better leveling effect for the top surface of the deposits, we have been designing and synthesizing other levelers based on the effect of the functional groups. There are still a lot of future work need to be finished.

First, we plan to find a new carboxylic acid as the diacid monomer for the polymerization reaction. Since the current diacid reagent, dipicolinic acid has a melting point $244{ }^{\circ} \mathrm{C}$ at which it also decomposed. For this polymerization reaction, the diacid needs to melt first but it is hard to achieve that without damaging its chemical structure. Also, it is hard to dissolve dipicolinic acid in most of the organic solvent. So it is better to find an alternative diacid with the desired properties including: low melting point, high stability, nitrogen-containing and $\pi$-bond containing.

Secondly, since polyesters degrade in water after a long time, polyanhydrides and polyamides are likely to be more stable. So those two types of levelers are also going to be synthesized. Scheme 4.1 and 4.2 are the synthetic routes for polyanhydrides and polyamides, respectively. 


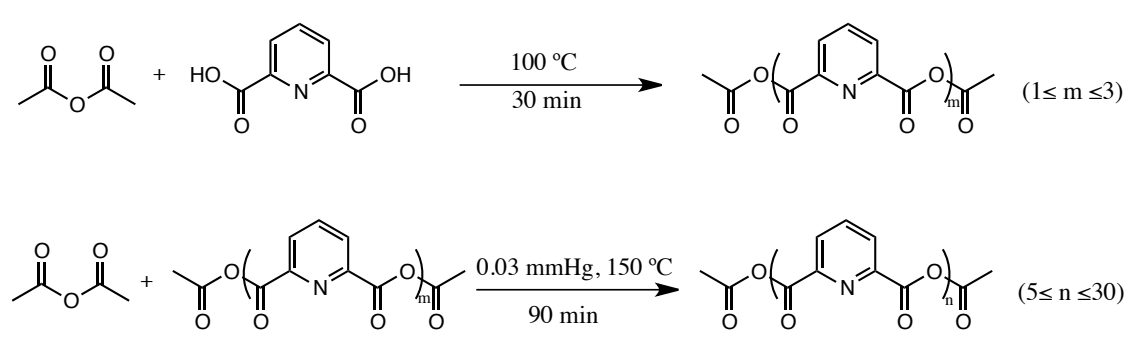

Scheme 4.1. Polymerization reaction of polyanhydride based leveler.
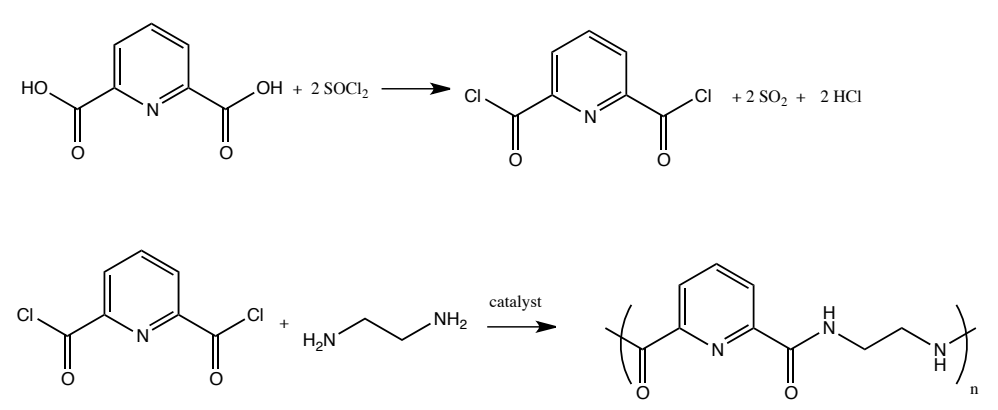

Scheme 4.2. The polymerization reaction of polyamide based leveler. The catalyst candidates are DCC, HBTU, TBTU and PyBOP. 


\section{APPENDIX A}

\section{FOURIER TRANSFORM FOR THZ-TDS}

The following is the code for THz-TDS data processing.

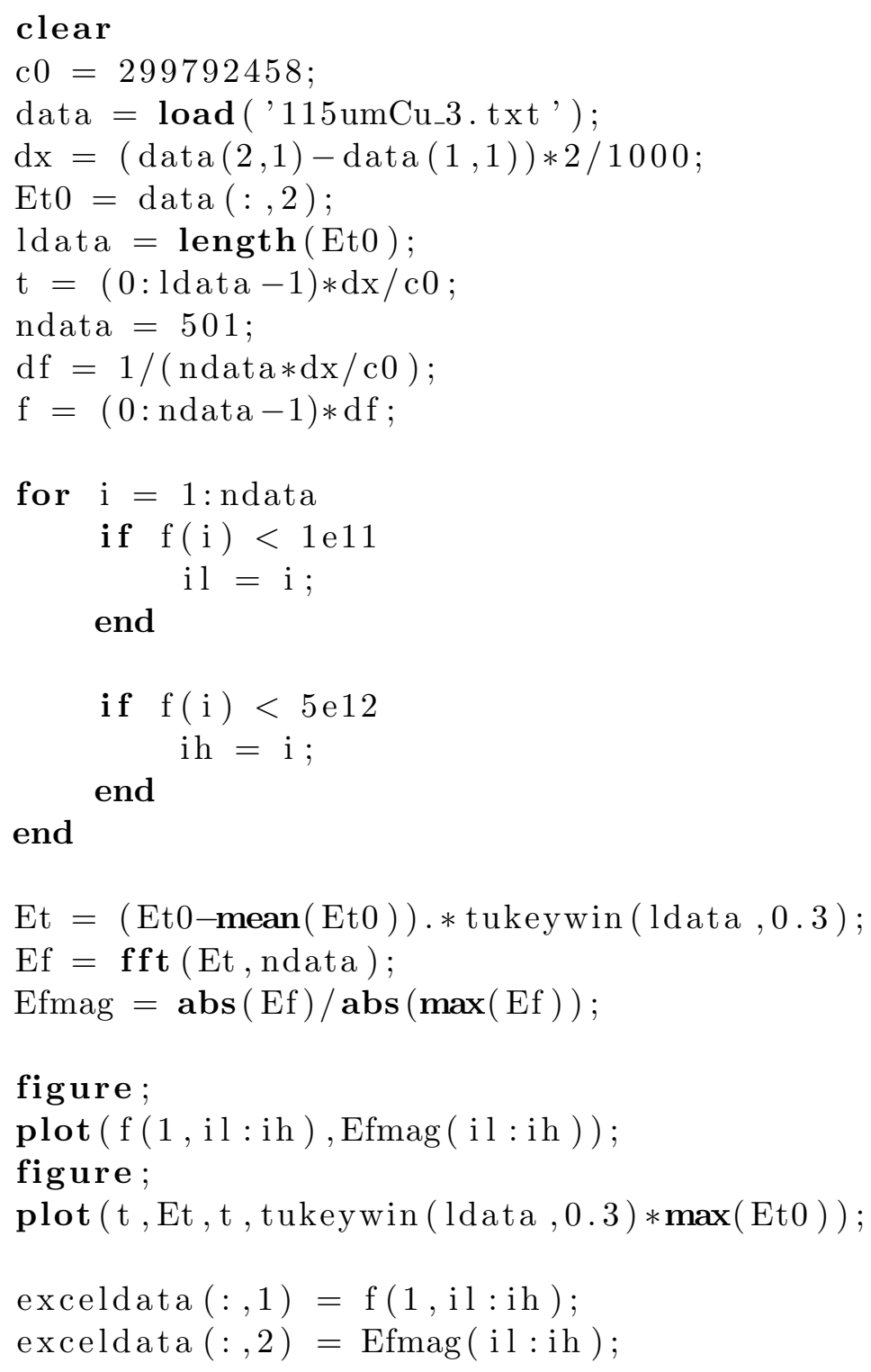




\section{BIBLIOGRAPHY}

[1] S. Zhang, W. Fan, N. Panoiu, K. Malloy, R. Osgood, and S. Brueck, "Experimental demonstration of near-infrared negative-index metamaterials," Physical Review Letters, vol. 95, no. 13, p. 137404, 2005.

[2] N. I. Zheludev, "The road ahead for metamaterials," Science, vol. 328, no. 5978, pp. 582-583, 2010.

[3] V. G. Veselago, "The electrodynamics of substances with simultaneously negative values of and $\mu, "$ Advances in Physical Sciences, vol. 10, no. 4, p. 509, 1968.

[4] D. R. Smith, W. J. Padilla, D. Vier, S. C. Nemat-Nasser, and S. Schultz, "Composite medium with simultaneously negative permeability and permittivity," Physics Review Letters, vol. 84, no. 18, p. 4184, 2000.

[5] M. Wiltshire, J. Pendry, I. Young, D. Larkman, D. Gilderdale, and J. Hajnal, "Microstructured magnetic materials for RF flux guides in magnetic resonance imaging," Science, vol. 291, no. 5505, pp. 849-851, 2001.

[6] D. R. Smith and N. Kroll, "Negative refractive index in left-handed materials," Physics Review Letters, vol. 85, no. 14, p. 2933, 2000.

[7] M. Gokkavas, K. Guven, I. Bulu, K. Aydin, R. Penciu, M. Kafesaki, C. Soukoulis, and E. Ozbay, "Experimental demonstration of a left-handed metamaterial operating at $100 \mathrm{GHz}, "$ Physical Review B, vol. 73, no. 19, p. 193103, 2006 .

[8] T.-J. Yen, W. Padilla, N. Fang, D. Vier, D. Smith, J. Pendry, D. Basov, and $\mathrm{X}$. Zhang, "Terahertz magnetic response from artificial materials," Science, vol. 303, no. 5663, pp. 1494-1496, 2004.

[9] S. Linden, C. Enkrich, M. Wegener, J. Zhou, T. Koschny, and C. M. Soukoulis, "Magnetic response of metamaterials at 100 terahertz," Science, vol. 306, no. 5700, pp. 1351-1353, 2004.

[10] G. Dolling, M. Wegener, C. M. Soukoulis, and S. Linden, "Negative-index metamaterial at $780 \mathrm{~nm}$ wavelength," Optics Letters, vol. 32, no. 1, pp. 53-55, 2007. 
[11] J. Hao, J. Wang, X. Liu, W. J. Padilla, L. Zhou, and M. Qiu, "High performance optical absorber based on a plasmonic metamaterial," Applied Physics Letters, vol. 96, no. 25, p. 251104, 2010.

[12] Y. Liu, Y. Chen, J. Li, T.-C. Hung, and J. Li, "Study of energy absorption on solar cell using metamaterials," Solar Energy, vol. 86, no. 5, pp. 1586-1599, 2012 .

[13] D. M. Mattox, Handbook of Physical Vapor Deposition (PVD) Processing. William Andrew, 2010.

[14] M. Paunovic, "Electroless deposition of copper," Modern Electroplating, vol. 1, pp. 433-446, 2010.

[15] H.-G. Creutz, R. W. Herr, et al., "Electrodeposition of copper," 1978. US Patent 4,110,176.

[16] A. J. Bard, L. R. Faulkner, J. Leddy, and C. G. Zoski, Electrochemical Methods: Fundamentals and Applications, vol. 2. Wiley New York, 1980.

[17] T. Moffat, D. Wheeler, W. Huber, and D. Josell, "Superconformal electrodeposition of copper," Electrochemical and Solid-State Letters, vol. 4, no. 4, pp. C26C29, 2001.

[18] M. Lefebvre, G. Allardyce, M. Seita, H. Tsuchida, M. Kusaka, and S. Hayashi, "Copper electroplating technology for microvia filling," Circuit World, vol. 29, no. 2, pp. 9-14, 2003.

[19] P. Moçotéguy, C. Gabrielli, H. Perrot, A. Zdunek, and D. N. Sanz, "Influence of the anode and the accelerator on copper bath aging in the damascene process," Journal of The Electrochemical Society, vol. 153, no. 12, pp. G1086-G1098, 2006 .

[20] P. C. Andricacos, C. Uzoh, J. O. Dukovic, J. Horkans, and H. Deligianni, "Damascene copper electroplating for chip interconnections," IBM Journal of Research and Development, vol. 42, no. 5, pp. 567-574, 1998.

[21] T. Moffat, J. Bonevich, W. Huber, A. Stanishevsky, D. Kelly, G. Stafford, and D. Josell, "Superconformal electrodeposition of copper in 500-90 nm features," Journal of The Electrochemical Society, vol. 147, no. 12, pp. 4524-4535, 2000.

[22] M. Tan, C. Guymon, D. R. Wheeler, and J. N. Harb, "The role of SPS, MPSA, and chloride in additive systems for copper electrodeposition," Journal of The Electrochemical Society, vol. 154, no. 2, pp. D78-D81, 2007.

[23] T. Moffat, B. Baker, D. Wheeler, and D. Josell, "Accelerator aging effects during copper electrodeposition," Electrochemical and Solid-State Letters, vol. 6, no. 4, pp. C59-C62, 2003. 
[24] A.-Y. Wang, B. Chen, L. Fang, J.-J. Yu, and L.-M. Wang, "Influence of branched quaternary ammonium surfactant molecules as levelers for copper electroplating from acidic sulfate bath," Electrochimica Acta, vol. 108, pp. 698706, 2013.

[25] W.-P. Dow, C.-C. Li, Y.-C. Su, S.-P. Shen, C.-C. Huang, C. Lee, B. Hsu, and S. Hsu, "Microvia filling by copper electroplating using diazine black as a leveler," Electrochimica Acta, vol. 54, no. 24, pp. 5894-5901, 2009.

[26] M. J. Willey, J. Reid, and A. C. West, "Adsorption kinetics of polyvinylpyrrolidone during copper electrodeposition," Electrochemical and Solid-State Letters, vol. 10, no. 4, pp. D38-D41, 2007.

[27] J. D. Reid and J. Zhou, "Impact of leveler molecular weight and concentration on damascene copper electroplating," ECS Transactions, vol. 2, no. 6, pp. 7792, 2007 .

[28] L.-B. Zhao, R. Huang, M.-X. Bai, D.-Y. Wu, and Z.-Q. Tian, "Effect of aromatic amine- metal interaction on surface vibrational raman spectroscopy of adsorbed molecules investigated by density functional theory," The Journal of Physical Chemistry C, vol. 115, no. 10, pp. 4174-4183, 2011.

[29] A. Popova, M. Christov, and A. Vasilev, "Inhibitive properties of quaternary ammonium bromides of $\mathrm{N}$-containing heterocycles on acid mild steel corrosion. part I: Gravimetric and voltammetric results," Corrosion Science, vol. 49, no. 8, pp. 3276-3289, 2007.

[30] S.-C. Chang, J.-M. Shieh, K.-C. Lin, B.-T. Dai, T.-C. Wang, C.-F. Chen, M.-S. Feng, Y.-H. Li, and C.-P. Lu, "Wetting effect on gap filling submicron damascene by an electrolyte free of levelers," Journal of Vacuum Science and Technology. B, Microelectronics and Nanometer Structures: Processing, Measurement, and Phenomena, vol. 20, no. 4, pp. 1311-1316, 2002.

[31] W.-P. Dow, M.-Y. Yen, W.-B. Lin, and S.-W. Ho, "Influence of molecular weight of polyethylene glycol on microvia filling by copper electroplating," Journal of The Electrochemical Society, vol. 152, no. 11, pp. C769-C775, 2005.

[32] D. Lu, A. Das, and W. Park, "Direct modeling of near field thermal radiation in a metamaterial," Optics Express, vol. 25, no. 11, pp. 12999-13009, 2017.

[33] Z. Thacker and P. J. Pinhero, "Terahertz spectroscopy of candidate oxides in mim diodes for terahertz detection," IEEE Transactions on Terahertz Science and Technology, vol. 6, no. 3, pp. 414-419, 2016.

[34] S.-K. Kim, D. Josell, and T. Moffat, "Electrodeposition of Cu in the PEI-PEGCl-SPS additive system reduction of overfill bump formation during superfilling," Journal of The Electrochemical Society, vol. 153, no. 9, pp. C616-C622, 2006. 
[35] P. M. Vereecken, R. A. Binstead, H. Deligianni, and P. C. Andricacos, "The chemistry of additives in damascene copper plating," IBM Journal of Research and Development, vol. 49, no. 1, p. 3, 2005.

[36] J. J. Kelly and A. C. West, "Copper deposition in the presence of polyethylene glycol I. quartz crystal microbalance study," Journal of The Electrochemical Society, vol. 145, no. 10, pp. 3472-3476, 1998.

[37] C. Chang, X. Lu, Z. Lei, Z. Wang, and C. Zhao, "2-mercaptopyridine as a new leveler for bottom-up filling of micro-vias in copper electroplating," Electrochimica Acta, vol. 208, pp. 33-38, 2016.

[38] M. J. Kim, Y. Seo, H. C. Kim, Y. Lee, S. Choe, Y. G. Kim, S. K. Cho, and J. J. Kim, "Galvanostatic bottom-up filling of TSV-like trenches: Choline-based leveler containing two quaternary ammoniums," Electrochimica Acta, vol. 163, pp. 174-181, 2015.

[39] J. J. Kelly, C. Tian, and A. C. West, "Leveling and microstructural effects of additives for copper electrodeposition," Journal of The Electrochemical Society, vol. 146, no. 7, pp. 2540-2545, 1999.

[40] Y.-B. Li, W. Wang, and Y.-L. Li, "Adsorption behavior and related mechanism of janus green b during copper via-filling process," Journal of The Electrochemical Society, vol. 156, no. 4, pp. D119-D124, 2009.

[41] S. Choe, M. J. Kim, H. C. Kim, S. K. Cho, S. H. Ahn, S.-K. Kim, and J. J. Kim, "Degradation of bis (3-sulfopropyl) disulfide and its influence on copper electrodeposition for feature filling," Journal of The Electrochemical Society, vol. 160, no. 12, pp. D3179-D3185, 2013.

[42] W. Li, H. Chung, C. Daeffler, J. A. Johnson, and R. H. Grubbs, "Application of ${ }^{1} \mathrm{H}$ DOSY for facile measurement of polymer molecular weights," Macromolecules, vol. 45, no. 24, pp. 9595-9603, 2012.

[43] Z. Nagy, J. Blaudeau, N. Hung, L. Curtiss, and D. Zurawski, "Chloride ion catalysis of the copper deposition reaction," Journal of The Electrochemical Society, vol. 142, no. 6, pp. L87-L89, 1995.

[44] C. Gabrielli, P. Moçotéguy, H. Perrot, and R. Wiart, "Mechanism of copper deposition in a sulphate bath containing chlorides," Journal of Electroanalytical Chemistry, vol. 572, no. 2, pp. 367-375, 2004.

[45] T. P. Moffat, D. Wheeler, and D. Josell, "Electrodeposition of copper in the SPS-PEG-Cl additive system i. kinetic measurements: Influence of SPS," Journal of The Electrochemical Society, vol. 151, no. 4, pp. C262-C271, 2004.

[46] P. Taephaisitphongse, Y. Cao, and A. C. West, "Electrochemical and fill studies of a multicomponent additive package for copper deposition," Journal of The Electrochemical Society, vol. 148, no. 7, pp. C492-C497, 2001. 
[47] W.-C. Tsai, C.-C. Wan, and Y.-Y. Wang, "Frequency effect of pulse plating on the uniformity of copper deposition in plated through holes," Journal of The Electrochemical Society, vol. 150, no. 5, pp. C267-C272, 2003.

[48] M. Fenn, G. Akuetey, and P. Donovan, "Electrical resistivity of Cu and Nb thin films," Journal of Physics: Condensed Matter, vol. 10, no. 8, p. 1707, 1998.

[49] E. Schmiedl, P. Wissmann, and H.-U. Finzel, "The electrical resistivity of ultrathin copper films," Z. Naturforsch. A, vol. 63, no. 10-11, pp. 739-744, 2008.

[50] A. Rashidi and A. Amadeh, "The effect of current density on the grain size of electrodeposited nanocrystalline nickel coatings," Surface and Coatings Technology, vol. 202, no. 16, pp. 3772-3776, 2008.

[51] "Copper deposition in a trench. COMSOL Multiphysics ${ }^{\circledR}$ v. 5.0. COMSOL AB, Stockholm, Sweden. 2015."

[52] "Superfilling electrodeposition. COMSOL Multiphysics ${ }^{\circledR}$ v. 5.0. COMSOL AB, Stockholm, Sweden. 2015."

[53] A. Frank and A. J. Bard, "The decomposition of the sulfonate additive sulfopropyl sulfonate in acid copper electroplating chemistries," Journal of The Electrochemical Society, vol. 150, no. 4, pp. C244-C250, 2003.

[54] W.-H. Lee, C.-C. Hung, S.-C. Chang, and Y.-L. Wang, "Bis-(3sodiumsulfopropyl disulfide) decomposition with cathodic current flowing in a copper-electroplating bath," Journal of The Electrochemical Society, vol. 157, no. 1, pp. H131-H135, 2010.

[55] E. B. Becker, G. F. Carey, and J. T. Oden, Finite Elements: Mathematical Aspects, vol. 4. Prentice Hall, 1983.

[56] R. R. Syms and J. R. Cozens, Optical Guided Waves and Devices. McGraw-Hill, 1992.

[57] Y. Fujishiro, "Taking advantage of S-parameter," TDK Corporation Application Center, 2000.

[58] M. Bass, E. W. Van Stryland, D. R. Williams, and W. L. Wolfe, Handbook of Optics, vol. 2. McGraw-Hill New York, 1995.

[59] M. A. Ordal, R. J. Bell, R. W. Alexander, L. L. Long, and M. R. Querry, "Optical properties of fourteen metals in the infrared and far infrared: Al, Co, $\mathrm{Cu}, \mathrm{Au}, \mathrm{Fe}, \mathrm{Pb}, \mathrm{Mo}, \mathrm{Ni}, \mathrm{Pd}, \mathrm{Pt}, \mathrm{Ag}, \mathrm{Ti}, \mathrm{V}$, and W," Applied Optics, vol. 24, no. 24, pp. 4493-4499, 1985.

[60] S. Arscott, F. Garet, P. Mounaix, L. Duvillaret, J.-L. Coutaz, and D. Lippens, "Terahertz time-domain spectroscopy of films fabricated from SU-8," Electronics Letters, vol. 35, no. 3, pp. 243-244, 1999. 
[61] T. Pompe, A. Fery, S. Herminghaus, A. Kriele, H. Lorenz, and J. Kotthaus, "Submicron contact printing on silicon using stamp pads," Langmuir, vol. 15, no. 7, pp. 2398-2401, 1999.

[62] C. Täubert, D. Kolb, U. Memmert, and H. Meyer, "Adsorption of the additives MPA, MPSA, and SPS onto $\mathrm{Cu}$ (111) from sulfuric acid solutions," Journal of The Electrochemical Society, vol. 154, no. 6, pp. D293-D299, 2007.

[63] A. C. West, "Theory of filling of high-aspect ratio trenches and vias in presence of additives," Journal of The Electrochemical Society, vol. 147, no. 1, pp. 227$232,2000$.

[64] W.-P. Dow, H.-S. Huang, M.-Y. Yen, and H.-H. Chen, "Roles of chloride ion in microvia filling by copper electrodeposition II. studies using epr and galvanostatic measurements," Journal of The Electrochemical Society, vol. 152, no. 2, pp. C77-C88, 2005.

[65] J. M. Halpern, R. Urbanski, A. K. Weinstock, D. F. Iwig, R. T. Mathers, and H. A. Von Recum, "A biodegradable thermoset polymer made by esterification of citric acid and glycerol," Journal of Biomedical Materials Research Part A, vol. 102, no. 5, pp. 1467-1477, 2014.

[66] F. Fan, W. Wang, A. P. Holt, H. Feng, D. Uhrig, X. Lu, T. Hong, Y. Wang, N.-G. Kang, J. Mays, et al., "Effect of molecular weight on the ion transport mechanism in polymerized ionic liquids," Macromolecules, vol. 49, no. 12, pp. 4557-4570, 2016.

[67] S. Zhang, "Fabrication of novel biomaterials through molecular self-assembly," Nature Biotechnology, vol. 21, no. 10, p. 1171, 2003.

[68] T. Pakalns, K. L. Haverstick, G. B. Fields, J. B. McCarthy, D. L. Mooradian, and M. Tirrell, "Cellular recognition of synthetic peptide amphiphiles in selfassembled monolayer films," Biomaterials, vol. 20, no. 23-24, pp. 2265-2279, 1999.

[69] J. D. Hartgerink, E. Beniash, and S. I. Stupp, "Self-assembly and mineralization of peptide-amphiphile nanofibers," Science, vol. 294, no. 5547, pp. 1684-1688, 2001.

[70] S. Eskandari, T. Guerin, I. Toth, and R. J. Stephenson, "Recent advances in self-assembled peptides: Implications for targeted drug delivery and vaccine engineering," Advanced Drug Delivery Reviews, vol. 110, pp. 169-187, 2017.

[71] A. Dehsorkhi, R. M. Gouveia, A. M. Smith, I. W. Hamley, V. Castelletto, C. J. Connon, M. Reza, and J. Ruokolainen, "Self-assembly of a dual functional bioactive peptide amphiphile incorporating both matrix metalloprotease substrate and cell adhesion motifs," Soft Matter, vol. 11, no. 16, pp. 3115-3124, 2015 . 
[72] M. A. Biesalski, A. Knaebel, R. Tu, and M. Tirrell, "Cell adhesion on a polymerized peptide-amphiphile monolayer," Biomaterials, vol. 27, no. 8, pp. 1259 1269, 2006.

[73] J. Marek, P. Stodulka, J. Cabal, O. Soukup, M. Pohanka, J. Korabecny, K. Musilek, and K. Kuca, "Preparation of the pyridinium salts differing in the length of the N-alkyl substituent," Molecules, vol. 15, no. 3, pp. 1967-1972, 2010 .

[74] B. Kamm, M. Kamm, A. Kiener, and H.-P. Meyer, "Polycarnitinea new biomaterial," Applied Microbiology and Biotechnology, vol. 67, no. 1, pp. 1-7, 2005.

[75] B. D. Ulery, Y. Phanse, A. Sinha, M. J. Wannemuehler, B. Narasimhan, and B. H. Bellaire, "Polymer chemistry influences monocytic uptake of polyanhydride nanospheres," Pharmaceutical Research, vol. 26, no. 3, pp. 683-690, 2009.

[76] B. D. Ulery, L. S. Nair, and C. T. Laurencin, "Biomedical applications of biodegradable polymers," Journal of Polymer Science Part B: Polymer Physics, vol. 49, no. 12, pp. 832-864, 2011.

[77] B. D. Ulery, D. Kumar, A. E. Ramer-Tait, D. W. Metzger, M. J. Wannemuehler, and B. Narasimhan, "Design of a protective single-dose intranasal nanoparticlebased vaccine platform for respiratory infectious diseases," PlOS One, vol. 6, no. 3, p. e17642, 2011.

[78] K. W.-H. Lo, B. D. Ulery, K. M. Ashe, and C. T. Laurencin, "Studies of bone morphogenetic protein-based surgical repair," Advanced Drug Delivery Reviews, vol. 64, no. 12, pp. 1277-1291, 2012.

[79] H. Usui, N. Uchida, and H. Sakaguchi, "Influence of order in stepwise electroless deposition on anode properties of thick-film electrodes consisting of Si particles coated with Ni and Cu," Journal of Power Sources, vol. 196, no. 23, pp. 1024410248, 2011.

[80] M. Thitsa, Y. Song, and S. Albin, "Effect of oxidation, etching, and thin-film deposition on silicon photonic crystals," Journal of the Electrochemical Society, vol. 155, no. 6, pp. H351-H356, 2008.

[81] E. Norkus, A. Vaškelis, J. Jačiauskiene, J. VaičIuniene, E. Gaidamauskas, and D. Macalady, "Environmentally friendly natural polyhydroxylic compounds in electroless copper plating baths: application of xylitol, $D$-mannitol and $D$ sorbitol as copper (II) ligands," Journal of Applied Electrochemistry, vol. 35, no. 1, pp. 41-47, 2005.

[82] A. Hung and K.-M. Chen, "Mechanism of hypophosphite-reduced electroless copper plating," Journal of the Electrochemical Society, vol. 136, no. 1, pp. 7275, 1989 . 
[83] P. C. Hidber, P. F. Nealey, W. Helbig, and G. M. Whitesides, "New strategy for controlling the size and shape of metallic features formed by electroless deposition of copper: Microcontact printing of catalysts on oriented polymers, followed by thermal shrinkage," Langmuir, vol. 12, no. 21, pp. 5209-5215, 1996.

[84] X. Han, W. Wu, Y. Li, Z. Li, Y. Hao, and G. Yan, "Electroless copper plating applicable for bulk-silicon micromachined radio frequency inductor," Thin Solid Films, vol. 515, no. 4, pp. 2607-2611, 2006.

[85] E. G. Geterud, P. Bergmark, and J. Yang, "Lightweight waveguide and antenna components using plating on plastics," in Antennas and Propagation (EuCAP), 2013 7th European Conference on, pp. 1812-1815, IEEE, 2013.

[86] X. Gan, Y. Wu, L. Liu, and W. Hu, "Effects of $\mathrm{K}_{4} \mathrm{Fe}(\mathrm{CN})_{6}$ on electroless copper plating using hypophosphite as reducing agent," Journal of Applied Electrochemistry, vol. 37, no. 8, pp. 899-904, 2007.

[87] X. Cui, D. A. Hutt, and P. P. Conway, "Investigation of the adhesion of electroless copper to glass substrates," MRS Online Proceedings Library Archive, vol. 1113, 2008.

[88] R. C. Agarwala, "Studies on electroless coatings," in Defect and Diffusion Forum, vol. 237, pp. 952-957, Trans Tech Publications, 2005.

[89] C. A. Schneider, W. S. Rasband, and K. W. Eliceiri, "NIH Image to ImageJ: 25 years of image analysis," Nature Methods, vol. 9, no. 7, p. 671, 2012.

[90] D. Nečas and P. Klapetek, "Gwyddion: an open-source software for spm data analysis," Open Physics, vol. 10, no. 1, pp. 181-188, 2012.

[91] D. Wheeler, D. Josell, and T. P. Moffat, "Modeling superconformal electrodeposition using the level set method," Journal of The Electrochemical Society, vol. 150, no. 5, pp. C302-C310, 2003.

[92] W. Luo, J. Zhang, Z. Chen, Y. Zhu, L. Gao, and M. Li, "Modeling the copper electrodeposition of through-silicon-vias corresponded to linear sweep voltammetry," Electrochemistry, vol. 84, no. 7, pp. 516-522, 2016.

[93] M. Georgiadou, D. Veyret, R. Sani, and R. Alkire, "Simulation of shape evolution during electrodeposition of copper in the presence of additive," Journal of The Electrochemical Society, vol. 148, no. 1, pp. C54-C58, 2001.

[94] R. B. Bird, "Transport phenomena," Applied Mechanics Reviews, vol. 55, no. 1, pp. R1-R4, 2002.

[95] D. Josell, D. Wheeler, C. Witt, and T. P. Moffat, "Seedless superfill: copper electrodeposition in trenches with ruthenium barriers," Electrochemical and Solid-State Letters, vol. 6, no. 10, pp. C143-C145, 2003. 
[96] D. M. Mattox and V. Mattox, Vacuum Coating Technology. Springer, 2003.

[97] J. E. Mahan, "Physical vapor deposition of thin films," Physical Vapor Deposition of Thin Films, p. 336, 2000.

[98] S. C. Yu, S. Hou, and W. K. Chan, "Synthesis and properties of polyamides and polyesters on the basis of 2,2-bipyridine-5,5-dicarboxylic acid and the corresponding polymer-ruthenium complexes," Macromolecules, vol. 33, no. 9, pp. 3259-3273, 2000.

[99] P. Ponath, A. B. Posadas, R. C. Hatch, and A. A. Demkov, "Preparation of a clean Ge (001) surface using oxygen plasma cleaning," Journal of Vacuum Science and Technology B, Nanotechnology and Microelectronics: Materials, Processing, Measurement, and Phenomena, vol. 31, no. 3, p. 031201, 2013.

[100] A. Danilov, E. Molodkina, and Y. M. Polukarov, "Initial stages of copper electrocrystallization on a glassy-carbon ring-disk electrode from sulfate electrolytes of various acidity: A cyclic voltammetry study," Russian Journal of Electrochemistry, vol. 38, no. 7, pp. 732-742, 2002.

[101] J. D. Adolf, Function of Additives in Copper Electrodeposition for Semiconductor Device Metallization. PhD thesis, Case Western Reserve University, 2008.

[102] Y. S. Won, D. Cho, Y. Kim, J. Lee, and S. S. Park, "Degradation of poly (ethylene glycol) by electrolysis during the $\mathrm{Cu}$ electroplating: A combined experimental and density functional theory study," Journal of Applied Polymer Science, vol. 117, no. 4, pp. 2083-2089, 2010.

[103] L. DUrzo, H. Wang, C. Tang, A. Pa, and C. Zhi, "Solid phase extractionhigh-performance liquid chromatography detection of organic additives in acidic copper baths II. polyethylene-glycol based suppressors," Journal of The Electrochemical Society, vol. 152, no. 10, pp. C697-C700, 2005.

[104] H. E. Gottlieb, V. Kotlyar, and A. Nudelman, "NMR chemical shifts of common laboratory solvents as trace impurities," The Journal of Organic Chemistry, vol. 62 , no. 21 , pp. $7512-7515,1997$.

[105] E. Garcia-Cardona, E. H. Wong, and D. P. Barkey, "NMR spectral studies of interactions between the accelerants SPS and MPS and copper chlorides," Journal of the Electrochemical Society, vol. 158, no. 3, pp. D143-D148, 2011.

[106] D. K. Kotter, S. D. Novack, W. D. Slafer, and P. Pinhero, "Solar nantenna electromagnetic collectors," in ASME 2008 2nd International Conference on Energy Sustainability collocated with the Heat Transfer, Fluids Engineering, and 3rd Energy Nanotechnology Conferences, pp. 409-415, American Society of Mechanical Engineers, 2008. 
[107] K. G. Schmitt, R. Schmidt, H. F. von Horsten, G. Vazhenin, and A. A. Gewirth, "3-mercapto-1-propanesulfonate for $\mathrm{Cu}$ electrodeposition studied by in situ shell-isolated nanoparticle-enhanced raman spectroscopy, density functional theory calculations, and cyclic voltammetry," The Journal of Physical Chemistry C, vol. 119, no. 41, pp. 23453-23462, 2015.

[108] Y. Wang, T. Sun, T. Paudel, Y. Zhang, Z. Ren, and K. Kempa, "Metamaterialplasmonic absorber structure for high efficiency amorphous silicon solar cells," Nano Letters, vol. 12, no. 1, pp. 440-445, 2011.

[109] C. Simovski, "Material parameters of metamaterials (a review)," Optics and Spectroscopy, vol. 107, no. 5, p. 726, 2009.

[110] R. A. Shelby, D. R. Smith, and S. Schultz, "Experimental verification of a negative index of refraction," Science, vol. 292, no. 5514, pp. 77-79, 2001.

[111] S. A. Ramakrishna, "Physics of negative refractive index materials," Reports on Progress in Physics, vol. 68, no. 2, p. 449, 2005.

[112] A. Oliva, R. Maldonado, E. Díaz, and A. Montalvo, "A high absorbance material for solar collectors' applications," in IOP Conference Series: Materials Science and Engineering, vol. 45, p. 012019, IOP Publishing, 2013.

[113] F. Miyamaru, S. Kuboda, K. Taima, K. Takano, M. Hangyo, and M. W. Takeda, "Three-dimensional bulk metamaterials operating in the terahertz range," $A p$ plied Physics Letters, vol. 96, no. 8, p. 081105, 2010.

[114] N. Landy, S. Sajuyigbe, J. J. Mock, D. R. Smith, and W. J. Padilla, "Perfect metamaterial absorber," Physical Review Letters, vol. 100, no. 20, p. 207402, 2008 .

[115] C. Wagner, W. M. Kaiser, J. Mulkens, and D. G. Flagello, "Advanced technology for extending optical lithography," in Optical Microlithography XIII, vol. 4000, pp. 344-358, International Society for Optics and Photonics, 2000.

[116] C. Wang, M. An, P. Yang, and J. Zhang, "Prediction of a new leveler (n-butylmethyl piperidinium bromide) for through-hole electroplating using molecular dynamics simulations," Electrochemistry Communications, vol. 18, pp. 104-107, 2012 .

[117] J. Reid, "Copper electrodeposition: principles and recent progress," Japanese Journal of Applied Physics, vol. 40, no. 4S, p. 2650, 2001.

[118] S. Miura and H. Honma, "Advanced copper electroplating for application of electronics," Surface and Coatings Technology, vol. 169, pp. 91-95, 2003.

[119] C. Meudre, L. Ricq, J.-Y. Hihn, V. Moutarlier, A. Monnin, and O. Heintz, "Adsorption of gelatin during electrodeposition of copper and tin-copper alloys from acid sulfate electrolyte," Surface and Coatings Technology, vol. 252, pp. 93-101, 2014. 
[120] O. Lühn, C. Van Hoof, W. Ruythooren, and J.-P. Celis, "Filling of microvia with an aspect ratio of 5 by copper electrodeposition," Electrochimica Acta, vol. 54, no. 9, pp. 2504-2508, 2009.

[121] S.-K. Kim, S. Hwang, S. K. Cho, and J. J. Kim, "Leveling of superfilled damascene Cu film using two-step electrodeposition," Electrochemical and Solid-State Letters, vol. 9, no. 2, pp. C25-C28, 2006.

[122] M. Ibrahim and R. Bakdash, "New non-cyanide acidic copper electroplating bath based on glutamate complexing agent," Surface and Coatings Technology, vol. 282, pp. 139-148, 2015.

[123] W.-P. Dow, H.-S. Huang, and Z. Lin, "Interactions between brightener and chloride ions on copper electroplating for laser-drilled via-hole filling," Electrochemical and Solid-State Letters, vol. 6, no. 9, pp. C134-C136, 2003.

[124] S.-Y. Chiu, J.-M. Shieh, S.-C. Chang, K.-C. Lin, B.-T. Dai, C.-F. Chen, and M.S. Feng, "Characterization of additive systems for damascene $\mathrm{Cu}$ electroplating by the superfilling profile monitor," Journal of Vacuum Science and Technology B: Microelectronics and Nanometer Structures Processing, Measurement, and Phenomena, vol. 18, no. 6, pp. 2835-2841, 2000.

[125] R. Beica, C. Sharbono, and T. Ritzdorf, "Through silicon via copper electrodeposition for 3D integration," in Electronic Components and Technology Conference, 2008. ECTC 2008. 58th, pp. 577-583, IEEE, 2008.

[126] A. Damjanovic, T. H. Setty, and J. Bockris, "Effect of crystal plane on the mechanism and the kinetics of copper electrocrystallization," Journal of The Electrochemical Society, vol. 113, no. 5, pp. 429-440, 1966.

[127] C. Wang, J. Zhang, P. Yang, and M. An, "Electrochemical behaviors of Janus Green B in through-hole copper electroplating: An insight by experiment and density functional theory calculation using Safranine T as a comparison," Electrochimica Acta, vol. 92, pp. 356-364, 2013.

[128] M. A. Plante, S. Fairlie, B. Bailey, and I. N. Acworth, "Measurement and control of copper additives in electroplating baths by high-performance liquid chromatography,"

[129] J. J. Kellya and A. C. West, "Leveling of $200 \mathrm{~nm}$ features by organic additives," Electrochemical and Solid-State Letters, vol. 2, no. 11, pp. 561-563, 1999.

[130] W.-P. Dow, C.-C. Li, M.-W. Lin, G.-W. Su, and C.-C. Huang, "Copper fill of microvia using a thiol-modified $\mathrm{Cu}$ seed layer and various levelers," Journal of the Electrochemical Society, vol. 156, no. 8, pp. D314-D320, 2009.

[131] Y. Gu, J. Liu, S. Qu, Y. Deng, X. Han, W. Hu, and C. Zhong, "Electrodeposition of alloys and compounds from high-temperature molten salts," Journal of Alloys and Compounds, vol. 690, pp. 228-238, 2017. 
[132] M. Armand, F. Endres, D. R. MacFarlane, H. Ohno, and B. Scrosati, "Ionicliquid materials for the electrochemical challenges of the future," in Materials For Sustainable Energy: A Collection of Peer-Reviewed Research and Review Articles from Nature Publishing Group, pp. 129-137, World Scientific, 2011.

[133] M. T. Reetz and W. Helbig, "Size-selective synthesis of nanostructured transition metal clusters," Journal of the American Chemical Society, vol. 116, no. 16, pp. 7401-7402, 1994.

[134] A. Rashidi and A. Amadeh, "Effect of electroplating parameters on microstructure of nanocrystalline nickel coatings," Journal of Materials Science and Technology, vol. 26, no. 1, pp. 82-86, 2010.

[135] C.-T. Lin and K.-L. Lin, "Effects of current density and deposition time on electrical resistivity of electroplated Cu layers," Journal of Materials Science: Materials in Electronics, vol. 15, no. 11, pp. 757-762, 2004.

[136] M. Kuwabara and H. Shimooka, "Grain size dependence of the critical current density in $\mathrm{YBa}_{2} \mathrm{Cu}_{3} \mathrm{O}_{x}$ superconductors," Applied Physics Letters, vol. 55, no. 26, pp. 2781-2783, 1989.

[137] N. Ibl and K. Schadegg, "Surface roughness effects in the electrodeposition of copper in the limiting current range," Journal of the Electrochemical Society, vol. 114, no. 1, pp. 54-58, 1967.

[138] D. P. Barkey, R. H. Muller, and C. W. Tobias, "Roughness development in metal electrodeposition II. Stability theory," Journal of the Electrochemical Society, vol. 136, no. 8, pp. 2207-2214, 1989.

[139] G. Lee, J. Kim, K. Kim, and J. W. Han, "Precise control of defects in graphene using oxygen plasma," Journal of Vacuum Science and Technology A: Vacuum, Surfaces, and Films, vol. 33, no. 6, p. 060602, 2015.

[140] C. Lambare, P.-Y. Tessier, F. Poncin-Epaillard, and D. Debarnot, "Plasma functionalization and etching for enhancing metal adhesion onto polymeric substrates," RSC Advances, vol. 5, no. 77, pp. 62348-62357, 2015.

[141] C. Kemmerer and R. Mills, "Adhesion of thin films of evaporated titaniumcopper after electroplating," Journal of Vacuum Science and Technology, vol. 16, no. 2, pp. 352-355, 1979.

[142] L. Brandt, Z. Liu, H. Fu, S. Hunegnaw, and T. Magaya, "Adhesive enabling technology for directly plating copper onto glass," Additional Papers and Presentations, vol. 2014, no. DPC, pp. 001913-001936, 2014.

[143] S. Choe, M. J. Kim, K. H. Kim, H. C. Kim, J. C. Song, S.-K. Kim, and J. J. Kim, "Accuracy improvement in cyclic voltammetry stripping analysis of thiourea concentration in copper plating baths," Journal of The Electrochemical Society, vol. 162, no. 4, pp. H294-H300, 2015. 
[144] J. Y. Lee and T.-C. Tan, "Cyclic voltammetry of electrodeposition of metal on electrosynthesized polypyrrole film," Journal of The Electrochemical Society, vol. 137, no. 5, pp. 1402-1408, 1990.

[145] F. Ebrahimi and Z. Ahmed, "The effect of current density on properties of electrodeposited nanocrystalline nickel," Journal of Applied Electrochemistry, vol. 33, no. 8, pp. 733-739, 2003.

[146] A. He, T. Osborn, S. A. B. Allen, and P. A. Kohl, "Low-temperature bonding of copper pillars for all-copper chip-to-substrate interconnections," Electrochemical and Solid-State Letters, vol. 9, no. 12, pp. C192-C195, 2006.

[147] X. Fu, H. Chai, and X. Li, "Silicon nanoporous pillar array and its surface copper deposition," Chinese Science Bulletin, vol. 50, no. 14, pp. 1424-1428, 2005 .

[148] W.-P. Dow, C.-W. Lu, J.-Y. Lin, and F.-C. Hsu, "Highly selective Cu electrodeposition for filling through silicon holes," Electrochemical and Solid-State Letters, vol. 14, no. 6, pp. D63-D67, 2011.

[149] P. Dixit, J. Miao, and R. Preisser, "Fabrication of high aspect ratio $35 \mu \mathrm{m}$ pitch through-wafer copper interconnects by electroplating for 3-D wafer stacking," Electrochemical and Solid-State Letters, vol. 9, no. 10, pp. G305-G308, 2006.

[150] R. Tenno and A. Pohjoranta, "An ale model for prediction and control of the microvia fill process with two additives," Journal of The Electrochemical Society, vol. 155, no. 5, pp. D383-D388, 2008.

[151] E. Rusli, F. Xue, T. O. Drews, P. M. Vereecken, P. Andricacos, H. Deligianni, R. D. Braatz, and R. C. Alkire, "Effect of additives on shape evolution during electrodeposition II. Parameter estimation from roughness evolution experiments," Journal of The Electrochemical Society, vol. 154, no. 11, pp. D584D597, 2007.

[152] Y. Qin, X. Li, F. Xue, P. M. Vereecken, P. Andricacos, H. Deligianni, R. D. Braatz, and R. C. Alkire, "Effect of additives on shape evolution during electrodeposition III. trench infill for on-chip interconnects," Journal of The Electrochemical Society, vol. 155, no. 3, pp. D223-D233, 2008.

[153] T. P. Moffat, D. Wheeler, M. D. Edelstein, and D. Josell, "Superconformal film growth: Mechanism and quantification," IBM Journal of Research and Development, vol. 49, no. 1, pp. 19-36, 2005.

[154] X. Li, T. O. Drews, E. Rusli, F. Xue, Y. He, R. Braatz, and R. Alkire, "Effect of additives on shape evolution during electrodeposition I. Multiscale simulation with dynamically coupled kinetic monte carlo and moving-boundry finite-volume codes," Journal of The Electrochemical Society, vol. 154, no. 4, pp. D230-D240, 2007. 
[155] F. Caspers, "RF engineering basic concepts: S-parameters," CERN Yellow Report, 2012.

[156] W. L. Yan and R. Bai, "Adsorption of lead and humic acid on chitosan hydrogel beads," Water Research, vol. 39, no. 4, pp. 688-698, 2005.

[157] J. Vivek and I. J. Burgess, "Quaternary ammonium bromide surfactant adsorption on low-index surfaces of gold. 1. Au (111)," Langmuir, vol. 28, no. 11, pp. 5031-5039, 2012.

[158] T. J. Rockey, M. Yang, and H.-L. Dai, "Aniline on ag (1 1 1): Adsorption configuration, adsorbate-substrate bond, and inter-adsorbate interactions," Surface Science, vol. 589, no. 1-3, pp. 42-51, 2005.

[159] L. Raehm, J.-M. Kern, J.-P. Sauvage, C. Hamann, S. Palacin, and J.-P. Bourgoin, "Disulfide-and thiol-incorporating copper catenanes: Synthesis, deposition onto gold, and surface studies," Chemistry-A European Journal, vol. 8, no. 9, pp. 2153-2162, 2002.

[160] M. Preuss, W. Schmidt, and F. Bechstedt, "Coulombic amino group-metal bonding: Adsorption of adenine on Cu (110)," Physical Review Letters, vol. 94, no. 23, p. 236102, 2005.

[161] P. E. Laibinis, G. M. Whitesides, D. L. Allara, Y. T. Tao, A. N. Parikh, and R. G. Nuzzo, "Comparison of the structures and wetting properties of selfassembled monolayers of $N$-alkanethiols on the coinage metal surfaces, copper, silver, and gold," Journal of the American Chemical Society, vol. 113, no. 19, pp. 7152-7167, 1991.

[162] P. E. Laibinis and G. M. Whitesides, " $\omega$-terminated alkanethiolate monolayers on surfaces of copper, silver, and gold have similar wettabilities," Journal of the American Chemical Society, vol. 114, no. 6, pp. 1990-1995, 1992.

[163] P. E. Laibinis and G. M. Whitesides, "Self-assembled monolayers of nalkanethiolates on copper are barrier films that protect the metal against oxidation by air," Journal of the American Chemical Society, vol. 114, no. 23, pp. 9022-9028, 1992.

[164] H. Keller, P. Simak, W. Schrepp, and J. Dembowski, "Surface chemistry of thiols on copper: an efficient way of producing multilayers," Thin Solid Films, vol. 244, no. 1-2, pp. 799-805, 1994.

[165] R. C. Hoft, M. J. Ford, A. M. McDonagh, and M. B. Cortie, "Adsorption of amine compounds on the $\mathrm{Au}$ (111) surface: a density functional study," The Journal of Physical Chemistry C, vol. 111, no. 37, pp. 13886-13891, 2007.

[166] Y. J. Han, X. Zhang, and G. W. Leach, "Shape control of electrodeposited copper films and nanostructures through additive effects," Langmuir, vol. 30, no. 12, pp. 3589-3598, 2014. 
[167] M. El-Batouti, S. H. Salaam, A.-M. M. Ahmed, and H. EL-SHAMY, "Effect of some organic compounds on electrodeposition of copper metal in copper cathode," Asian Journal of Chemistry, vol. 28, no. 5, 2016.

[168] V. Di Castro, M. Beccari, F. Bruni, F. Caprioli, and F. Decker, "Comparison of the protective effect of aromatic thiols adsorbed on copper," Surface and Interface Analysis, vol. 42, no. 6-7, pp. 601-604, 2010.

[169] F. Caprioli, F. Decker, A. G. Marrani, M. Beccari, and V. Di Castro, "Copper protection by self-assembled monolayers of aromatic thiols in alkaline solutions," Physical Chemistry Chemical Physics, vol. 12, no. 32, pp. 9230-9238, 2010.

[170] J. Benard, "The chemical adsorption of sulfur on metals: Thermodynamics and structure," Catalysis Reviews, vol. 3, no. 1, pp. 93-109, 1970.

[171] T. Baunach, V. Ivanova, D. A. Scherson, and D. M. Kolb, "Self-assembled monolayers of 4-mercaptopyridine on $\mathrm{Au}$ (111): A potential-induced phase transition in sulfuric acid solutions," Langmuir, vol. 20, no. 7, pp. 2797-2802, 2004.

[172] J. Valentine, S. Zhang, T. Zentgraf, E. Ulin-Avila, D. A. Genov, G. Bartal, and $\mathrm{X}$. Zhang, "Three-dimensional optical metamaterial with a negative refractive index," Nature, vol. 455, no. 7211, p. 376, 2008.

[173] C. M. Soukoulis and M. Wegener, "Past achievements and future challenges in the development of three-dimensional photonic metamaterials," Nature Photonics, vol. 5, no. 9, p. 523, 2011.

[174] I. Smolyaninov, Y. Hung, and C. Davis, "Two-dimensional metamaterial structure exhibiting reduced visibility at $500 \mathrm{~nm}, "$ Optics Letters, vol. 33, no. 12, pp. 1342-1344, 2008.

[175] D. Schurig, J. Mock, B. Justice, S. A. Cummer, J. B. Pendry, A. Starr, and D. Smith, "Metamaterial electromagnetic cloak at microwave frequencies," Science, vol. 314, no. 5801, pp. 977-980, 2006.

[176] N. Liu, H. Guo, L. Fu, S. Kaiser, H. Schweizer, and H. Giessen, "Threedimensional photonic metamaterials at optical frequencies," Nature Materials, vol. 7 , no. 1, p. 31, 2008 .

[177] C. L. Holloway, E. F. Kuester, J. A. Gordon, J. O'Hara, J. Booth, and D. R. Smith, "An overview of the theory and applications of metasurfaces: The twodimensional equivalents of metamaterials," IEEE Antennas and Propagation Magazine, vol. 54, no. 2, pp. 10-35, 2012.

[178] J. K. Gansel, M. Thiel, M. S. Rill, M. Decker, K. Bade, V. Saile, G. von Freymann, S. Linden, and M. Wegener, "Gold helix photonic metamaterial as broadband circular polarizer," Science, vol. 325, no. 5947, pp. 1513-1515, 2009. 
[179] T. Ueda, N. Michishita, A. Lai, M. Akiyama, and T. Itoh, "2.5-D stacked composite right/left-handed metamaterial structures using dielectric resonators and parallel mesh plates," in Microwave Symposium Digest, 2008 IEEE MTT-S International, pp. 335-338, IEEE, 2008.

[180] R. Shelby, D. Smith, S. Nemat-Nasser, and S. Schultz, "Microwave transmission through a two-dimensional, isotropic, left-handed metamaterial," Applied Physics Letters, vol. 78, no. 4, pp. 489-491, 2001.

[181] J. Parrón, G. Junkin, and J. M. Rius, "Improving the performance of the multilevel matrix decomposition algorithm for 2.5-D structures. application to metamaterials," in Antennas and Propagation Society International Symposium 2006, IEEE, pp. 2941-2944, IEEE, 2006.

[182] C. Helgert, C. Rockstuhl, C. Etrich, C. Menzel, E.-B. Kley, A. Tünnermann, F. Lederer, and T. Pertsch, "Effective properties of amorphous metamaterials," Physical Review B, vol. 79, no. 23, p. 233107, 2009.

[183] S. Zhang, Y.-S. Park, J. Li, X. Lu, W. Zhang, and X. Zhang, "Negative refractive index in chiral metamaterials," Physical Review Letters, vol. 102, no. 2, p. 023901, 2009.

[184] D. Wu, N. Fang, C. Sun, X. Zhang, W. J. Padilla, D. N. Basov, D. R. Smith, and S. Schultz, "Terahertz plasmonic high pass filter," Applied Physics Letters, vol. 83, no. 1, pp. 201-203, 2003.

[185] M. Ortolani, J. Lee, U. Schade, and H.-W. Hübers, "Surface roughness effects on the terahertz reflectance of pure explosive materials," Applied Physics Letters, vol. 93, no. 8, p. 081906, 2008.

[186] S. Nashima, O. Morikawa, K. Takata, and M. Hangyo, "Measurement of optical properties of highly doped silicon by terahertz time domain reflection spectroscopy," Applied Physics Letters, vol. 79, no. 24, pp. 3923-3925, 2001.

[187] N. Landy, C. Bingham, T. Tyler, N. Jokerst, D. Smith, and W. Padilla, "Design, theory, and measurement of a polarization-insensitive absorber for terahertz imaging," Physical Review B, vol. 79, no. 12, p. 125104, 2009.

[188] T.-I. Jeon and D. Grischkowsky, "Characterization of optically dense, doped semiconductors by reflection $\mathrm{THz}$ time domain spectroscopy," Applied Physics Letters, vol. 72, no. 23, pp. 3032-3034, 1998.

[189] S. Howells and L. Schlie, "Transient terahertz reflection spectroscopy of undoped InSb from 0.1 to 1.1 THz," Applied Physics Letters, vol. 69, no. 4, pp. 550-552, 1996.

[190] D. K. Kotter, S. D. Novack, W. Slafer, and P. Pinhero, "Theory and manufacturing processes of solar nanoantenna electromagnetic collectors," Journal of Solar Energy Engineering, vol. 132, no. 1, p. 011014, 2010. 
[191] L. Cheng, T. Zhou, and S. Kuang, "Discussion more about copper-phosphorus anode in bright sulfate copper plating process," Electroplating Finishing, vol. 2, p. 005, 1999.

[192] H.-T. Chen, W. J. Padilla, J. M. Zide, A. C. Gossard, A. J. Taylor, and R. D. Averitt, "Active terahertz metamaterial devices," Nature, vol. 444, no. 7119, pp. 597-600, 2006. 


\section{VITA}

Shendu Yang was born on February 28, 1989 in China. She received her B.S. degree at Beijing University of Chemical Technology in July 2012 and M.S. degree at the University of Missouri in July 2014. She has worked as a research assistant under Dr. Patrick Pinhero in the field of electrochemistry and semiconductor since January 2013. She is currently working on three-dimensional metamaterial fabrication and FEM simulation for industrial waste heat harvesting. 